FT-2004-02

\title{
Low Energy Tests of the Weak Interaction
}

\author{
J. Erler ${ }^{1}$ and M.J. Ramsey-Musolf ${ }^{2,3}$ \\ ${ }^{1}$ Instituto de Física, Universidad Nacional Autónoma de México, México \\ ${ }^{2}$ Kellogg Radiation Laboratory, California Institute of Technology, USA \\ ${ }^{3}$ Department of Physics, University of Connecticut, USA
}

October 23, 2018

\begin{abstract}
The study of low energy weak interactions of light quarks and leptons continues to provide important insights into both the Standard Model as well as the physics that may lie beyond it. We review the status and future prospects for low energy electroweak physics. Recent important experimental and theoretical developments are discussed and open theoretical issues are highlighted. Particular attention is paid to neutrino physics, searches for permanent electric dipole moments, neutral current tests of the running of the weak mixing angle, weak decays, and muon physics. We argue that the broad range of such studies provides an important complement to high energy collider searches for physics beyond the Standard Model. The use of low energy weak interactions to probe novel aspects of hadron structure is also discussed.
\end{abstract}




\section{Contents}

$1 \quad$ Introduction 3

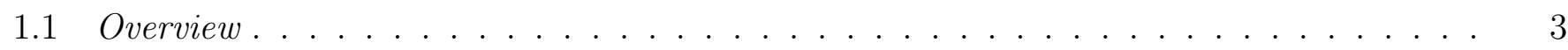

1.2 The Standard Mode . . . . . . . . . . . . . . . . . . . . . . . . 4

1.3 Status of the Standard Mode . . . . . . . . . . . . . . . . . . . . . . . . 7

1.4 Physics Beyond the Standard Mode . . . . . . . . . . . . . . . . . . . . . . 11

2 Weak Neutral Current Phenomena 13

2.1 Neutrino Scatterind . . . . . . . . . . . . . . . . . . . . . . . . . . . . 13

2.2 Charaed Lepton Scatterind . . . . . . . . . . . . . . . . . . . . . . . . 16

2.3 Parity Violation in Atoms . . . . . . . . . . . . . . . . . . . . . . . 19

3 Charged Current Phenomena 22

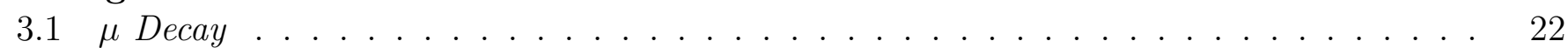

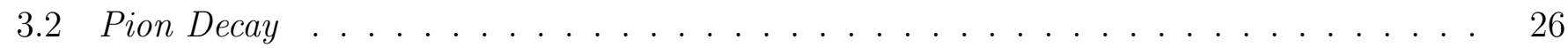

3.3 Beta Decay. Kaon Decay. and CKM Unitaritz . . . . . . . . . . . . . . . . . 29

4 Rare and Forbidden Processes 33

4.1 Electric Dipole Moments . . . . . . . . . . . . . . . . . . . . . . . . 33

4.1 .1 Experimental developments $\ldots \ldots \ldots \ldots \ldots \ldots$

4.1 .2 Theoretical implications . . . . . . . . . . . . . . . . . . 38

4.1 .3 EDMs and the barvon asvmmetry of the Universe . . . . . . . . . . . . 42

4.2 Lepton Flavor Violation $\ldots \ldots \ldots \ldots \ldots$

5 Lepton Properties 45

5.1 Neutrino masses and mixind . . . . . . . . . . . . . . . . . . 45

5.2 Solar Neutrinos . . . . . . . . . . . . . . . . . . . . . . . . . 47

5.2 .1 Rates . . . . . . . . . . . . . . . . . . . . 48

5.2 .2 Time variations and spectral distortions . . . . . . . . . . . . . . 50

5.2 .3 The large mixing angle MSW solution to the solar neutrino problem . . . . . . . 51

5.3 Atmospheric Neutrinos . . . . . . . . . . . . . . . . . . . . . . . 53

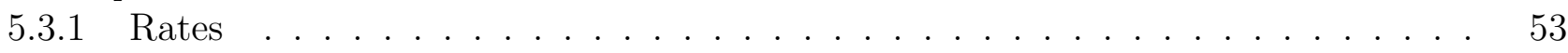

5.3 .2 Zenith angle and spectral distributions . . . . . . . . . . . . . . 53

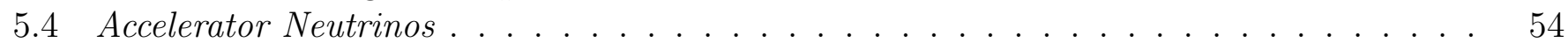

5.5 Reactor Neutrinos . . . . . . . . . . . . . . . . . . . . 56

5.6 Neutrinoless Double $\beta$ Decan . . . . . . . . . . . . . . . . . . . . . . . 57

5.7 Anomalous Magnetic Moment of the Muon . . . . . . . . . . . . . . . 60

$6 \quad$ Weak Probes of the Strong Interaction $\quad 62$

6.1 Hadronic Parity Violation . . . . . . . . . . . . . . . . . . . . . . . . 62

6.2 Probes of Nucleon Strangeness . . . . . . . . . . . . . . . . . . . . . . 66

\begin{tabular}{lll}
\hline 7 & Summary and Outlook & 68
\end{tabular} 


\section{Introduction}

\subsection{Overview}

The study of low energy weak interactions of light quarks and leptons has played an important role in elucidating the structure of the electroweak (EW) interaction. From the early observations of nuclear $\beta$ decay, through the discovery of the neutrino, and up to the observation of neutral current (NC) interactions in atomic parity violation (APV) and parity violating (PV) electron scattering, these studies have contributed decisively to the shape of the Standard Model (SM) [12] as we know it. As of today, the SM has been tested and confirmed at the $0.1 \%$ level in processes for which perturbation theory is applicable, and the absence of any substantial systematic disagreements with the SM - other than the non-vanishing neutrino mass - indicates that whatever physics lies beyond it must be of the decoupling type. Indeed, the search for such "new physics" is now the driving force in particle physics as well as in various subfields of both nuclear and atomic physics.

This search is motivated by both theoretical and experimental considerations. Theoretically, the SM presents a number of unsatisfying features, despite its simplicity and phenomenological successes: the hierarchy problem (instability of the EW scale), the unexplained origins of mass and violation of discrete symmetries (e.g., parity), and the lack of unification with gravity to name a few examples. Similarly, experimental observations of neutrino oscillations, along with cosmological phenomena of dark matter and energy and the matter-antimatter asymmetry, have posed puzzles for particle physics that cannot be solved within the SM. The quest for answers to these questions is clearly one that must be pursued through experiments at high energy colliders, where direct signatures of new particles would be found. At the same time, however, highly precise measurements carried out at lower energies will continue to provide important clues as to the shape of the larger framework in which the SM must be embedded.

Historically, precision EW measurements - at both high and low energies - have provided important insights into various aspects of the SM. For example, the stringent limits obtained on the permanent electric dipole moments of the neutron and neutral atoms imply that the so-called $\theta$-term [4] in the $S U(3)_{C}$ sector of the SM has an unnaturally small coefficient, leading one to suspect the existence of some new symmetry to explain it. From a somewhat different perspective, the study of EW radiative corrections to precision observables and their dependence on the top quark mass, $m_{t}$, led to a predicted range before the top quark was discovered at the Tevatron. The agreement between the measured value of $m_{t}$ and the implications of precision measurements provided a stunning confirmation of the SM at the level of radiative corrections. Finally, the study of weak decays of baryons and mesons taught us that flavor mixing among quarks is rather minimal, in striking contrast to what has emerged for neutrinos from recent neutrino oscillation studies.

In the future, one would like to obtain analogous insights about the structure of what will become the new Standard Model, and in this respect, precision EW measurements performed at a variety of energy scales will continue to be needed. In what follows, we review the status of studies invoving low energy weak interactions of the lightest quarks and leptons and the role they are likely to play in the next decade. After summarizing the status of the SM and briefly reviewing the most widely considered scenarios for physics beyond it, we address various classes of low energy studies that may shed new light on what this physics could be. Our breakdown of these studies includes: NC phenomena, such as neutrino and charged lepton scattering; weak decays; phenomena forbidden or suppressed by symmetries such as CP and lepton flavor; and the properties of neutrinos. We also include the anomalous magnetic moment of the muon, given its recent high visibility as well as its potential sensitivity to new physics (NP) beyond the SM. Among these topics, we especially highlight neutrino physics, which has brought about a revolution in our understanding of the EW interaction in the past few years, and the searches for permanent electric dipole moments, where advances in experimental techniques raise the possibility of another revolution in the next decade. Given the limitations of space for this review, there are also 
topics we have chosen to omit, such as the weak decays and flavor oscillations of heavy quarks. This choice implies no bias on our part regarding the importance of the latter topics, but rather reflects the particular emphasis of this article on the lightest quarks and leptons (for recent reviews of heavy flavor physics, see Refs. [5-10]). We have endeavored to include the most recent experimental results, although it is possible, in the course of compiling this review, we have overlooked developments that occurred after a particular section was written.

Finally, we emphasize that within the SM itself, there remain important elements of both the weak and strong interaction that remain to be tested or more deeply understood. From the standpoint of the EW interaction, one of the most fundamental quantities that has yet to be fully explored is the weak mixing angle, $\theta_{W}$ (defined in subsection 1.2). The scale dependence of gauge couplings above the weak scale can be used to predict the value of $\sin ^{2} \theta_{W}$ at the weak scale in various grand unified theories (GUTs), a feature that has been used - in conjunction with measurements of the weak mixing angle at the $Z$ pole — to test or rule out various scenarios. On the other hand, the SM makes a definite prediction for the running of $\sin ^{2} \theta_{W}$ below the $Z$ pole [1112. Unlike the gauge couplings of QED and QCD, whose running below the weak scale has been stringently tested in a variety of ways, the low scale running of $\sin ^{2} \theta_{W}$ has been tested precisely in only a handful of experiments. This situation is summarized in Figure 1]. To date, only four types of experiments that probe $\sin ^{2} \theta_{W}$ below the $Z$ pole with high precision have been completed or approved: cesium APV [1314, deep-inelastic neutrinonucleus scattering [15], PV Møller scattering [16, and PV elastic electron-proton scattering [17]. Unlike the situation with the QED and QCD couplings, the experimental picture of the low scale running of the weak mixing angle is not entirely settled. There exists a need for additional precision EW measurements at low energies that would further test this fundamental prediction of the SM.

Perhaps a broader arena of SM physics that still presents a variety of challenges to particle and nuclear physicists is that of non-perturbative strong interactions. While the predictions of QCD for perturbative phenomena, heavy quark systems, and chiral dynamics have been tested and confirmed at an impressive level, deep questions remain as to the origins of confinement, the nature of chiral symmetry breaking, and the attendant implications for the quark and gluon substructure of matter. The recent flurry of activity involving baryons with exotic quantum numbers, such as the $\Theta^{+}$(see, e.g., Ref. [18 and references therein), only deepens the sense of mystery that surrounds non-perturbative QCD. While a review of these issues clearly lies outside the purview of this article, we do note that - in the past decade - considerable attention has been paid to the use of the low energy EW interaction as a tool to study certain aspects of non-perturbative QCD. Consequently, we also include a short synopsis of EW probes of the strong interaction at the end of the article.

In the next Subsection, we briefly review the basic structure of the SM, with the main purpose to fix our notation and conventions.

\subsection{The Standard Model}

The Standard Model of the strong and EW interactions is based on gauge interactions with gauge group,

$$
S U(3)_{C} \times S U(2)_{L} \times U(1)_{Y}
$$

where the factors refer to color, isospin, and hypercharge, respectively. The corresponding gauge couplings are denoted by $g_{s} \equiv \sqrt{4 \pi \alpha_{s}}, g$, and $g^{\prime}$. The latter are often traded for the weak mixing angle, $\theta_{W}$, and the electric unit charge, $e$, which are given by,

$$
\begin{aligned}
& \sin ^{2} \theta_{W}=1-\cos ^{2} \theta_{W}=\frac{g^{\prime 2}}{g^{2}+g^{\prime 2}} \\
& e \equiv \sqrt{4 \pi \alpha}=g \sin \theta_{W}=g^{\prime} \cos \theta_{W} .
\end{aligned}
$$

Three generations of fermions are known to transform under the SM gauge group (11) in the representations and with hypercharges as shown in Table 1 for the first generation. Table 1 includes a 


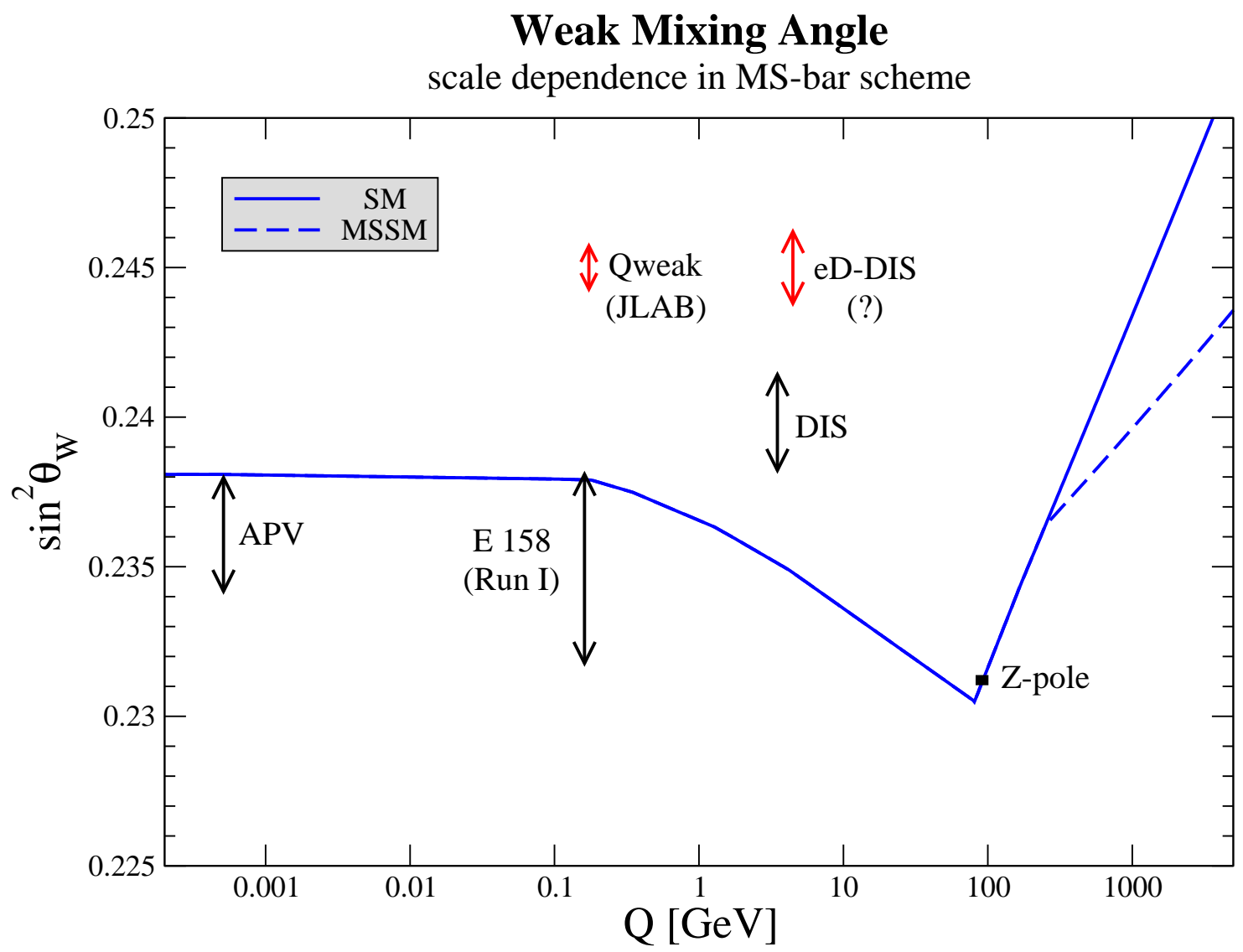

Figure 1: Calculated running of the weak mixing angle in the SM, defined in the $\overline{\mathrm{MS}}$ renormalization scheme (the dashed line indicates the reduced slope typical for the Minimal Supersymmetric Standard Model). Shown are the results from the $Z$ pole, deep inelastic $\nu$ scattering, PV Møller scattering, and APV (Cs and Tl). Qweak and $e$-D DIS refer to a future PV elastic $e^{-}-p$ and a possible deep inelastic $e^{-}$-D measurement, respectively, and have arbitrarily chosen vertical locations.

left-handed anti-neutrino, $\bar{\nu}$, (and correspondingly a right-handed neutrino) which transforms trivially under the SM gauge group, but is needed if one wishes to construct a Dirac neutrino mass term. Since $\bar{\nu}$ does not participate in the SM interactions it is often referred to as sterile. Notice that,

$$
\sum_{\text {quarks }} Y=\sum_{\text {leptons }} Y=0
$$

independently for quarks (lower part of Table 1) and leptons (upper part) of each generation which ensures cancellation of mixed $U(1)_{Y}-S U(3)_{C}$ and $U(1)_{Y}$-gravitational anomalies, respectively. Similarly, the pure $U(1)_{Y}$ and mixed $U(1)_{Y}-S U(2)_{L}$ gauge anomalies cancel by virtue of,

$$
\sum_{\text {all }} Y^{3}=\sum_{\text {doublets }} Y T_{3}^{2}=0
$$

where $T_{3}= \pm 1 / 2$ is the third component of isospin. Gauge invariance forces the gauge vector bosons to transform in the adjoint representation, i.e., the gluons, $G_{\mu}^{\alpha}$, as $(\mathbf{8}, \mathbf{1}, 0)$, the isospin fields, $W_{\mu}^{i}$, as $(\mathbf{1}, \mathbf{3}, 0)$, and the Abelian hypercharge field, $B_{\mu}$, as $(\mathbf{1}, \mathbf{1}, 0)$. 
Table 1: Left-handed fermion representations of the first SM generation. The corresponding right-handed fermions transform in the complex conjugate representations.

\begin{tabular}{|cccc|cccc|}
\hline$\frac{1}{2}\left(1-\gamma_{5}\right) \Psi$ & $S U(3)_{C}$ & $S U(2)_{L}$ & $U(1)_{Y}$ & $\frac{1}{2}\left(1-\gamma_{5}\right) \Psi$ & $S U(3)_{C}$ & $S U(2)_{L}$ & $U(1)_{Y}$ \\
\hline$E=\left(\begin{array}{c}\nu_{e} \\
e^{-}\end{array}\right.$ & $\mathbf{1}$ & $\mathbf{2}$ & $-1 / 2$ & $\bar{\nu}_{e}$ & $\mathbf{1}$ & $\mathbf{1}$ & 0 \\
$Q=\left(\begin{array}{c}u \\
d\end{array}\right)$ & $\mathbf{3}$ & $\mathbf{2}$ & $+1 / 6$ & $\bar{u}$ & $\overline{\mathbf{3}}$ & $\mathbf{1}$ & $-2 / 3$ \\
& & & & $\bar{d}$ & $\overline{\mathbf{3}}$ & $\mathbf{1}$ & $+1 / 3$ \\
\hline
\end{tabular}

In addition, the SM contains a scalar Higgs field, $\phi$, transforming in the $(\mathbf{1}, \mathbf{2},+1 / 2)$ representation, and with potential,

$$
V(\phi)=m_{\phi}^{2} \phi^{\dagger} \phi+\frac{\lambda^{2}}{2}\left(\phi^{\dagger} \phi\right)^{2}
$$

Assuming $m_{\phi}^{2}<0, \phi$ develops a vacuum expectation value (VEV) related to the Fermi constant, $G_{F}$,

$$
|<0| \phi|0>|=\frac{\sqrt{-m_{\phi}^{2}}}{\lambda} \equiv \frac{v}{\sqrt{2}}=\sqrt{\frac{1}{2 \sqrt{2} G_{F}}}=174.104 \pm 0.001 \mathrm{GeV}
$$

and $V(\phi)$ spontaneously breaks part of the gauge symmetry,

$$
S U(2)_{L} \times U(1)_{Y} \rightarrow U(1)_{\mathrm{EM}}
$$

The one remaining physical Higgs degree of freedom, $H=\left(0, \phi^{0} / \sqrt{2}\right)$, acquires a mass given by $M_{H}=$ $\lambda v$. The others become the longitudinal components of the EW gauge bosons,

$$
W_{\mu}^{ \pm}=\frac{1}{\sqrt{2}}\left(W_{\mu}^{1} \mp i W_{\mu}^{2}\right), \quad Z_{\mu}^{0}=\cos \theta_{W} W_{\mu}^{3}-\sin \theta_{W} B_{\mu}
$$

which are the mediators of the weak charged and neutral currents with masses, $M_{W}=T_{3}(\phi) g v=$ $g v / 2=M_{Z} \cos \theta_{W}$. The photon,

$$
A_{\mu}=\cos \theta_{W} B_{\mu}+\sin \theta_{W} W_{\mu}^{3}
$$

remains massless and couples to the electromagnetic charge as given by the Gell-Mann-Nishijima relation, $Q=T_{3}+Y$.

Quarks and charged leptons receive masses through Yukawa interactions, exemplified here for the first generation,

$$
\mathcal{L}_{Y}=\lambda_{e} \bar{E}_{L} \phi e_{R}^{-}+\lambda_{d} \bar{Q}_{L} \phi d_{R}+\lambda_{u} \bar{Q}_{L}\left(i \tau_{2} \phi^{*}\right) u_{R}+\text { h.c. },
$$

where $\tau_{2}$ is a Pauli matrix. For example, the electron mass is given by,

$$
m_{e}=\lambda_{e} \frac{v}{\sqrt{2}}
$$

After symmetry breaking, factors of $1 / \sqrt{2}$ also appear in the effective $H f \bar{f}$-Yukawa couplings, and could be entirely removed by a redefinition of the $\lambda_{f}$. Likewise, one could remove the extra factor of three multiplying the $-i \lambda^{2} H^{4}$ self-coupling. However, with the conventions in Eqs. (5) and (10), $\lambda$ can be directly compared to the gauge couplings (for example, in the context of supersymmetry discussed in Section 1.4) and to $\lambda_{f}$ (for example, in the context of superstring theories and other types of NP 
addressing gauge-Yukawa coupling unification). With these definitions and conventions the fermion Lagrangian takes the form,

$$
\begin{aligned}
\mathcal{L}_{F}=\sum \bar{\psi}_{f} & \left(i \not \partial-m_{f}-\frac{\lambda_{f}}{\sqrt{2}}\right) \psi_{f}-\frac{g}{2 \sqrt{2}} \sum \bar{\psi}_{f} \gamma^{\mu}\left(1-\gamma_{5}\right)\left(T^{+} W_{\mu}^{+}+T^{-} W_{\mu}^{-}\right) \psi_{f} \\
& -e \sum Q_{f} \bar{\psi}_{f} \gamma^{\mu} \psi_{f} A_{\mu}-\frac{\sqrt{g^{2}+g^{\prime 2}}}{2} \sum \bar{\psi} \gamma^{\mu}\left(v_{f}-a_{f} \gamma_{5}\right) \psi Z_{\mu}^{0},
\end{aligned}
$$

where $T^{ \pm}$are the raising and lowering operators of isospin, and where the vector and axial vector $Z$ couplings are given by,

$$
v_{f} \equiv T_{3}^{f}-2 Q_{f} \sin ^{2} \theta_{W}, \quad a_{f} \equiv T_{3}^{f} .
$$

The fact that $\sin ^{2} \theta_{W} \approx 1 / 4$ has two favorable experimental consequences for observables that are proportional to $v_{\ell}$, because for charged leptons $(\ell=e, \mu, \tau) v_{\ell} \ll 1$. They possess an enhanced sensitivity to $\sin ^{2} \theta_{W}$ and, in particular at low energies, possible contributions from NP are relatively enhanced.

In the three-generation SM, the couplings $\lambda_{f}$ become matrix valued. By means of bi-unitary transformations, $U_{L}^{f} \lambda_{f}\left(U_{R}^{f}\right)^{\dagger}$, one passes to the mass eigenstates, producing flavor changing charged currents in the left-handed quark sector through the unitary Cabibbo-Kobayashi-Maskawa matrix [1920,

$$
V_{\mathrm{CKM}}=U_{L}^{u}\left(U_{L}^{d}\right)^{\dagger}
$$

The magnitudes of four of the elements of $V_{\mathrm{CKM}}$ are sufficient to determine the whole matrix up to a single sign ambiguity ${ }^{1}$. Thus, over-constraining $V_{\mathrm{CKM}}$ can provide a valuable test of the SM. This will be addressed in Section 3.3 .

\subsection{Status of the Standard Model}

With the notable exception of the Higgs sector, the basic structure of the SM has been well established since the top quark [21], $t$, and the $\tau$-neutrino [22], $\nu_{\tau}$, were observed directly in the 1990s. Moreover, the $e^{+} e^{-}$colliders LEP 1 and SLC provided very high precision experiments at the $Z$ pole testing many of the relations presented above at the level of quantum corrections. LEP 2 (up to center of mass energies of $209 \mathrm{GeV}$ ) and the Tevatron at Run I (in $p \bar{p}$ collisions at $1.8 \mathrm{TeV}$ ) explored the highest energies to date. It should be stressed, however, that there are many aspects of the SM that are difficult to study at the high energy frontier, but which can be suitably addressed at much lower energies. This is done by exploiting aspects of the weak interaction, such as parity violation, that cleanly separate it from the strong and electromagnetic ones. The purpose of this article is to review these low energy tests; here we give a short account of the experimental situation at high energies.

The first part of Table 2 shows the $Z$ lineshape and leptonic forward-backward (FB) cross section asymmetry measurements from LEP 1 . Besides $M_{Z}$, they include the total $Z$ decay width, $\Gamma_{Z}$, the hadronic cross section on top of the $Z$ resonance, $\sigma_{\text {had }}$, and for each lepton flavor the ratio of hadronic to leptonic partial $Z$ widths, $R_{\ell}$, and the FB-asymmetry,

$$
A_{F B}(\ell)=\frac{3}{4} A_{e} A_{\ell}
$$

defined in terms of the asymmetry parameters,

$$
A_{f}=\frac{2 v_{f} a_{f}}{v_{f}^{2}+a_{f}^{2}}=\frac{1-8 T_{3}^{f} Q_{f} \sin ^{2} \theta_{f}^{\mathrm{eff}}}{1-8 T_{3}^{f} Q_{f} \sin ^{2} \theta_{f}^{\mathrm{eff}}+8 Q_{f}^{2} \sin ^{4} \theta_{f}^{\mathrm{eff}}} .
$$

\footnotetext{
${ }^{1}$ This ambiguity can be resolved by studying $\mathrm{CP}$ violation in the kaon system.
} 
Table 2: Results from precision measurements at the $Z$ pole, and the $W$ boson and top quark masses. Shown are the measurement values compared to the SM prediction obtained from a global analysis of high and low energy experiments using the FORTRAN package GAPP [23. The uncertainties in the SM predictions reflect the uncertainties in the SM parameters, which are determined self-consistently, i.e., we do not use external constraints. This yields, e.g., $\alpha_{s}\left(M_{Z}\right)=0.121 \pm 0.002$. The deviations from the predictions (in terms of the pull) are also shown. The experimental uncertainties of many of the observables are mutually correlated. The largest (anti)-correlations occur between $R_{e}$ and $A_{F B}(e)(-37 \%)$ and between $\sigma_{\text {had }}$ and $\Gamma_{Z}(-30 \%)$ [24]. All experimental correlations are taken into account in the fits. The theoretical uncertainty from unknown higher orders in the prediction for the QCD correction enters commonly $\Gamma_{Z}, \sigma_{\text {had }}$, and the leptonic ratios $R_{\ell}$. We estimate this uncertainty to affect $\alpha_{s}\left(M_{Z}\right)$ at the level of $5 \times 10^{-4}$ [23], which we currently neglect against the much larger experimental errors.

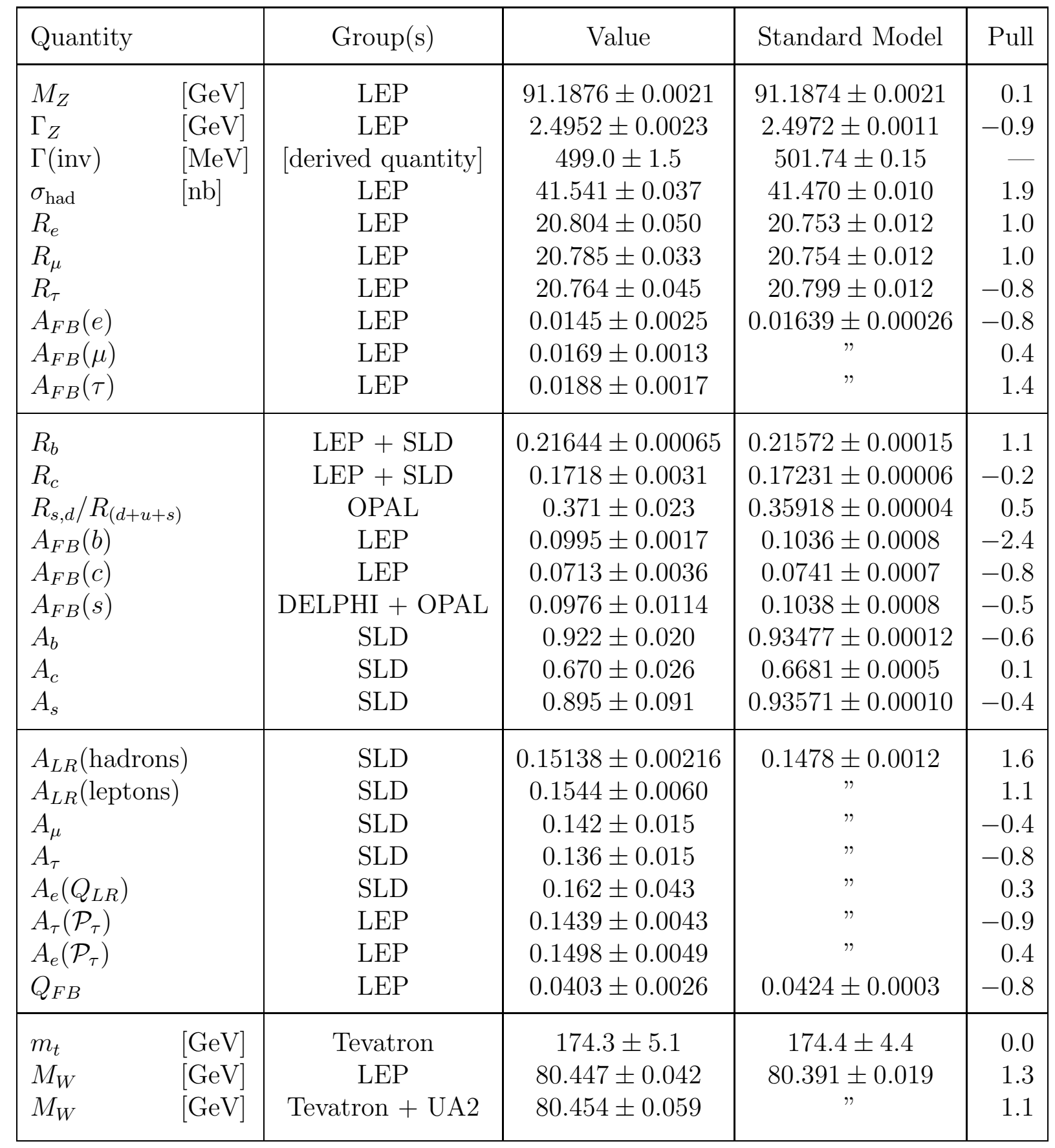


Here, $\sin ^{2} \theta_{f}^{\text {eff }}=\kappa_{f} \sin ^{2} \theta_{W}$ is an effective mixing angle where (flavor dependent) radiative corrections are absorbed into form factors, $\kappa_{f}$. The $\kappa_{f}$ are renormalization-scheme dependent, while the $\sin ^{2} \theta_{f}^{\text {eff }}$ are not. The results in the first part of the Table are obtained simultaneously from a fit to cross section data, and therefore mutually correlated. The invisible $Z$ partial width, $\Gamma$ (inv), is derived from $\Gamma_{Z}, \sigma_{\text {had }}$, and the $R_{\ell}$, and not an independent input. It is smaller by about two standard deviations than the prediction for the SM with three active (participating in the weak interaction) neutrinos. This deviation can be traced to $\sigma_{\text {had }}$ which deviates by a similar amount. Conversely, one can allow the number of active neutrinos, $N_{\nu}$, as an additional free parameter in the fit. This yields $N_{\nu}=2.986 \pm 0.007$ again showing a $2 \sigma$ deviation. The experimental results discussed in this paragraph are final [24].

The second part of Table 2 shows the results from $Z$ decays into heavy flavors 24 ( $b$ and $c$ quarks), as well as into hadrons with non-vanishing strangeness [25 27, but the latter with much poorer precision. For each of these three quark flavors $(q=b, c, s)$ the partial $Z$ width normalized to the hadronic partial width, $R_{q}$, has been obtained, as well as the FB-asymmetry, $A_{F B}(q)$, in analogy to Eq. (15), and the combined left-right (LR) forward-backward asymmetry,

$$
A_{L R}^{F B}(q)=\frac{3}{4} A_{q} .
$$

The heavy flavor results are obtained from a multi-parameter fit including a variety of phenomenological parameters and experimental information from both LEP and the SLC 24]. The results for $q=b$ and $c$ are therefore mutually correlated. $A_{F B}(b)$ is proportional to both $A_{e}$ and $A_{b}$, but since $v_{e} \ll 1$ (see Section 1.2) it is primarily sensitive to $A_{e}$. Similar statements are true of other FB-asymmetries, as well, but $A_{F B}(b)$ stands out because $b$ quark tagging while challenging is easier than tagging of other quarks, and quarks have both bigger cross sections and bigger asymmetries implying smaller statistical uncertainties as compared to leptons. Therefore, $A_{F B}(b)$ provides one of the best determinations of the weak mixing angle. It shows a $2.4 \sigma$ deviation, and (through one-loop radiative corrections) by itself favors larger values for $M_{H}$. It is tempting to suggest effects of NP in the factor $A_{b}$ appearing in $A_{F B}(b)$ to reconcile this deviation and the disagreement with $A_{L R}$ discussed in the following paragraph. However, one would need a $(19 \pm 7) \%$ radiative correction to $\kappa_{b}$ while typical EW radiative corrections are at the sub percent level. NP entering at tree level is generally not resonating ${ }^{2}$ and therefore suppressed relative to the $Z$ contribution. In any case, $R_{b}$ is in reasonable agreement with the SM and one needs to find NP modifying $A_{b}$, but not $R_{b}$, which in general requires some tuning of parameters. Most of the experimental results discussed in this paragraph are still preliminary but close to final [24].

The third part of Table 2 shows further measurements proportional to $v_{\ell}$. The LR cross section asymmetry, $A_{L R}=A_{e}$, from the SLD Collaboration for hadronic [28] and leptonic final states [29] show a combined deviation of $1.9 \sigma$ from the SM prediction. In contrast to $A_{F B}(b)$, it favors small values for $M_{H}$, which are excluded by the direct searches at LEP 2 [30],

$$
M_{H} \geq 114.4 \mathrm{GeV}(95 \% \mathrm{CL}) .
$$

Through LR-FB asymmetries for $\mu$ and $\tau$ final states SLD determines $A_{\mu}$ and $A_{\tau}$ [29] (cf. Eq. (17)) while $A_{e}$ from polarized Bhabba scattering is included in $A_{L R}$ (leptons). The $\tau$ polarization asymmetry, $\mathcal{P}_{\tau}$, is a measurement of $A_{\tau}$, and its angular dependence, $\mathcal{P}_{\tau}^{\mathrm{FB}}$, yields another determination of $A_{e}$ [24]. Finally, the hadronic charge asymmetry at LEP [24] is the weighted sum $^{3}$,

$$
Q_{F B}=\left(\sum_{q=d, s, b}-\sum_{q=u, c}\right) R_{q} A_{F B}(q),
$$

while the LR charge asymmetry from SLD [31] offers a further (less precise) value of $A_{e}$. The experimental results discussed in this paragraph are final 24 .

\footnotetext{
${ }^{2} \mathrm{~A}$ counter example is an extra neutral gauge boson mixing with the ordinary $Z$.

${ }^{3}$ The LEP groups quote results on $Q_{F B}$ as measurements of $\sin ^{2} \theta_{e}^{\text {eff }}$ setting the $R_{q}$ and $A_{q}$ to their SM values.
} 


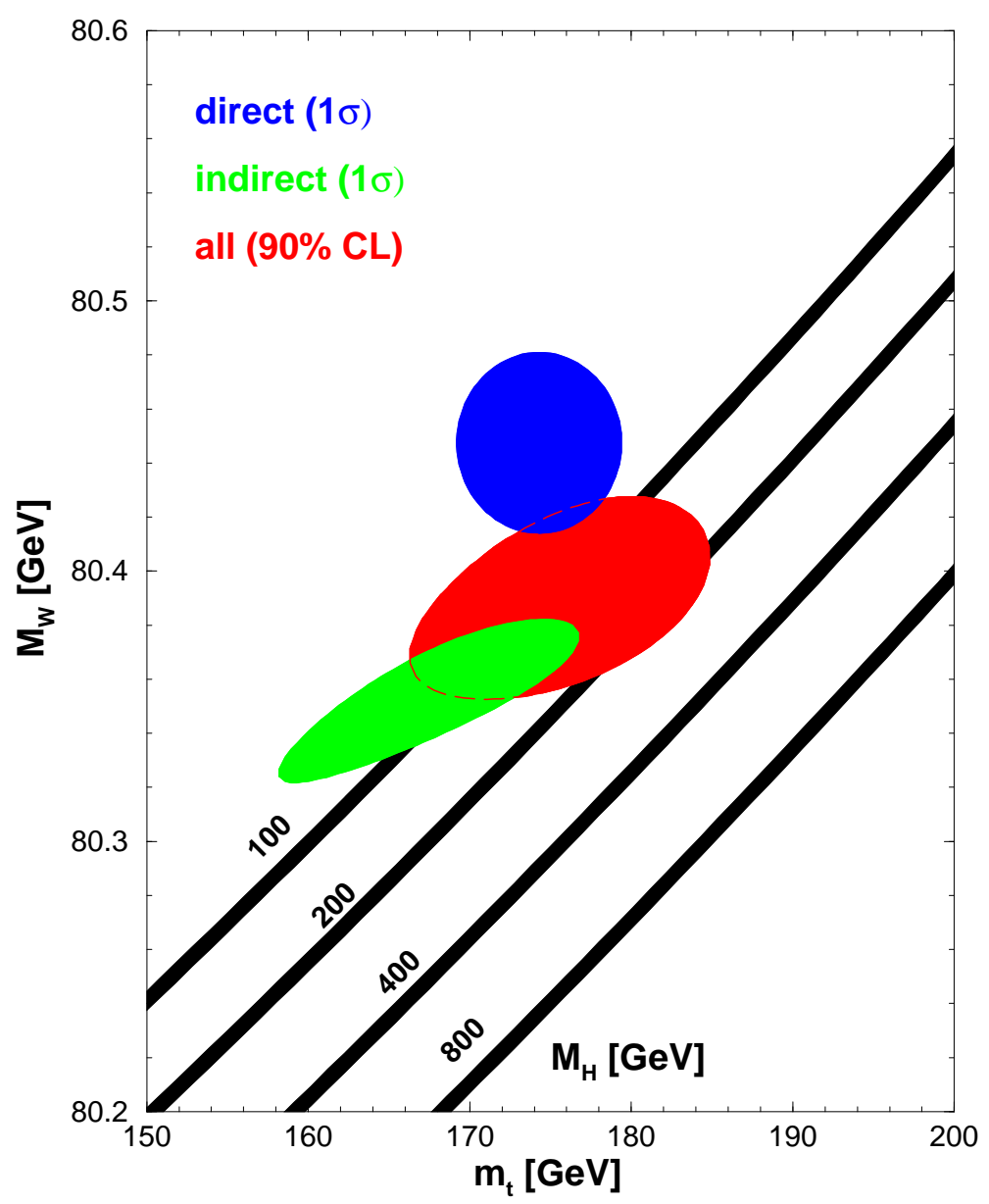

Figure 2: One-standard-deviation $(39.35 \% \mathrm{CL})$ regions in the $M_{W}-m_{t}$ plane for the direct and indirect data. The combined $90 \%$ CL contour $\left(\Delta \chi^{2}=4.605\right)$ is also shown. The widths of the $M_{H}$ bands represent the theoretical uncertainty in the SM prediction $\left(\alpha_{s}\left(M_{Z}\right)=0.120\right)$.

The last part of Table 2 shows the direct $m_{t}$ measurement from the Tevatron 32 33, as well as $M_{W}$ from LEP 2 [24] and $p \bar{p}$ collisions [34-36]. The combined $M_{W}$ is $1.7 \sigma$ higher than the SM expectation. Just as $A_{L R}$ it favors smaller values of $M_{H}$. We compare these mass measurements with all other (indirect) data, and the SM prediction for various values of $M_{H}$ in Figure 2. The definition of $m_{t}$, shown in Table 2 and Figure 2 is the pole mass, which is (approximately) the kinematic mass studied at the Tevatron [37]. For all other quark masses, as well as $m_{t}$ appearing in radiative corrections, the

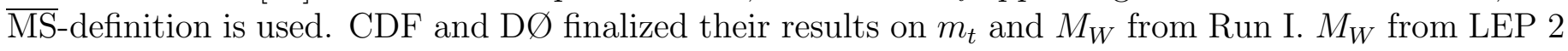
is preliminary and final state interaction effects are still under study. Not shown in Table 2 are the bottom and charm quark masses, $m_{b}$ and $m_{c}$, which enter the SM predictions of numerous observables. In particular, $m_{c}$ is needed to compute $\alpha\left(M_{Z}\right)$ when the renormalization group (RG) based calculation of Ref. [38] is used. Rather than including external constraints, $m_{b}$ and $m_{c}$ are constrained using a set of inclusive QCD sum rules [39] and are recalculated in each call within the fits as functions of $\alpha_{s}$.

The deviations described above may be due to unaccounted for experimental or theoretical effects, physics beyond the SM, or simply fluctuations. New experimental information is needed to clear the situation. Despite of these open problems it must be stressed that the overall agreement between the data and the SM is reasonable. The $\chi^{2}$ per effective degree of freedom of the global fit is $49.1 / 40$ where the probability for a larger $\chi^{2}$ is $15 \%$. One can thus conclude that the SM has been successfully 


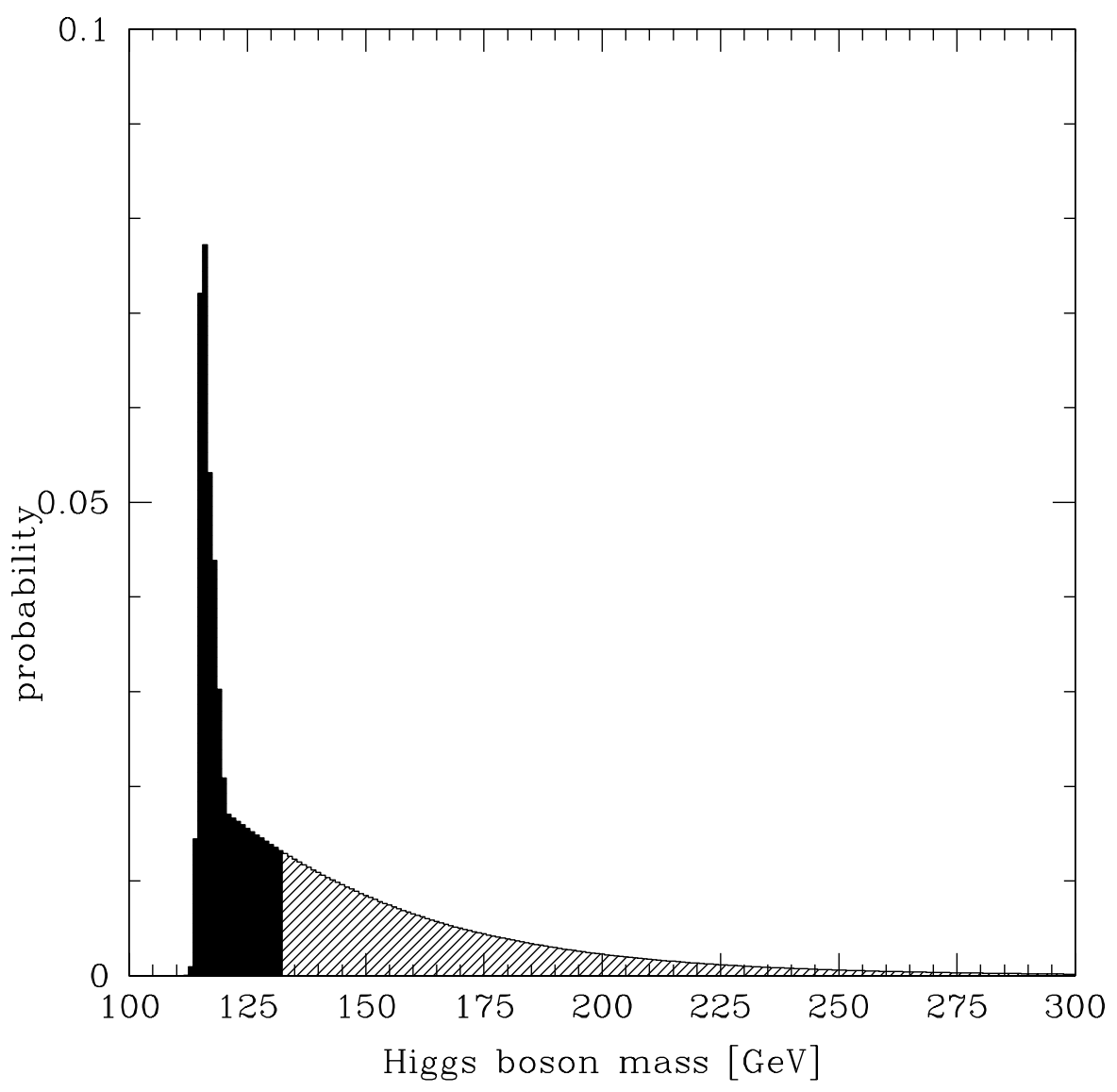

Figure 3: Probability density for $M_{H}$ obtained by combining precision data with direct search results at LEP 2. The peak is due to the candidate Higgs events observed at LEP (updated from Ref. [40]). The full and shaded areas each contain 50\% probability.

tested as the correct theory up to energy scales of $\mathcal{O}\left(M_{Z}\right)$ both at the tree level and at the level of loop corrections. At low energies, NP can therefore only enter as a small perturbation of the SM contribution.

The global fit to all precision data currently favors values for the Higgs boson mass,

$$
M_{H}=85_{-32}^{+49} \mathrm{GeV},
$$

where the central value is slightly below the lower LEP 2 limit (18). If one includes the Higgs search information from LEP 2, one obtains the probability density shown in Figure 3

Note added: After the completion of this Section, a reanalysis of $m_{t}$ from the lepton + jets channel by the D $\varnothing$ Collaboration [41] and the first results from the Tevatron Run II became available. The new average, $m_{t}=177.9 \pm 4.4 \mathrm{GeV}\left[42\right.$, and some new results on $M_{W}$ are driving the extracted $M_{H}$ to values very close to the direct limit (18).

\subsection{Physics Beyond the Standard Model}

Despite the phenomenological success of the SM, it is almost certainly only the low energy approximation of a more fundamental theory. One reason is its arbitrariness, since the gauge group (11) and the fermion (and Higgs) representations in Table 1 are ad hoc, as are the values of the gauge and Yukawa couplings, along with the parameters entering $V_{\mathrm{CKM}}$ and $V(\phi)$. A second reason is that gravity is not included 
in quantized form and it is unlikely that classical General Relativity can coexist (at a fundamental level) with quantum theories of the other interactions. The third class of arguments can be phrased as fine-tuning or naturalness problems, of which there are several within the SM.

By far the most serious one is associated with the cosmological constant, $\Lambda_{\mathrm{C}}$, which on general grounds should be of order the reduced Planck scale, $\kappa_{P}=2.4 \times 10^{18} \mathrm{GeV}$, (the only fundamental mass scale) raised to the forth power. In addition, one expects contributions associated with the EW and QCD phase transition scales, while on the other hand, cosmological observations [44 suggest, $\Lambda_{\mathrm{C}}=(2.3 \pm 0.2 \mathrm{meV})^{4}$. No convincing mechanism is known to explain this small observed value.

Another naturalness problem is related to the topological CP-odd $\theta$-term of QCD [4], which is power counting renormalizable and indeed logarithmically divergent. In the SM this divergence is induced by the complex phase appearing in $V_{\mathrm{CKM}}$. Residually, $\theta \lesssim 10^{-10}$, to account for the limit on the electric dipole moment of ${ }^{199} \mathrm{Hg}$ (see Section 4.1). There is an analogous term for $S U(2)_{L}$, and also for the gravitational interaction [45].

Third, radiative corrections to the $m_{\phi}^{2}$ term in Eq. (5) are quadratically divergent, which strongly suggests one of three logical possibilities: (i) $m_{\phi}^{2}$ is of order $\kappa_{P}$ which is excluded both on formal and phenomenological grounds; (ii) various independent Planck scale contributions to $m_{\phi}^{2}$ conspire to give a residual result not much larger than the weak scale, implying fine tuning at the level of 1 part in $10^{16}$; (iii) physics beyond the SM exists with manifestations at scales not much larger than the weak scale, so as to serve as a regulator for the quadratically divergent loop integrals. Examples for the latter include dynamical symmetry breaking with a composite rather than a fundamental Higgs, supersymmetry (SUSY), and large extra dimensions.

For all these reasons there is generally a strong believe that there is NP beyond the SM. Moreover, the stability of the Higgs potential against large radiative corrections as discussed in the previous paragraph suggests that at least some manifestations of the NP should take place at scales not much larger than $v$.

In this review, we will frequently refer to SUSY [46] as an illustrative example for physics beyond the SM. It solves the problem of quadratically divergent loop corrections 4748 through the introduction of superpartners of the SM particles, which precisely cancel the SM contributions. In fact, in the case of exact (unbroken) SUSY the superpotential which gives rise to Yukawa interactions ${ }^{4}$ is unmodified by radiative corrections by virtue of a non-renormalization theorem [4950]. In simple SUSY ${ }^{5}$ the gauge interactions are logarithmically divergent as in the SM. Thus, gauge couplings still evolve according to the RG, but unlike in the SM, in the MSSM they approximately converge to a common value at a scale $M_{U} \approx 2 \times 10^{16} \mathrm{GeV}[52 \sqrt[54]{54}$.

As for the SUSY Higgs sector, the $\lambda_{u}$-term in Eq. (10) can not be supersymmetrized (complex conjugation is not a supersymmetric operation), and therefore a second Higgs doublet, $H_{u}$, with opposite hypercharge to the standard Higgs, $H_{d} \equiv \phi$, needs to be introduced for up-type quark mass terms. This is also necessary to cancel the gauge anomaly contribution of the Higgs-fermions (Higgsinos). As a consequence, there are now five physical Higgs degrees of freedom: the two CP-even, $H_{1,2}$, with $M_{H_{1}} \leq M_{H_{2}}$ by convention, the CP-odd, $A$, and the charged pair, $H^{ \pm}$.

In the minimal supersymmetric SM (MSSM), the $\lambda^{2}$-term in Eq. (5) arises from the supersymmetric gauge kinetic terms, leading to the prediction,

$$
\lambda=T_{3}(\phi) \sqrt{g^{2}+g^{\prime 2}}
$$

The mass term in Eq. (5) is replaced by a bilinear term, $\mu H_{u} H_{d}$, in the superpotential. Again, $\mu \ll \kappa_{P}$ is assumed rather than predicted, but now this hierarchy is technically natural, i.e., stable under radiative

\footnotetext{
${ }^{4}$ The superpotential versions of both $\mathcal{L}_{Y}$ and $\mathcal{L}_{Y}^{\text {SUSY }}$ discussed later also contribute to the scalar potential, but this will not affect the discussion in this review.

${ }^{5}$ Theories of extended four-dimensional SUSY do not allow chiral fermions and are therefore excluded as effective theories in four dimensions [51].
} 
corrections. Since $\lambda$ is no longer a free parameter, the Higgs boson masses are calculable from other inputs, and one obtains the tree level upper bound $M_{H_{1}} \leq M_{Z}$. Radiative corrections [55]59] move this bound to 60],

$$
M_{H_{1}} \lesssim 134 \pm 5 \pm 3_{-0}^{+2} \mathrm{GeV},
$$

where the first error is induced by $m_{t}$, the second is the theory uncertainty in the prediction, and the last one corresponds to shifting the SUSY breaking scale (see next paragraph) from 1 to $2 \mathrm{TeV}$.

The non-observation of the superpartners implies that SUSY is broken. The mechanism of SUSY breaking is one of the outstanding theoretical problems, and is currently treated phenomenologically by allowing the most general set 61] of soft SUSY breaking parameters: bilinear scalar masses, $m_{i j}^{2}$, gauge fermion (gaugino) masses, $M_{i}$, and trilinear scalar couplings, $A_{i j k}$. The quantity $\mu$ and various of the soft SUSY breaking parameters are assumed to trigger EW symmetry breaking, but within the MSSM it is not understood why $\mu$ is of the order the soft masses 62 (the so-called $\mu$-problem). The VEV, $v$, now receives contributions from both Higgs doublets, $v^{2}=v_{1}^{2}+v_{2}^{2}$, with $v_{1} / v_{2} \equiv \tan \beta$.

With the gauge and particle choice of the SM, baryon number, $B$, and lepton number, $L$, are conserved, ensuring a stable proton lifetime. In the MSSM, however, additional Yukawa couplings to the ones in Eq. (10) are allowed by the symmetries of the model,

$$
\mathcal{L}_{Y}^{\mathrm{SUSY}}=\lambda \bar{E}_{L} E_{L} e_{R}^{-}+\lambda^{\prime} \bar{Q}_{L} E_{L} d_{R}+\lambda^{\prime \prime} \bar{u}_{R} \bar{d}_{R} \bar{d}_{R}+\mu^{\prime} \bar{E}_{L} H_{u}
$$

where any one factor in the trilinear couplings is bosonic, while the other two are fermionic. The first and the third term in Eq. (23) are antisymmetric in the $S U(2)$ and $S U(3)$ indices, respectively, so that they need to be antisymmetrized with respect to family space, as well. The third term violates $B$, the others violate $L$. One can eliminate all terms in Eq. (23) by a so-called $R$-symmetry ${ }^{6}$ [6364, which is consistent with SUSY although fermions transform differently under it than their scalar partners. If $R_{P}$ is exact, the lightest supersymmetric particle would be stable and a cold dark matter candidate. If $R_{P}$ is broken, $B$ or $L$ or both would be violated. For more details on SUSY and its phenomenology, see the reviews 6566 and 6768 , respectively.

Another possible type of NP involves additional gauge interactions, in particular extra Abelian group factors. An extra $U(1)^{\prime}$ by itself does not solve any of the problems associated with the SM. But additional $U(1)^{\prime}$ symmetries are predicted in many extensions of the SM, including technicolor 69], Grand Unified Theories [70], SUSY, Kaluza-Klein theories [71, or string theories [7273. Moreover, they can solve the $\mu$-problem of the MSSM [74, and forbid the terms in Eq. (23). They generally do not spoil the successful gauge coupling unification, and $U(1)^{\prime}$ symmetries can be found addressing all these issues simultaneously, while at the same time being free of anomalies [75]. The precision $Z$ pole observables discussed in Section 1.3 mainly constrain the mixing of the corresponding $Z^{\prime}$ with the ordinary $Z$. Low energy observables, especially from APV as discussed in Section 2.3. provide important mass and coupling constraints on potential $Z^{\prime}$ s. We now turn to a detailed discussion of these observables.

\section{Weak Neutral Current Phenomena}

\subsection{Neutrino Scattering}

Assuming an arbitrary gauge theory, one can write the four-Fermi interaction Lagrangian for the weak processes discussed in this and in the next Section in model independent form. For NC neutrino scattering one has,

$$
\mathcal{L}_{\mathrm{NC}}^{\nu f}=-\frac{G_{F}}{\sqrt{2}} \bar{\nu} \gamma^{\mu}\left(1-\gamma_{5}\right) \nu \bar{f} \gamma_{\mu}\left[\epsilon_{L}(f)\left(1-\gamma_{5}\right)+\epsilon_{R}(f)\left(1+\gamma_{5}\right)\right] f,
$$

\footnotetext{
${ }^{6}$ To this end, it is sufficient to employ the discrete $R$-parity subgroup, $R_{P}=(-1)^{3 B+L+2 S}$, where $S$ refers to spin.
} 
where the SM tree level prediction for the effective couplings can be obtained from Eqs. (12) and (13),

$$
\epsilon_{L}(f)=T_{3}^{f}-Q_{f} \sin ^{2} \theta_{W}, \quad \epsilon_{R}(f)=-Q_{f} \sin ^{2} \theta_{W} .
$$

EW radiative corrections [117677] modify Eq. (25) through the NC $\rho$-parameter, which appears when the $Z$ propagator is expressed in terms of $G_{F}$, through the RG evolution of the weak mixing angle, and through further propagator, vertex, and box contributions.

Historically, deep inelastic scattering (DIS) of neutrinos off nuclei, $\nu_{\mu} N \rightarrow \nu_{\mu} X$, with $X$ an arbitrary hadronic final state, provided the discovery of the weak $\mathrm{NC}\left[78\right.$. For measurements of $\sin ^{2} \theta_{W}$, it is advantageous to choose $N$ approximately isoscalar. Many theoretical uncertainties cancel in the ratio of neutral-to-charged current cross sections [79],

$$
R_{\nu}=\frac{\sigma_{\nu N}^{\mathrm{NC}}}{\sigma_{\nu N}^{\mathrm{CC}}}=\frac{\sigma\left(\nu_{\mu} N \rightarrow \nu_{\mu} X\right)}{\sigma\left(\nu_{\mu} N \rightarrow \mu^{-} X\right)}=g_{L}^{2}+r g_{R}^{2}, \quad R_{\bar{\nu}}=\frac{\sigma_{\bar{\nu} N}^{\mathrm{NC}}}{\sigma_{\bar{\nu} N}^{\mathrm{CC}}}=\frac{\sigma\left(\bar{\nu}_{\mu} N \rightarrow \bar{\nu}_{\mu} X\right)}{\sigma\left(\bar{\nu}_{\mu} N \rightarrow \mu^{+} X\right)}=g_{L}^{2}+\frac{g_{R}^{2}}{\bar{r}},
$$

where at tree level and ignoring sea quarks,

$$
g_{L}^{2}=\epsilon_{L}(u)^{2}+\epsilon_{L}(d)^{2}=\frac{1}{2}-\sin ^{2} \theta_{W}+\frac{5}{9} \sin ^{4} \theta_{W}, \quad g_{R}^{2}=\epsilon_{R}(u)^{2}+\epsilon_{R}(d)^{2}=\frac{5}{9} \sin ^{4} \theta_{W} .
$$

$r$ and $\bar{r}$ are related to the charged current (CC) ratio,

$$
r \sim \frac{1}{\bar{r}} \sim \frac{\sigma_{\bar{\nu} N}^{\mathrm{CC}}}{\sigma_{\nu N}^{\mathrm{CC}}} \sim \frac{1}{2}
$$

which depends on the experimental details and is measured directly. $R_{\nu}$ is more sensitive to the weak mixing angle than $R_{\bar{\nu}}$, and both are sensitive to charm threshold effects which introduce the dominant theoretical uncertainty. This uncertainty (and some others) largely cancels in the ratio [80],

$$
R^{-}=\frac{\sigma_{\nu N}^{\mathrm{NC}}-\sigma_{\bar{\nu} N}^{\mathrm{NC}}}{\sigma_{\nu N}^{\mathrm{CC}}-\sigma_{\bar{\nu} N}^{\mathrm{CC}}}=\frac{R_{\nu}-r R_{\bar{\nu}}}{1-r}=g_{L}^{2}-g_{R}^{2}=\frac{1}{2}-\sin ^{2} \theta_{W}
$$

which was used by the NuTeV Collaboration [15 to measure the weak mixing angle precisely off the $Z$ pole. NuTeV was the first experiment of this type with a clean $\bar{\nu}_{\mu}$-beam at its disposal which is necessary to measure $R^{-}$. In the presence of NP, however, which will in general affect $\nu_{\mu}$ and $\bar{\nu}_{\mu}$ cross sections differently, one should rather monitor $R_{\nu}$ and $R_{\bar{\nu}}$ independently, or equivalently, $g_{L}^{2}$ and $g_{R}^{2}$. The uncertainty from the charm threshold is modeled using the slow rescaling prescription [8182] parametrized using an effective charm mass, $m_{c}^{\text {eff }}$. In practice, $\mathrm{NuTeV}$ fits to $g_{L}^{2}, g_{R}^{2}$, and $m_{c}^{\text {eff }}$ with an external constraint. As is shown in Table 3, there is a $2.9 \sigma$ deviation in $g_{L}^{2}$, reflecting the quoted $3 \sigma$ deviation [15] in the weak mixing angle.

There are various attempts to explain the apparent deviation of the $\mathrm{NuTeV}$ result from the SM prediction. It cannot be excluded that nuclear shadowing effects are large enough to induce shifts comparable to the $\mathrm{NuTeV}$ discrepancy 88. However, both $R_{\nu}$ and $R_{\bar{\nu}}$ are seen lower than the SM prediction, while nuclear shadowing — when modeled using vector meson dominance - predicts a larger reduction in the $\mathrm{CC}$ than in the $\mathrm{NC}$ [89]. Moreover, the deviation in $R_{\nu}$ is larger than the one in $R_{\bar{\nu}}$ while from nuclear shadowing one would expect the opposite. Shadowing and other nuclear effects are also discussed in Ref. 90. An asymmetry in the first moments of the strange sea distributions, $S-\bar{S}$, could reduce the discrepancy by as much as 50\% 91], although $\mathrm{NuTeV}$ finds a very small asymmetry from a next-to-leading order (NLO) analysis of dimuon production cross sections 92. Isospin symmetry violation 94, particularly violation of charge symmetry under which protons and neutrons and simultaneously $u$ and $d$ quarks are interchanged, can possibly be responsible for the NuTeV effect. However, firm conclusions cannot be drawn before a global analysis of parton distribution functions (PDFs) 
Table 3: Results from deep inelastic neutrino-nucleus scattering. Shown are the measurement values, the SM prediction and the pull as in Table 2. The second errors (where shown) are theoretical. The experimental results are updates of the original CDHS 83] and CHARM 84 publications for which we used the analysis of Ref. 85]. The coefficients entering the linear combinations (30) of the various measurements are given in Table 4 .

\begin{tabular}{|l|c|c|c|r|}
\hline Quantity & Group & Value & Standard Model & Pull \\
\hline$g_{L}^{2}$ & NuTeV & $0.30005 \pm 0.00137$ & $0.30397 \pm 0.00023$ & -2.9 \\
$g_{R}^{2}$ & NuTeV & $0.03076 \pm 0.00110$ & $0.03005 \pm 0.00004$ & 0.6 \\
$R^{\nu}$ & CCFR & $0.5820 \pm 0.0027 \pm 0.0031$ & $0.5833 \pm 0.0004$ & -0.3 \\
$R^{\nu}$ & CDHS & $0.3096 \pm 0.0033 \pm 0.0028$ & $0.3092 \pm 0.0002$ & 0.1 \\
$R^{\nu}$ & CHARM & $0.3021 \pm 0.0031 \pm 0.0026$ & & -1.7 \\
$R^{\bar{\nu}}$ & CDHS & $0.384 \pm 0.016 \pm 0.007$ & $0.3862 \pm 0.0002$ & -0.1 \\
$R^{\bar{\nu}}$ & CHARM & $0.403 \pm 0.014 \pm 0.007$ & & 1.0 \\
$R^{\bar{\nu}}$ & CDHS (1979) & $0.365 \pm 0.015 \pm 0.007$ & $0.3817 \pm 0.0002$ & -1.0 \\
\hline
\end{tabular}

allowing departures from isospin symmetry and an asymmetric strange sea has been performed [95]. A similar remark applies to QCD corrections which could have significant impact on the individual ratios $R_{\nu}$ and $R_{\bar{\nu}}$ (but not $R^{-}$) 91]: NuTeV currently uses leading order (LO) expressions and LO PDFs; conclusions about the size of QCD corrections must await a NLO analysis including NLO PDFs. For recent discussions of QCD corrections and parton structure relevant to NuTeV, see Refs. 9697.

If none of the SM effects discussed in the previous paragraph are ultimately able to explain the $\mathrm{NuTeV}$ results, they might be due to NP (or a fluctuation). Most NP scenarios have difficulty explaining the NuTeV deviation. For example, inclusion of supersymmetric radiative corrections as well as $R_{P}$ violating (RPV) interactions generally tend to increase the value of $R^{\nu}$ and is unlikely to help account for the NuTeV results 98. A $Z^{\prime}$ boson would affect the $\epsilon_{L, R}(f)$ couplings, but it is difficult to explain the entire discrepancy that way, unless one chooses a family non-universal $Z^{\prime}$ [99] with carefully tuned couplings, which could also shed light on some of the other anomalies 100 discussed in Section 1.3. Similarly, one might invoke triplet leptoquarks with carefully chosen mass splittings [91. A $\nu_{\mu}$-mixing with an extra heavy neutrino could also be the cause, but this would imply shifts in other observables, as well, so that this scenario 101102 requires a conspiracy of effects to obtain an overall consistent picture.

The other results in Table 3 are only very approximately proportional to $R_{\nu}$ or $R_{\bar{\nu}}$. In practice, the measured quantities are the linear combinations of the $\epsilon_{L, R}^{2}(q)$ [83],

$$
R^{\nu(\bar{\nu})}=(1-\delta)\left[a_{L}(u) \epsilon_{L}^{2}(u)+a_{L}(d) \epsilon_{L}^{2}(d)+a_{R}(u) \epsilon_{R}^{2}(u)+a_{R}(d) \epsilon_{R}^{2}(d)\right],
$$

where $\delta$ accounts for the purely photonic (QED) radiative corrections. In addition to the charm threshold and radiative corrections, the interpretation of $\nu$-nucleus DIS experiments is complicated by corrections for and uncertainties from non-isoscalarities, structure functions, sea quark effects, etc., which also gives rise to theoretical correlations among the results. The residual correlation between $g_{L}^{2}$ and $g_{R}^{2}$ from $\mathrm{NuTeV}$ is accidentally very small $(-0.017 \%)$. This and the correlations between the older results are taken into account, while the correlations between $\mathrm{NuTeV}$ and the other experiments are currently neglected. The results of the older experiments should also be updated with the more precise $\mathrm{NuTeV}$ results on $m_{c}^{\text {eff }}$ and the quark sea, and if possible, with more recent structure functions.

Initially, elastic $\nu$ - $p$ scattering [104] also served as a SM test and as a measurement of $\sin ^{2} \theta_{W}$. The most precise result [105], $\sin ^{2} \theta_{W}=0.218_{-0.047}^{+0.039}$, is no longer competitive with present day determinations, 
Table 4: Coefficients defining the linear combinations in Eq. (30). CHARM has been adjusted to be directly comparable to CDHS. The average momentum transfer, $Q^{2}=-q^{2}$, is also shown. In the case of $\mathrm{NuTeV}$ it corresponds to the geometric mean between the average $\log Q^{2}$-values of neutrino and anti-neutrino reactions. In the case of the CCFR [87] and $\mathrm{NuTeV}$ experiments, $\delta$ has been absorbed into the $\epsilon$ parameters.

\begin{tabular}{|l|c|c|c|c|c|c|r|}
\hline Quantity & Group(s) & $\delta$ & $Q^{2}\left[\mathrm{GeV}^{2}\right]$ & $a_{L}(u)$ & $a_{L}(d)$ & $a_{R}(u)$ & $a_{R}(d)$ \\
\hline$g_{L}^{2}$ & NuTeV & 0 & 12 & 1 & 1 & 0 & 0 \\
$g_{R}^{2}$ & NuTeV & 0 & 12 & 0 & 0 & 1 & 1 \\
$R^{\nu}$ & CCFR & 0 & 35 & 1.698 & 1.881 & 1.070 & 1.226 \\
$R^{\nu}$ & CDHS + CHARM & 0.023 & 21 & 0.936 & 1.045 & 0.379 & 0.453 \\
$R^{\bar{\nu}}$ & CDHS + CHARM & 0.026 & 11 & 0.948 & 1.134 & 2.411 & 2.690 \\
$R^{\bar{\nu}}$ & CDHS (1979) & 0.024 & 11 & 0.944 & 1.126 & 2.295 & 2.563 \\
\hline
\end{tabular}

but elastic $\nu$ scattering continues to play a crucial role in form factor measurements (see Section 6.2).

$\mathrm{NC} \nu$-e scattering [106] is described by the Lagrangian (24) with $f=e$, and one usually extracts,

$$
g_{V}^{\nu e}=\epsilon_{L}(e)+\epsilon_{R}(e)=-\frac{1}{2}+2 \sin ^{2} \theta_{W}, \quad g_{A}^{\nu e}=\epsilon_{L}(e)-\epsilon_{R}(e)=-\frac{1}{2} .
$$

EW radiative corrections to $g_{V, A}^{\nu e}$ have been obtained in Refs. 107108. The results are summarized in Table 5. $\nu_{e}$ scattering has been studied at LANL [14 115] and $\bar{\nu}_{e}$ scattering at the Savannah River plant [116. In these cases one has to add the CC Lagrangian so that effectively, $\epsilon_{L}(e) \rightarrow \epsilon_{L}(e)+1$, and, $g_{V, A}^{\nu e} \rightarrow g_{V, A}^{\nu e}+1$. The basic observables are the cross sections, which in the limit of large incident $\nu$ energies, $E_{\nu} \gg m_{e}$, read,

$$
\sigma=\frac{G_{F}^{2} m_{e} E_{\nu}}{2 \pi}\left[\left(g_{V}^{\nu e} \pm g_{A}^{\nu e}\right)^{2}+\frac{1}{3}\left(g_{V}^{\nu e} \mp g_{A}^{\nu e}\right)^{2}\right]
$$

where the upper (lower) sign refers to (anti-)neutrinos. Some experiments achieved slight improvements by also including differential cross section information. The NC-CC interference in $\nu_{e}-e$ scattering resolves a sign ambiguity, $g_{V, A}^{\nu e} \rightarrow-g_{V, A}^{\nu e}$, relative to the CC coupling, and is found in agreement with the SM [114. A new reactor-based elastic $\bar{\nu}_{e}-e^{-}$scattering experiment has been suggested in Ref. [103], aiming at an improvement by a factor of four in $\sin ^{2} \theta_{W}$ relative to the results in Table 5 .

\subsection{Charged Lepton Scattering}

The parity $(\mathrm{P})$ or charge-conjugation ${ }^{7}(\mathrm{C})$ violating NC Lagrangian for charged lepton-hadron scattering is given by (assuming lepton-universality),

$$
\mathcal{L}_{\mathrm{NC}}^{\nu f}=\frac{G_{F}}{\sqrt{2}} \sum_{q}\left[C_{1 q} \bar{\ell} \gamma^{\mu} \gamma_{5} \ell \bar{q} \gamma_{\mu} q+C_{2 q} \bar{\ell} \gamma^{\mu} \ell \bar{q} \gamma_{\mu} \gamma_{5} q+C_{3 q} \bar{\ell} \gamma^{\mu} \gamma_{5} \ell \bar{q} \gamma_{\mu} \gamma_{5} q\right]
$$

where the effective couplings at the SM tree level are again obtained from Eqs. (12) and (13),

$$
C_{1 q}=-T_{3}^{q}+2 Q_{q} \sin ^{2} \theta_{W}, \quad C_{2 u}=-C_{2 d}=-\frac{1}{2}+2 \sin ^{2} \theta_{W}, \quad C_{3 u}=-C_{3 d}=\frac{1}{2} .
$$

${ }^{7}$ We refer here to the conjugation of the lepton charge, and not the fundamental charge-conjugation operation which would also require replacing the target by anti-matter. 
Table 5: $\nu_{\mu}$-e scattering results from CHARM [109] and CHARM II [110] at CERN and from E734 [111] at BNL, obtained from two-parameter fits. The E734 Collaboration reports a $16.3 \%$ statistical anti-correlation and a $41.3 \%$ systematic correlation between $g_{V}^{\nu e}$ and $g_{A}^{\nu e}$, for a total 11.9\% correlation. Only the world averages (which are updated from Ref. [112]) are used as constraints in the fits. These also include results from $\nu_{e}-e$ scattering, as well as older $\nu_{\mu}$-e experiments. The overall correlation of the world averages is $-5 \%$.

\begin{tabular}{|c|c|c|c|c|}
\hline Quantity & Group(s) & Value & Standard Model & Pull \\
\hline$g_{V}^{\nu e}$ & CHARM & $-0.060 \pm 0.073$ & $-0.0398 \pm 0.0003$ & - \\
$g_{V}^{\nu e}$ & E 734 & $-0.107 \pm 0.046$ & & - \\
$g_{V}^{\nu e}$ & CHARM II & $-0.035 \pm 0.017$ & & - \\
$g_{V}^{\nu e}$ & world average & $-0.041 \pm 0.015$ & & -0.1 \\
\hline$g_{A}^{\nu e}$ & CHARM & $-0.570 \pm 0.072$ & $-0.5065 \pm 0.0001$ & - \\
$g_{A}^{\nu e}$ & E 734 & $-0.514 \pm 0.036$ & & - \\
$g_{A}^{\nu e}$ & CHARM II & $-0.503 \pm 0.017$ & & - \\
$g_{A}^{\nu e}$ & world average & $-0.507 \pm 0.014$ & & 0.0 \\
\hline
\end{tabular}

The $C_{3 q}$ are $\mathrm{P}$ conserving, but $\mathrm{C}$ violating couplings affecting asymmetries involving charge reversal. Alternatively, the following linear combinations [117] motivated by isospin symmetry are also used,

$$
\begin{aligned}
& \tilde{\alpha}=C_{1 u}-C_{1 d}=-1+2 \sin ^{2} \theta_{W}, \quad \tilde{\gamma}=C_{1 u}+C_{1 d}=\frac{2}{3} \sin ^{2} \theta_{W}, \\
& \tilde{\beta}=C_{2 u}-C_{2 d}=-1+4 \sin ^{2} \theta_{W}, \quad \tilde{\delta}=C_{2 u}+C_{2 d}=0 \text {. }
\end{aligned}
$$

EW radiative corrections to the couplings in Eq. (34) other than the $C_{3 q}$ have first been obtained in Refs. [118 119] and updated in Ref. [120].

The terms in Eq. (33) give rise to interference effects [121122 with the parity conserving QED terms in Eq. (12), which can be isolated by measuring parity violating LR asymmetries or by comparing processes related by charge-conjugation in charged lepton-hadron scattering. Currently the most precise results are obtained from APV which will be discussed in the next Subsection 2.3.

Historically, the SLAC e-D scattering experiment [123] was crucial in establishing the SM. It measured [124] the cross section asymmetry [125 128,

$$
\frac{\sigma_{R}-\sigma_{L}}{\sigma_{R}+\sigma_{L}}=\frac{3 G_{F} Q^{2}}{10 \sqrt{2} \pi \alpha}\left[\left(2 C_{1 u}-C_{1 d}\right)+g(y)\left(2 C_{2 u}-C_{2 d}\right)\right]
$$

where $y=\left(E_{0}-E^{\prime}\right) / E_{0}$, where $E_{0}\left(E^{\prime}\right)$ is the incident (scattered) electron energy, and where ${ }^{8}$,

$$
g(y)=\frac{1-(1-y)^{2}}{1+(1-y)^{2}}
$$

Eq. (36) is valid for isoscalar targets and neglects $s$ quarks, $c$ quarks, and anti-quarks. The only experiment to date which has measured a charge-conjugation cross section asymmetry [129 130],

$$
\frac{\sigma^{+}(-|\lambda|)-\sigma^{-}(+|\lambda|)}{\sigma^{+}(-|\lambda|)+\sigma^{-}(+|\lambda|)}=-\frac{G_{F} Q^{2}}{10 \sqrt{2} \pi \alpha} g(y)\left[\left(2 C_{3 u}-C_{3 d}\right)+\lambda\left(2 C_{2 u}-C_{2 d}\right)\right],
$$

\footnotetext{
${ }^{8}$ For more precise measurements, $g(y)$ needs to be corrected for the longitudinal contributions to $\gamma$ and $Z$ exchange.
} 
Table 6: Observables sensitive to the $P$ or $C$ violating coefficients $C_{i q}$. The errors are the combined (in quadrature) statistical, systematic and theoretical uncertainties. The first two lines result from a fit to 11 different kinematic points (a 5\% uncertainty in the polarization was common to all points) and have a $-92.7 \%$ correlation. Including a $7 \%$ theory uncertainty [141] increases the error in the first line to \pm 0.18 and decreases the correlation to $-86.6 \%$. The two CERN entries are for muon beam energies (polarizations) of $120 \mathrm{GeV}$ $(66 \%)$ and $200 \mathrm{GeV}(81 \%)$, respectively. Assuming 100\% correlated systematic errors yields a correlation of $17.4 \%$ between them. The second line (SLAC) contains a $31.6 \%$ correction to account for sea quarks, while the corresponding correction is $7.5 \%$ for CERN [141]. The Mainz result includes a 10\% theory error [141].

\begin{tabular}{|c|c|c|c|c|c|}
\hline Beam & Process & $\overline{Q^{2}}\left[\mathrm{GeV}^{2}\right]$ & Combination & Result/Status & SM \\
\hline SLAC & $e^{-}$-D DIS & 1.39 & $2 C_{1 u}-C_{1 d}$ & $-0.90 \pm 0.17$ & -0.7185 \\
SLAC & $e^{-}$-D DIS & 1.39 & $2 C_{2 u}-C_{2 d}$ & $+0.62 \pm 0.81$ & -0.0983 \\
CERN & $\mu^{ \pm}$-C DIS & 34 & $0.66\left(2 C_{2 u}-C_{2 d}\right)+2 C_{3 u}-C_{3 d}$ & $+1.80 \pm 0.83$ & +1.4351 \\
CERN & $\mu^{ \pm}$-C DIS & 66 & $0.81\left(2 C_{2 u}-C_{2 d}+2 C_{3 u}-C_{3 d}\right.$ & $+1.53 \pm 0.45$ & +1.4204 \\
Mainz & $e^{-}$-Be QE & 0.20 & $2.68 C_{1 u}-0.64 C_{1 d}+2.16 C_{2 u}-2.00 C_{2 d}$ & $-0.94 \pm 0.21$ & -0.8544 \\
Bates & $e^{-}$-C elastic & 0.0225 & $C_{1 u}+C_{1 d}$ & $0.138 \pm 0.034$ & +0.1528 \\
Bates & $e^{-}$-D QE & 0.1 & $C_{2 u}-C_{2 d}$ & $0.015 \pm 0.042$ & -0.0624 \\
JLAB & $e^{-}-p$ elastic & 0.03 & $2 C_{1 u}+C_{1 d}$ & approved & +0.0357 \\
SLAC & $e^{-}$-D DIS & 20 & $2 C_{1 u}-C_{1 d}$ & to be proposed & -0.7185 \\
SLAC & $e^{-}$-D DIS & 20 & $2 C_{2 u}-C_{2 d}$ & to be proposed & -0.0983 \\
SLAC & $e^{ \pm}$-D DIS & 20 & $2 C_{3 u}-C_{3 d}$ & to be proposed & +1.5000 \\
\hline- & 133 Cs APV & 0 & $-376 C_{1 u}-422 C_{1 d}$ & $-72.69 \pm 0.48$ & -73.16 \\
- & 205 Tl APV & 0 & $-572 C_{1 u}-658 C_{1 d}$ & $-116.6 \pm 3.7$ & -116.8 \\
\hline
\end{tabular}

was the CERN $\mu^{ \pm} \mathrm{C}$ scattering experiment 131. In this experiment, the $\mu$-polarization, $\lambda$, was reserved simultaneously with the $\mu$-charge. A linear combination of the $C_{1 q}$ and $C_{2 q}$ different from those entering DIS was obtained in an experiment at Mainz 132 in the quasi-elastic (QE) kinematic regime. The asymmetry is a superposition of various distinct contributions and described by nuclear form factors [133] which were taken from other experiments. Scattering off carbon at even lower energies needs only two elastic form factors, $G_{E}^{T=0}$ and $G_{E}^{S}$, the isoscalar electromagnetic and strange quark electric form factors. The dependence on $G_{E}^{T=0}$ cancels in the asymmetry [134] of the form [135136],

$$
\frac{\sigma_{R}-\sigma_{L}}{\sigma_{R}+\sigma_{L}}=\frac{3 G_{F} Q^{2}}{2 \sqrt{2} \pi \alpha}\left[\left(C_{1 u}+C_{1 d}\right)+\frac{G_{E}^{S}}{4 G_{E}^{T=0}}\right],
$$

which has been measured in elastic $e$-C scattering [137] at the MIT-Bates accelerator. Measurements of PV elastic $e-p$ and QE $e$-D scattering [138] at the same facility yielded a value for $C_{2 u}-C_{2 d}$. However, there is uncertainty in the SM prediction due to the presence of the proton anapole moment [139 140].

Table 6] summarizes the lepton-hadron scattering experiments described above. We applied corrections for $\alpha\left(Q^{2}\right) \neq \alpha$. The most precise results from APV (discussed in the next Subsection) are also shown. Furthermore, the weak charge of the proton,

$$
Q_{W}(p)=2 C_{1 u}+C_{1 d}
$$

will be measured at the Jefferson Lab [17] in elastic e-p scattering. Ward identities associated with the weak NC protect $Q_{W}(p)$ (defined at $Q^{2}=0$ ) from incalculable strong interaction effects and 
$Q_{W}(p)$ can be computed with a high degree of reliability 142. Approximately linear effects due to $Q^{2} \approx 0.03 \mathrm{GeV}^{2} \neq 0$ can be accounted for experimentally by extrapolating across various $Q^{2}$ points performed by other experiments (see Section 6.21). A new and more precise deep inelastic $e$-D scattering experiment at SLAC has also been suggested [143] conceivably including an electron charge asymmetry as a separate observable.

We performed a simultaneous fit to the couplings defined in Eq. (35) in addition to the combination $\tilde{\epsilon} \equiv 2 C_{3 u}-C_{3 d}$, including all theoretical and experimental uncertainties and correlations. The result is,

$$
\begin{array}{llll}
\tilde{\alpha}=-0.67 & \pm 0.10 & (\mathrm{SM}=-0.530) \\
\tilde{\beta}=+0.007 & \pm 0.040 & (\mathrm{SM}=-0.062) \\
\tilde{\gamma}=+0.144 & \pm 0.006 & (\mathrm{SM}=+0.153) \\
\tilde{\delta}=+1.4 & \pm 1.5 & (\mathrm{SM}=-0.009) \\
\tilde{\epsilon}=+0.87 & \pm 0.88 & (\mathrm{SM}=+1.500)
\end{array}
$$

with the correlation matrix ${ }^{9}$ given by:

\begin{tabular}{|c|rrrrr|}
\hline & $\tilde{\alpha}$ & $\tilde{\beta}$ & $\tilde{\gamma}$ & $\tilde{\delta}$ & $\tilde{\epsilon}$ \\
\hline$\tilde{\alpha}$ & 1.00 & -0.09 & 0.98 & -0.92 & 0.81 \\
$\tilde{\beta}$ & -0.09 & 1.00 & -0.09 & -0.02 & -0.05 \\
$\tilde{\gamma}$ & 0.98 & -0.09 & 1.00 & -0.90 & 0.80 \\
$\tilde{\delta}$ & -0.92 & -0.02 & -0.90 & 1.00 & -0.88 \\
$\tilde{\epsilon}$ & 0.81 & -0.05 & 0.80 & -0.88 & 1.00 \\
\hline
\end{tabular}

This concludes the discussion of PV charged lepton scattering off hadrons. Another possibility is to have leptons in both the initial and final states. The first PV experiment of this type is being performed at SLAC. The E158 Collaboration [16] measures the weak charge of the electron, $Q_{W}(e)$, from the left-right asymmetry in polarized Møller scattering, $e^{-} e^{-} \rightarrow e^{-} e^{-}$. A precision of better than \pm 0.001 in $\sin ^{2} \theta_{W}$ at $Q^{2} \sim 0.03 \mathrm{GeV}^{2}$ is anticipated. The result of the first of three runs yields for the mixing angle in the $\overline{\mathrm{MS}}$ renormalization scheme at the $Z$ scale, $\sin ^{2} \hat{\theta}_{W}\left(M_{Z}\right)=0.2279 \pm 0.0032$.

Since the SM tree level expressions for $Q_{W}(e)$ and $Q_{W}(p)$ are suppressed by a factor $1-4 \sin ^{2} \theta_{W} \ll 1$, these observables are particularly useful to search for a variety of NP scenarios [142144].

\subsection{Parity Violation in Atoms}

The experimental study of PV in atoms generally follows one of two approaches. The first is to measure the rotation of the polarization plane of linearly polarized light as it passes through a vapor of atoms. Such a rotation occurs because PV interactions between the atomic electrons and the nucleus induce a difference in the absorption cross section for the left- and right-handed components of the polarized light. A second method involves applying an external electric field to a vapor, thereby inducing Starkmixing of the atomic levels and leading to parity forbidden atomic transitions. The PV electron-nucleus interactions lead to small modulations of the Stark induced transitions, and their effect may be isolated by the appropriate combination of field reversals. The most precise measurement of an APV effect has been performed by the Boulder group exploiting the Stark interference method and a beam of cesium atoms [13]. A summary of APV results obtained by either of these methods appears in Table 7.

Theoretically, APV effects are described by the effective, PV atomic Hamiltonian,

$$
\hat{H}_{\mathrm{PV}}^{\text {atom }}=\hat{H}_{\mathrm{PV}}^{\text {atom }}(\mathrm{NSID})+\hat{H}_{\mathrm{PV}}^{\text {atom }}(\mathrm{NSD}),
$$

\footnotetext{
${ }^{9}$ The individual $C_{1 q}$ and $C_{2 q}$ are even stronger correlated than the combinations chosen here.
} 
Table 7: Representative results of various APV experiments. Quoted atomic theory uncertainties for the first eight entries are taken from Ref. [145]. The final entry for francium indicates a measurement only of the nuclear anapole moment.

\begin{tabular}{|c|c|c|c|c|}
\hline Element & Method & Group & $\begin{array}{l}\text { Experimental } \\
\text { Uncertainty }\end{array}$ & $\begin{array}{l}\text { Atomic Theory } \\
\text { Uncertainty }\end{array}$ \\
\hline $\mathrm{Pb}$ & spin rotation & Seattle 1983146 & $\pm 28 \%$ & $\pm 10 \%$ \\
\hline \multirow[t]{3}{*}{$\mathrm{Bi}$} & spin rotation & Seattle 1981 [147] & $\pm 18 \%$ & $\pm 15 \%$ \\
\hline & & Oxford 1991 [148] & $\pm 2 \%$ & $\pm 15 \%$ \\
\hline & & Seattle $1993 \quad 149$ & $\pm 1 \%$ & $\pm 15 \%$ \\
\hline $\mathrm{Tl}$ & spin rotation & Oxford 1991 [150] & $\pm 15 \%$ & $\pm 3 \%$ \\
\hline \multirow[t]{3}{*}{$\mathrm{Tl}$} & Stark interference & Berkeley 1985 [151 & $\pm 28 \%$ & $\pm 6 \%$ \\
\hline & & Oxford 1995 [152] & $\pm 3 \%$ & $\pm 6 \%$ \\
\hline & & Seattle 1995 [153 & $\pm 1 \%$ & $\pm 6 \%$ \\
\hline \multirow[t]{4}{*}{ Cs } & Stark interference & Boulder 1985 [154 & $\pm 12 \%$ & $\pm 1 \%$ \\
\hline & & Paris 1986 [155 & $\pm 12 \%$ & $\pm 1 \%$ \\
\hline & & Boulder 1988 [156] & $\pm 2 \%$ & $\pm 1 \%$ \\
\hline & & Boulder 1998 [13] & $\pm 0.35 \%$ & $\pm 0.5 \%$ \\
\hline $\mathrm{Yb}$ & isotope ratios $\left(\mathcal{R}_{1}\right)$ & Berkeley (in progress) 157 159 & $\pm 0.1 \%$ & \\
\hline $\mathrm{Ba}^{+}$ & ion trap & Seattle (in progress) [160] & $\sim \pm 0.35 \%$ & $\lesssim \pm 1 \%$ \\
\hline Fr & atom trap & Stony Brook (in progress) 161 & $\lesssim \pm 10 \%$ & $\sim \pm 1 \%$ \\
\hline
\end{tabular}

where the second term depends on the nuclear spin (NS) while the first term is nuclear spin independent. The former is given by,

$$
\hat{H}_{\mathrm{PV}}^{\text {atom }}(\mathrm{NSID})=\frac{G_{F}}{2 \sqrt{2}} \int d^{3} x \hat{\psi}_{e}^{\dagger}(\vec{x}) \gamma_{5} \psi_{e}(\vec{x}) \rho^{\mathrm{NC}}(\vec{x})+\cdots,
$$

where $\rho^{\mathrm{NC}}(\vec{x})$ is the nuclear matrix element of the NC charge operator and where the dots indicate contributions from the spatial components of the nuclear NC. The analogous form for $\hat{H}_{\mathrm{PV}}^{\text {atom }}(\mathrm{NSD})$ is,

$$
\hat{H}_{\mathrm{PV}}^{\text {atom }}(\mathrm{NSD})=\frac{G_{F}}{\sqrt{2}} \kappa \int d^{3} x \hat{\psi}_{e}^{\dagger}(\vec{x}) \vec{\alpha} \psi_{e}(\vec{x}) \cdot \vec{I} \tilde{\rho}(\vec{x})
$$

where $\vec{\alpha}$ are Dirac matrices, $\vec{I}$ is the nuclear spin, and $\tilde{\rho}(\vec{x})$ is the nuclear density $[162$. The quantity $\kappa$ receives contributions from the hadronic axial vector $\mathrm{NC}$ as well as from the anapole moment (see below). The effect of $\hat{H}_{\mathrm{PV}}^{\text {atom }}$ is to mix atomic states of opposite parity. In cesium, the relevant mixing involves $n S_{1 / 2}$ and $n^{\prime} P_{1 / 2}$ states, with a mixing matrix element given by,

$$
\left\langle P\left|\hat{H}_{\mathrm{PV}}^{\text {atom }}(\mathrm{NSID})\right| S\right\rangle=i \frac{G_{F}}{2 \sqrt{2}} C_{S P}(Z) Q_{W}(Z, N)+\cdots
$$

where $C_{S P}$ is an atomic structure dependent coefficient that must be computed theoretically and where the dots denote small corrections arising from the spatial components of the NC, finite nuclear size, nucleon substructure, and the NSD term. Since the weak charge [cf. Eq. (40)] is given by,

$$
Q_{W}(Z, N)=(2 Z+N) C_{1 u}+(Z+2 N) C_{1 d} \approx Z\left(1-4 \sin ^{2} \theta_{W}\right)-N \approx-N
$$


the finite nuclear size corrections are dominated by the spatial dependence of the neutron density. The uncertainties associated with this correction have been estimated in Ref. 163. to be about $\pm 0.15 \%$ for cesium. The uncertainties associated with nucleon structure are comparably small [136142].

A more significant source of theoretical uncertainty arises from computations of the atomic structure dependent constants $C_{S P}(Z)$. In practical calculations, however, one must compute not only the mixing matrix elements of Eq. (46) for a tower of $P$-states that are mixed into the cesium ground state and first excited state, but also those of the electric dipole operator between the various $S$ and $P$ states as well as the energy differences appearing in the denominator in the perturbation series. Prior to the most recent measurement of cesium APV, ab initio calculations of these quantities had been carried out by the Notre Dame [164 165] and Novosibirsk [166] groups. The uncertainty in $Q_{W}(Z, N)$ associated with these computations were estimated to be about one percent. Following their $0.35 \%$ measurement of the $\mathrm{PV}$ transition in cesium [13, the members of the Boulder group performed additional measurements of transition dipole amplitudes and argued that the results considerably reduced the theoretical, atomic structure uncertainty in $Q_{W}$ for cesium, with a combined experimental and theoretical error of $0.6 \%$ [14]. With this reduced uncertainty, the measurement implied a $2.5 \sigma$ deviation from the SM prediction.

The report of this deviation stimulated considerable theoretical activity. From the standpoint of particle physics, various studies argued that it suggested the presence of a light $Z^{\prime}$ [100167168], leptoquarks [169], or RPV SUSY interactions [170]. At the same time, atomic structure theorists scrutinized previous calculations and discovered several $\mathcal{O}(1 \%)$ effects that had not been properly included. Among these effects were correlation-enhanced contributions from the Breit interaction 171172, contributions from the Uehling potential [173174, and QED vertex and self-energy corrections that are amplified in the presence of the nuclear field [175-178. Inclusion of the Uehling potential contribution tends to increase the disagreement between the experimental and SM values for $Q_{W}$, whereas the Breit correction and QED vertex and self-energy contributions reduce it. The net result is the value,

$$
Q_{W}^{\mathrm{Cs}}(\exp .)=-72.69 \pm 0.48
$$

in agreement with the SM prediction [142],

$$
Q_{W}^{\mathrm{Cs}}(\mathrm{SM})=-73.16
$$

The error in (48) includes the uncertainty from atomic structure calculations. Note that this error (obtained from the most recent atomic structure publications) has been reduced from the $1 \%$ uncertainty associated with the previous calculations in Refs. 164 166. When used to extract a value for $\sin ^{2} \theta_{W}$ at $Q^{2} \approx 0$, one obtains from (48) the result indicated in Figure 1 .

The importance of the cesium result (48) is underlined by on-going experimental work in the field. The Paris group is attempting to perform a more precise version of their earlier cesium measurement, applying the Stark induced mixing technique to a cell of cesium gas. The Seattle group has undertaken a measurement of APV with trapped $\mathrm{Ba}^{+}$ions that involves looking for frequency shifts associated with parity forbidden transitions [160]. The latter involve a study of the $6 S_{1 / 2}$ (ground state) $\rightarrow 5 D_{3 / 2}$ transition that will contain a parity forbidden component due to mixing of P-states into the ground state. Ref. [179] argued that the atomic structure computations for $\mathrm{Ba}^{+}$isotopes should achieve the same level of precision as for cesium.

At Berkeley efforts are underway to measure APV effects for different isotopes of Yb. The latter approach was motivated by the observation that a comparison of APV effects in different isotopes would eliminate the large atomic structure theory uncertainties. In particular, if one forms the quantity,

$$
\mathcal{R}_{1}=\frac{A_{\mathrm{PV}}^{\mathrm{NSID}}\left(N^{\prime}\right)-A_{\mathrm{PV}}^{\mathrm{NSID}}(N)}{A_{\mathrm{PV}}^{\mathrm{NSID}}\left(N^{\prime}\right)+A_{\mathrm{PV}}^{\mathrm{NSID}}(N)},
$$


where $A_{\mathrm{PV}}^{\mathrm{NSID}}(N)$ is a NS independent atomic PV observable, and if the atomic structure effects (governed largely by the nuclear Coulomb field) do not vary appreciably along the isotope chain, then one has,

$$
\mathcal{R}_{1}=\frac{Q_{W}\left(N^{\prime}\right)-Q_{W}(N)}{Q_{W}\left(N^{\prime}\right)+Q_{W}(N)} \approx \frac{N^{\prime}-N}{N^{\prime}+N}
$$

where the dependence on atomic structure has largely canceled from the ratio. An analogous result occurs for the ratio $\mathcal{R}_{2}=A_{\mathrm{PV}}^{\mathrm{NSID}}\left(N^{\prime}\right) / A_{\mathrm{PV}}^{\mathrm{NSID}}(N)$.

Corrections to these ratios are generated by nuclear structure since the neutron distribution [and thus, the quantity $\rho^{\mathrm{NC}}(\vec{x})$ appearing in Eq. (44)] vary along the isotope chain. At present, the theoretical uncertainties associated with this effect appear to be larger than one would like for isotope measurements to provide meaningful probes of NP [163. In principle, a new measurement of the neutron distribution in $\mathrm{Pb}$ using elastic PV electron scattering at the Jefferson Lab [180] may help to reduce the nuclear structure uncertainties associated with this and similar measurements. Moreover, the NP sensitivity of the $\mathcal{R}_{i}$ is dominated by the possible effects of NP on the proton 144, making a direct measurement with, e.g., PV $e-p$ scattering, a cleaner probe. Nonetheless, work is proceeding to carry out isotope comparisons with $\mathrm{Yb}$. A combined experimental and theoretical uncertainty of $\sim 0.2 \%$ in $\mathcal{R}_{1}(\mathrm{Yb})$ would yield a similar sensitivity to NP as the determination of $Q_{W}(p)$ planned at Jefferson Lab.

While the primary focus of atomic PV measurements has been on testing the SM via the NS independent weak charge interaction, the NS dependent contribution has also received considerable attention. The latter is dominated by the nuclear anapole moment, which gives the leading PV coupling of a photon to a nucleus. For a spin-1/2 system, the anapole interaction has the form,

$$
\mathcal{L}_{\text {anapole }}=\frac{a}{M^{2}} \bar{\psi}(x) \gamma_{\mu} \gamma_{5} \psi \partial_{\nu} F^{\mu \nu}
$$

where the coefficient, $a$, is the anapole moment. The interaction in Eq. (52) vanishes for real photons, while for virtual photons it has the same contact interaction character as the low energy $Z$ exchange amplitude. As discussed in Section 6.1, the largest contributions to $a$ arise from PV nucleon-nucleon $(N N)$ interactions, whose effects grow as $A^{2 / 3}$ in nuclei. A study of the anapole moment, then, provides a probe of the $\Delta S=0$ hadronic weak interaction in nuclei. The first non-zero determination of a nuclear anapole moment was carried out for cesium at Boulder [13, and a limit on the anapole moment of thallium has been obtained at Seattle. On-going atomic PV experiments involving $\mathrm{Yb}, \mathrm{Ba}^{+}$ions, and Fr seek to isolate the anapole effect in those nuclei (for a recent review, see Ref. [162]).

\section{Charged Current Phenomena}

\section{$3.1 \mu$ Decay}

The study of heavy lepton decays continues to provide important input into the SM and constrain various SM extensions. Indeed, the muon lifetime, $\tau_{\mu}$, remains one of the most precisely measured weak interaction observables and yields, via the Fermi constant, one of the three inputs needed to determine properties of the EW gauge sector of the SM. Taking into account QED radiative corrections up to $\mathcal{O}\left(\alpha^{2}\right)$, the lifetime and Fermi constant are related through [181,

$$
\frac{1}{\tau_{\mu}}=\frac{G_{\mu}^{2} m_{\mu}^{2}}{192 \pi^{3}} f\left(\frac{m_{e}^{2}}{m_{\mu}^{2}}\right)(1+R)\left(1+\frac{3}{5} \frac{m_{\mu}^{2}}{M_{W}^{2}}\right)
$$

where,

$$
R=\frac{\alpha}{2 \pi}\left(\frac{25}{4}-\pi^{2}\right)\left[1+\frac{\alpha}{\pi}\left(\frac{2}{3} \ln \frac{m_{\mu}}{m_{e}}-3.7\right)+\left(\frac{\alpha}{\pi}\right)^{2}\left(\frac{4}{9} \ln ^{2} \frac{m_{\mu}}{m_{e}}-2.0 \ln \frac{m_{\mu}}{m_{e}}+C\right)+\cdots\right],
$$


are the QED radiative corrections and where $f(x)=1-8 x+8 x^{3}-x^{4}-12 x^{2} \ln x$. The $\mathcal{O}(\alpha)$ contribution was first computed in Refs. [182 183]. The $\mathcal{O}\left(\alpha^{2}, \alpha^{3}\right)$ terms containing $\ln m_{\mu} / m_{e}$ terms have been obtained using renormalization group methods in Ref. 184, while the non-logarithmic $\mathcal{O}\left(\alpha^{2}\right)$ contribution was worked out in Ref. [185. The constant $C$, which describes the non-logarithmic $\mathcal{O}\left(\alpha^{3}\right)$ corrections has not been computed.

The present value for the lifetime is $\tau_{\mu}=2.197035(40) \times 10^{-6} \mathrm{~s}$, which leads to $G_{\mu}=1.16637(1) \times$ $10^{-5} \mathrm{GeV}^{-2}$ (the subscript, $\mu$, indicates a value for the Fermi constant taken from the muon lifetime). The dominant contribution to the uncertainty arises from the experimental error in $\tau_{\mu}(18 \mathrm{ppm})$, with small errors arising from the uncertainty in the neutrino mass $(10 \mathrm{ppm})$, the muon mass $(0.38 \mathrm{ppm})$ and from higher order QED contributions (0.50 ppm).

By itself, the value for $G_{\mu}$ cannot be used to constrain NP. However, requiring consistency between $G_{\mu}$ and other SM quantities can lead to constraints [181]. For example, given values for the fine structure constant, the $Z$ boson mass, and $G_{\mu}$, the SM predicts a value for the weak mixing angle as a function of all the other parameters in the theory,

$$
\hat{s}^{2} \hat{c}^{2}=\frac{\pi \alpha}{\sqrt{2} G_{\mu} M_{Z}^{2}(1-\Delta \hat{r})},
$$

where the hat indicates quantities renormalized in the $\overline{\mathrm{MS}}$ scheme, $\hat{s}^{2}=\sin ^{2} \hat{\theta}_{W}\left(\mu=M_{Z}\right)$, and $\Delta \hat{r}$ is a radiative correction parameter. Alternately, if one treats all the SM parameters (including the value of $\hat{s}^{2}$ ) as independent quantities to be taken from experiment, Eq. (55) is a self-consistency test constraining NP that could affect the value of $\Delta \hat{r}=\Delta \hat{r}^{\mathrm{SM}}+\Delta \hat{r}^{\mathrm{NEW}}$. Updating Ref. [181] we find,

$$
\Delta \hat{r}^{\mathrm{NEW}}=0 \pm 0.0006
$$

This can be used to constrain a variety of NP scenarios [181. For example, if an excited $W$ boson contributed to the decay rate, then its mass would have to satisfy,

$$
M_{W^{*}}>3.3 \sqrt{C} \frac{g^{*}}{g} \mathrm{TeV},
$$

where $C$ is a model dependent constant expected to be of $\mathcal{O}(1)$, and $g^{*}$ is the gauge coupling of the $W^{*}$. In a similar way, Eq. (55) can be used to derive constraints for SUSY scenarios [170 186] (see Refs. [187-194 for earlier analyses).

At present, the range (56) is determined by the uncertainties in the values of $m_{t}$ and $\alpha\left(M_{Z}\right)$ that appear in $\Delta \hat{r}^{\mathrm{SM}}$, and the value of $\hat{s}^{2}$ entering Eq. (55). Substantial improvements in the precision for these quantities must be achieved before the uncertainty in $G_{\mu}$ will present a serious limitation to future improvements in NP sensitivity. Nevertheless, a more precise measurement of $\tau_{\mu}$ is being carried out at PSI by the FAST [195] and $\mu$ Lan Collaborations [196]. The goal for both measurements is a 2 ps (1 ppm) uncertainty in the lifetime.

In addition to precision measurements of $\tau_{\mu}$, studies of the $\mu$ decay spectral shape and $\beta$-asymmetry provide tests of EW theory ${ }^{10}$. These properties have historically been described by the Michel parameters [198 199, which appear in the partial decay rate for a $\mu^{ \pm}$:

$$
\begin{aligned}
d \Gamma= & \frac{G_{\mu}^{2} m_{\mu}^{5}}{192 \pi^{3}} \frac{d \Omega}{4 \pi} x^{2} d x \times\left\{\frac{1+h(x)}{1+4 \eta\left(m_{e} / m_{\mu}\right)}\left[12(1-x)+\frac{4}{3} \rho(8 x-6)+24 \frac{m_{e}}{m_{\mu}} \frac{(1-x)}{x} \eta\right]\right. \\
& \left. \pm P_{\mu} \xi \cos \theta\left[4(1-x)+\frac{4}{3} \delta(8 x-6)+\frac{\alpha}{2 \pi} \frac{g(x)}{x^{2}}\right]\right\},
\end{aligned}
$$

where $x=\left|\vec{p}_{e}\right| /\left|\vec{p}_{e}\right|_{\max }, \theta=\cos ^{-1}\left(\hat{p}_{e} \cdot \hat{s}_{\mu}\right), P_{\mu}$ is the $\mu^{ \pm}$polarization, and $h(x)$ and $g(x)$ are momentum dependent radiative corrections. The SM predictions for the Michel parameters, $\rho, \delta$, $\xi$, and $\eta$, along with the present experimental limits are listed in Table 8

\footnotetext{
${ }^{10}$ Studies of the $\beta$-polarization may also lead to constraints on NP (see, e.g., Ref. [197]).
} 
Table 8: Present experimental values for the Michel parameters, compared with SM predictions. Experimental errors have been combined in quadrature. Projected uncertainties for the TWIST measurement are shown in the last column.

\begin{tabular}{|c|c|c|c|}
\hline Parameter & Present & SM & TWIST (projected) \\
\hline$\rho$ & $0.7518 \pm 0.0026[200]$ & $3 / 4$ & \pm 0.0001 \\
$\delta$ & $0.7486 \pm 0.0040[201]$ & $3 / 4$ & \pm 0.00014 \\
$P_{\mu} \xi$ & $1.0027 \pm 0.0085[202]$ & 1 & \pm 0.00013 \\
$\eta$ & $-0.007 \pm 0.013[203]$ & 0 & \pm 0.003 \\
\hline
\end{tabular}

Note that the effect of $\eta$ on the differential rate is suppressed by $m_{e} / m_{\mu}$, making this quantity more difficult to measure than the other Michel parameters. Indeed, the level of agreement of the SM predictions for the shape parameters, $\rho$ and $\delta$, compared to experiment is quite high (per mille), while the precision for $\eta$ and the asymmetry parameter $P_{\mu} \xi$ is presently a factor of ten weaker. One expects significant improvements in these limits from the TWIST Collaboration 204, which has undertaken a new measurement of polarized $\mu^{+}$decay at TRIUMF. As indicated in Table 8, the Collaboration expects to decrease the experimental errors by factors ranging from 60 for $P_{\mu} \xi$ to 4 for $\eta$.

With the expected improvement in precision, the results of the TWIST experiment could have significant implications for NP that might affect muon decay. Historically, the effects of non-SM interactions on the Michel parameters have been characterized by a general set of four-fermion contact operators,

$$
\mathcal{L}_{\mu \text { decay }}^{\mathrm{eff}}=\frac{G_{\mu}}{\sqrt{2}} \sum_{j} \bar{\psi}_{e} \Gamma_{j} \psi_{\mu} \bar{\psi}_{\nu_{\mu}} \Gamma_{j}\left(C_{j}+C_{j}^{\prime} \gamma_{5}\right) \psi_{\nu_{e}}
$$

where the sum runs over all of the independent Dirac matrices $\Gamma_{j}$. While the operators appearing in Eq. (59) are non-renormalizable, they may arise in the low energy limit of renormalizable gauge theories. In theories such as the SM that contain purely left- or right-handed gauge interactions, all but the vector and axial vector type couplings, $C_{V, A}$ and $C_{V, A}^{\prime}$, vanish. Scalar and pseudoscalar interactions can be induced, for example, in the presence of mixing between left- and right-handed gauge bosons (see below). General expressions for the Michel parameters in terms of the $C_{j}$ and $C_{j}^{\prime}$ can be found, for example, in Ref. 205].

To illustrate, we consider the effects of right-handed gauge interactions on the Michel parameters. An extensive analysis of such effects has been carried out in Ref. 206]. For a situation involving an additional, light right-handed gauge boson that mixes with the $S U(2)_{L}$ gauge boson, one has,

$$
\begin{aligned}
& W_{1}=\cos \zeta W_{L}-\sin \zeta e^{-i \omega} W_{R} \\
& W_{2}=\sin \zeta W_{L}+\cos \zeta e^{-i \omega} W_{R}
\end{aligned}
$$

for the two mass eigenstates with $M_{2}>M_{1}$. The resultant effective, low energy interaction for muon decay is given by,

$$
\mathcal{L}_{\text {eff }}^{\mu \text { decay }}=4 \sum_{i j} c_{i j} \bar{e}_{i} \gamma^{\lambda} \nu_{e i} \bar{\nu}_{\mu j} \gamma_{\lambda} \mu_{j}
$$

where the sum runs over all chiralities $i, j=L, R$ and where the neutrino flavor states may be mixtures of mass eigenstates. In terms of the couplings, masses, and mixing angles, one has [206],

$$
c_{L L}=\frac{g_{L}^{2}}{8 M_{1}^{2}} \cos ^{2} \zeta+\frac{g_{R}^{2}}{8 M_{2}^{2}} \sin ^{2} \zeta,
$$




$$
\begin{gathered}
c_{R R}=\frac{g_{L}^{2}}{8 M_{1}^{2}} \sin ^{2} \zeta+\frac{g_{R}^{2}}{8 M_{2}^{2}} \cos ^{2} \zeta, \\
c_{L R}=c_{R L}^{*}=-\frac{g_{L} g_{R}}{8 M_{1}^{2}}\left(1-\frac{M_{1}^{2}}{M_{2}^{2}}\right) \sin \zeta \cos \zeta e^{i \omega} .
\end{gathered}
$$

In order to translate these effective couplings into the Michel parameters, one must consider various scenarios for the neutrino sector. For example, assuming massive Dirac neutrinos leads to,

$$
\begin{aligned}
\rho & =\frac{3}{4} \frac{1+\left|\kappa_{R R}\right|^{2} \tilde{v}_{e} \tilde{v}_{\mu}}{1+\left|\kappa_{R R}\right|^{2} \tilde{v}_{e} \tilde{v}_{\mu}+\left|\kappa_{R L}\right|^{2}\left(\tilde{v}_{\mu}+\tilde{v}_{e}\right)} \\
\eta & =0 \\
\xi & =\frac{1-\left|\kappa_{R R}\right|^{2} \tilde{v}_{e} \tilde{v}_{\mu}}{1+\left|\kappa_{R R}\right|^{2} \tilde{v}_{e} \tilde{v}_{\mu}+\left|\kappa_{R L}\right|^{2}\left(\tilde{v}_{\mu}+\tilde{v}_{e}\right)}, \\
\delta & =\frac{1-\left|\kappa_{R R}\right|^{2} \tilde{v}_{e} \tilde{v}_{\mu}}{1-\left|\kappa_{R R}\right|^{2} \tilde{v}_{e} \tilde{v}_{\mu}+3\left|\kappa_{R L}\right|^{2}\left(\tilde{v}_{\mu}-\tilde{v}_{e}\right)},
\end{aligned}
$$

for the Michel parameters and,

$$
P_{\mu}=\frac{\left|1-\kappa_{L R} \lambda e^{i \alpha}\right|^{2}-\left|\kappa_{R L}-\kappa_{R R} \lambda e^{i \alpha}\right|^{2} \tilde{v}_{\mu}}{\left|1-\kappa_{L R} \lambda e^{i \alpha}\right|^{2}+\left|\kappa_{R L}-\kappa_{R R} \lambda e^{i \alpha}\right|^{2} \tilde{v}_{\mu}},
$$

for the polarization. The quantities $\tilde{v}_{\ell}$ are ratios of sums over the mixing angles for the right- and left-handed neutrinos,

$$
\tilde{v}=\frac{\sum_{i}^{\prime}\left|V_{l i}\right|^{2}}{\sum_{i}^{\prime}\left|U_{l i}\right|^{2}}
$$

with the $U_{l i}\left(V_{l i}\right)$ being the analogs of the CKM matrix for left-handed (right-handed) Dirac neutrinos and the prime indicating that only mass eigenstates produced in the decay are included, $\lambda=$ $\cos \theta_{1}^{R} / \cos \theta_{1}^{L}$ is the ratio of $(1,1)$ entries in the right- and left-handed CKM matrices, $\alpha$ is the CP violating phase in the right-handed CKM matrix, and $\kappa_{i j}=c_{i j} / c_{L L}$.

As Eqs. (63) 68) make evident, the Michel parameters depend in a complicated way on the couplings, mixing angles, and masses that appear in the simplest, but most general, left-right symmetric model with massive Dirac neutrinos. Allowing for Majorana mass terms introduces additional contributions to expression for the Michel spectrum. Thus, the analysis of the Michel spectrum must occur in the context of a more general study of CC processes, including direct searches for a $W_{R}$ in collider experiments, light quark $\beta$ decay, neutrino oscillations, neutrinoless $\beta \beta$ decay, etc. Such a comprehensive study has yet to be performed.

Some simplifications occur by considering the combination of Michel parameters

$$
R=1-\frac{\delta \xi}{\rho} P_{\mu}
$$

As a practical matter, only the combination $\xi P_{\mu}$ can be accessed by experiment, making $R$ an appropriate quantity to constrain experimentally. Expanding the foregoing expressions for the Michel parameters and $P_{\mu}$ to second order in small quantities, one obtains [206],

$$
R \approx 2 t^{2} \tilde{v}_{e} \tilde{v}_{\mu}+2 t_{\theta}^{2} \tilde{v}_{\mu}+2 \zeta_{g}^{2} \tilde{v}_{\mu}+4 t_{\theta} \zeta_{g} \tilde{v}_{\mu} \cos \left(\alpha_{+} \omega\right)
$$

where $t=\left(g_{R} / g_{L}\right)^{2}\left(M_{1} / M_{2}\right)^{2}, t_{\theta}=t \lambda$, and $\zeta_{g}=\left(g_{R} / g_{L}\right) \zeta$. Information on the combination of phases $\alpha+\omega$ can be derived from searches for the electric dipole moment of the electron, neutron, or neutral atoms, which are sensitive to the combination $\zeta_{g} \lambda \sin (\alpha+\omega)$, while neutron, pion, and nuclear $\beta$ decays provide independent constraints on $\zeta_{g}$. Studies of neutrino properties are clearly required for $\tilde{v}_{\ell}$. Collider experiments presently provide upper bounds on the mass ratios $M_{1}^{2} / M_{2}^{2}$, though the extraction of these bounds depends to some degree on assumptions about the right-handed CKM matrix and the relative strengths of the left- and right-handed couplings. 


\subsection{Pion Decay}

The decay modes of the $\pi^{ \pm}$have long been a subject of study in EW physics. The dominant decay mode, $\pi^{-} \rightarrow \mu^{-} \bar{\nu}_{\mu}$ provides a value for the pion decay constant, $F_{\pi}$, that encodes the effects of nonperturbative strong interactions involving the light quarks in the decay. Since these effects cannot be computed at present with high precision, the dominant decay mode does not provide a useful testing ground for the SM EW interaction. However, the value of $F_{\pi}$ obtained from this decay plays an important role in the analysis of chiral dynamics in strong interactions. In contrast, a comparison of the rates $\Gamma\left[\pi^{+} \rightarrow \mu^{-} \bar{\nu}_{\mu}(\gamma)\right]$ and $\Gamma\left[\pi^{-} \rightarrow e^{-} \bar{\nu}_{e}(\gamma)\right]$ is insensitive to $F_{\pi}$ at leading order and can be used to study the underlying EW interaction. Similarly, the pion $\beta$ decays $\pi^{+} \rightarrow \pi^{0} e^{+} \nu_{e}\left(\pi^{-} \rightarrow \pi^{0} e^{-} \bar{\nu}_{e}\right)$ and their radiative counterparts are also quite insensitive to strong interaction uncertainties, making them in principle an interesting SM testing ground.

When extracting the value of $F_{\pi}$ from $\Gamma\left[\pi^{-} \rightarrow \mu^{-} \bar{\nu}_{\mu}(\gamma)\right]$, one must take into consideration EW radiative corrections. Doing so ensures that they are not inadvertently included (via $F_{\pi}$ ) in strong interaction processes such as $\pi-N$ scattering. In contrast to the situation with muon decay, however, the treatment of these radiative corrections is convention dependent and entails some degree of theoretical uncertainty. These features arise because some of the $\mathcal{O}(\alpha)$ contributions involve loops containing light quarks that interact non-perturbatively. The most widely used convention for treating the radiative corrections has been given by Marciano and Sirlin [207]. Including all effects through order $G_{\mu}^{2} \alpha$ yields,

$$
\begin{array}{r}
\Gamma\left[\pi^{-} \rightarrow \ell^{-} \bar{\nu}_{\ell}(\gamma)\right]=\frac{G_{\mu}^{2}\left|V_{u d}\right|^{2}}{4 \pi} F_{\pi}^{2} m_{\pi} m_{\ell}^{2}\left[1-\frac{m_{\ell}^{2}}{m_{\pi}^{2}}\right]\left[1+\frac{2 \alpha}{\pi} \ln \frac{M_{Z}}{\mu}\right] \\
\times\left[1-\frac{\alpha}{\pi}\left\{\frac{3}{2} \ln \frac{\mu}{m_{\pi}}+\bar{C}_{1}(\mu)+\bar{C}_{2}(\mu) \frac{m_{\ell}^{2}}{\Lambda_{\chi}^{2}} \ln \frac{\mu^{2}}{m_{\ell}^{2}}+\bar{C}_{3}(\mu) \frac{m_{\ell}^{2}}{\Lambda_{\chi}^{2}}+\cdots\right\}\right]\left[1+\frac{\alpha}{\pi} F(x)\right],
\end{array}
$$

where $\bar{C}_{i}$ are a priori unknown constants that parameterize presently incalculable non-perturbative QCD effects, $\Lambda_{\chi}=4 \pi F_{\pi}$ is the chiral scale, the dots denote terms suppressed by additional powers of the lepton mass square, $m_{\ell}^{2} / \Lambda_{\chi}^{2}$, and $x=m_{\ell}^{2} / m_{\pi}^{2}$.

The function $F(x)$, along with the terms containing the $\bar{C}_{i}$, arise from QED corrections to the decay of a point-like pion. The first $\mathcal{O}(\alpha)$ correction containing the $\ln M_{Z}$ is a short-distance contribution. Symmetry considerations protect part of this term from receiving any perturbative corrections, while another component receives corrections. In addition, Ref. 207] summed the contributions of the form $\left[(\alpha / \pi) \ln \left(M_{Z} / \mu\right)\right]^{n}$ for all $n$, using the RG to produce an improved estimate of the short distance correction factor, $S_{E W}\left(\mu, M_{Z}\right)$ that replaces $1+2(\alpha / \pi) \ln \left(M_{Z} / \mu\right)$. Choosing $\mu=m_{\rho}$, one has $S_{E W}\left(\mu, M_{Z}\right)=1.0232$.

More serious theoretical uncertainties arise from the terms proportional to the $\bar{C}_{i}$. In general, they depend on the choice of scale associated with matching short- and long-distance contributions ${ }^{11}$. In particular, the $\mu$ dependence of $\bar{C}_{1}(\mu)$ must cancel the $\mu$ dependence of the short-distance, RG-improved correction factor. The authors have estimated the uncertainty in $\bar{C}_{1}$ by varying $\mu$ from $m_{\rho}$ by a factor of two and requiring a corresponding variation in $\bar{C}_{1}(\mu)$. Taking $\bar{C}_{1}\left(m_{\rho}\right)=0$ they estimate $\delta \bar{C}_{1}= \pm 2.4$, corresponding to a $\pm 0.56 \%$ correction to the rate.

An estimate for $\bar{C}_{2}$ can be obtained using PCAC and the ratio of axial and vector form factors in radiative pion decay. The uncertainty associated with $\bar{C}_{3}$ should be small as its magnitude is $(\alpha / \pi)\left(m_{\mu}^{2} / \Lambda_{\chi}^{2}\right) \bar{C}_{3} \approx 1.9 \times 10^{-5} \bar{C}_{3}$. Including these effects, using the latest value for the lifetime and branching ratio [113,

$$
\tau_{\pi^{ \pm}}=(2.6033 \pm 0.0005) \times 10^{-8} s, \quad \frac{\Gamma\left(\pi^{+} \rightarrow \mu^{+} \nu_{\mu}\right)}{\Gamma_{\text {tot }}}=(99.98770 \pm 0.00004) \%,
$$

\footnotetext{
${ }^{11}$ Our conventions differ slightly from those of Ref. [207], since we have normalized the terms containing powers of $m_{\ell}^{2}$ to $\Lambda_{\chi}^{2}$ rather than to $m_{\rho}^{2}$.
} 
and the values of $G_{\mu}$ and $\left|V_{u d}\right|$ from muon and super-allowed, nuclear $\beta$ decays (see below), one obtains,

$$
F_{\pi}=92.4 \pm 0.07 \pm 0.25 \mathrm{MeV}
$$

where the first error is from the experimental uncertainty in $\left|V_{u d}\right|$ and the second is associated with $\bar{C}_{1}$.

In contrast to $\Gamma\left[\pi^{-} \rightarrow \ell^{-} \bar{\nu}_{\ell}(\gamma)\right]$, the ratio $R_{e / \mu}$ of electronic to muonic widths is fairly insensitive to strong interaction uncertainties and can provide an interpretable test of the electron-muon universality of the SM. In particular,

$$
R_{e / \mu}=\frac{\Gamma\left[\pi^{-} \rightarrow e^{-} \bar{\nu}_{e}(\gamma)\right]}{\Gamma\left[\pi^{-} \rightarrow \mu^{-} \bar{\nu}_{\mu}(\gamma)\right]}=\frac{m_{e}^{2}}{m_{\mu}^{2}}\left[\frac{m_{\pi}^{2}-m_{e}^{2}}{m_{\pi}^{2}-m_{\mu}^{2}}\right]^{2}\left\{1+\frac{\alpha}{\pi}\left[F\left(\frac{m_{e}}{m_{\pi}}\right)-F\left(\frac{m_{\mu}}{m_{\pi}}\right)+\frac{m_{\mu}^{2}}{\Lambda_{\chi}^{2}}\left(\bar{C}_{2} \ln \frac{m_{\mu}^{2}}{\Lambda_{\chi}^{2}}+\bar{C}_{3}\right)\right]\right\},(
$$

where terms proportional to $\alpha m_{e}^{2}$ are negligible and have been dropped. After including structure dependent bremsstrahlung corrections and re-summing terms of the form $\left[\alpha / \pi \ln \left(m_{e} / m_{\mu}\right)\right]^{n}$ that arise in the difference $F\left(m_{e} / m_{\pi}\right)-F\left(m_{\mu} / m_{\pi}\right)$ one obtains [207],

$$
R_{e / \mu}^{\mathrm{SM}}=(1.2352 \pm 0.0005) \times 10^{-4}
$$

where the error originates dominantly from the structure dependent bremsstrahlung contributions.

Precise determinations of $R_{e / \mu}$ have been carried out at PSI 208] and TRIUMF [209], averaging to,

$$
\frac{R_{e / \mu}^{\exp }}{R_{e / \mu}^{\mathrm{SM}}}=0.9966 \pm 0.0030 \pm 0.0004
$$

where the first error is experimental and the second is the estimated theoretical uncertainty. At present, one has no indication of any disagreement with the SM, though the ratio (77) does provide rather stringent constraints on possible universality violating, NP contributions. For example, the exchange of squarks $(\tilde{q})$ in RPV SUSY may lead to a non-universal contribution [170 210],

$$
\frac{R_{e / \mu}}{R_{e / \mu}^{\mathrm{SM}}}=1+2\left[\Delta_{11 k}^{\prime}\left(\tilde{d}_{R}^{k}\right)-\Delta_{21 k}^{\prime}\left(\tilde{q}_{R}^{k}\right)\right],
$$

where $\Delta_{11 k}^{\prime}\left(\tilde{d}_{R}^{k}\right)=\left|\lambda_{11 k}^{\prime}\right|^{2} / 4 \sqrt{2} G_{F} M_{\tilde{d}_{R}^{k}}^{2}$, etc., with $\lambda_{i j k}^{\prime}$ denoting the RPV coupling of various generations of squarks with masses $M_{\tilde{q}_{k}}$. The experimental agreement with the SM prediction illustrated by the ratio (177) places substantial constraints on the possible size of RPV effects in other low energy weak processes that also depend on the $\Delta_{i j k}^{\prime}$ 98170.

In addition, $\mathrm{CC}$ universality tests can be performed by the study of pion $\beta$ decays. In this case, the transition involves hadronic matrix elements of the charged vector current, so the theoretical prediction for the rate is protected from large and theoretically uncertain strong interaction corrections of the type encoded by $F_{\pi}$. However, some uncertainties do appear at $\mathcal{O}(\alpha)$ due to the diagrams of the type in Figure 4. The benchmark study of the SM, EW radiative corrections to pion $\beta$ decay was performed by Sirlin [211], who used current algebra techniques. A more recent analysis within the context of chiral perturbation theory $(\chi \mathrm{PT})$ has been carried out by Cirigliano et al. [212]. The latter analysis also includes the corrections due to pion form factors that were not included in the earlier studies.

Experimentally, the study of pion $\beta$ decay is a challenging enterprise, given that the branching ratio for this decay mode is of the order $\sim 10^{-8}$. The most accurate result has been published in Ref. [213], leading to the Particle Data Group value [113,

$$
\mathcal{B}\left(\pi^{+} \rightarrow \pi^{0} e^{+} \nu_{e}\right)=(1.025 \pm 0.034) \times 10^{-8} .
$$

After inclusion of EW radiative corrections [212, one can extract,

$$
\left|V_{u d}\right|^{2}=0.9675 \pm 0.0160 \pm 0.0005
$$




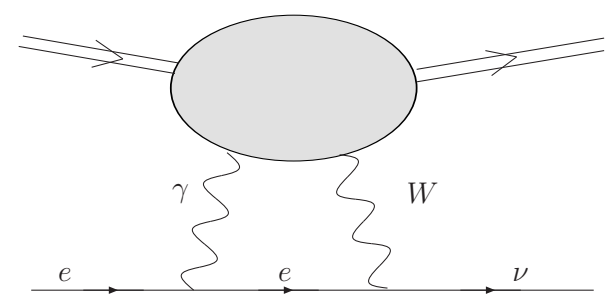

Figure 4: The $\mathcal{O}(\alpha)$ box graph correction to pion, neutron, and nuclear $\beta$ decay generated by the exchange of one $W$ boson and one $\gamma$. The double lines indicate initial and final hadronic states, respectively.

where the first error is experimental and the second theoretical. The latter is dominated by extrapolation of the pion form factor $f_{+}$to $Q^{2}=0$ and is negligible compared to the experimental uncertainty.

A reduction in the experimental error bar is a primary goal of the PIBETA experiment [214] underway at PSI. The experiment relies on a $113 \mathrm{MeV}$ beam of $\pi^{+}$stopped in a plastic scintillator target. Energy from the decay products is deposited in a CsI shower calorimeter. The ultimate goal of the measurement is to determine $\mathcal{B}\left(\pi^{+} \rightarrow \pi^{0} e^{+} \nu_{e}\right)$ to $0.5 \%$ precision, representing a factor of 7 improvement over the present world average. A new result with $0.8 \%$ uncertainty, based on a partial data set, has recently been reported by the Collaboration [215].

The PIBETA Collaboration is also carrying out a study of radiative pion decay (RPD). The branching ratio, $\mathcal{B}\left(\pi^{+} \rightarrow e^{+} \nu_{e} \gamma\right)$, is sensitive to the ratio of axial vector and vector pion form factors, $F_{A}$ and $F_{V}$, respectively, defined as [216217],

$$
\begin{aligned}
\left\langle\gamma(q, \varepsilon)\left|\bar{d} \gamma_{\lambda} u\right| \pi^{+}(p)\right\rangle & =e F_{V} \epsilon_{\mu \lambda \rho \sigma} \varepsilon^{\mu} \frac{p^{\rho} q^{\sigma}}{m_{\pi}} \\
\left\langle\gamma(q, \varepsilon)\left|\bar{d} \gamma_{\lambda} \gamma_{5} u\right| \pi^{+}(p)\right\rangle & =i e F_{A} \varepsilon^{\mu} \frac{p \cdot q g_{\mu \lambda}-p_{\mu} q_{\lambda}}{m_{\pi}},
\end{aligned}
$$

where $p$ is the pion momentum, and $q$ and $\varepsilon$ are the photon momentum and polarization, respectively. Present constraints on RPD give [113], $F_{V}=0.0259 \pm 0.0005$, and $F_{A} \approx 0.5 F_{V}$. Recently, the PIBETA Collaboration has performed a precise determination of the ratio, $\gamma=F_{A} / F_{V}$, yielding [218],

$$
\gamma=0.443 \pm 0.014
$$

The presence of only these two form factors $\left(F_{V, A}\right)$ implies a spectral shape for the RPD as a function of $\left(2 E_{e^{+}} / m_{\pi^{+}}\right) \sin ^{2}\left(\theta_{e \gamma} / 2\right)$. An analysis of the PIBETA data set indicates a departure from this expectation for hard photons and soft positrons [218. If one ultimately does not find a refined SM prediction that is consistent with the observed spectral shape, the explanation could lie in the presence of a non-vanishing tensor form factor [217] arising from a lepton-quark interaction producing an amplitude,

$$
\mathcal{M}\left(\pi^{+} \rightarrow e^{+} \nu_{e} \gamma\right)=\frac{G_{\mu}\left|V_{u d}\right|}{\sqrt{2}} i e F_{T} \varepsilon^{\lambda} q^{\mu} \bar{e} \sigma_{\lambda \mu}\left(1-\gamma_{5}\right) \nu_{e}
$$

Including such a non-vanishing tensor amplitude with $F_{T} \approx 0.0016$ appears to produce better agreement with the preliminary experimental PIBETA spectrum [214. While the value of $F_{T}$ vanishes at tree level in the SM, one can presently not exclude an induced $F_{T}$ arising from radiative corrections. Alternatively, this type of term may arise in various leptoquark scenarios or from sfermion exchange in RPV SUSY. 


\subsection{Beta Decay, Kaon Decay, and CKM Unitarity}

As illustrated in the previous Subsection, one of the arenas in which the predictions of the SM have been tested most precisely at low energies is in the weak decays of light quarks. Of particular interest is the first row of the CKM matrix and the SM unitarity requirement,

$$
\left|V_{u d}\right|^{2}+\left|V_{u s}\right|^{2}+\left|V_{u b}\right|^{2}=1
$$

The largest contributor to the unitarity sum is $\left|V_{u d}\right|$, which can be determined from the $\beta$ decay of the pion, neutron, or nuclei. The current experimental work on pion $\beta$ decay has been discussed above, so we concentrate here on neutron and nuclear $\beta$ decays. In both cases, the relevant quantity that characterizes the total decay rate is the so-called $f t$ value,

$$
f t=\frac{K}{\left(G_{F}^{\beta}\right)^{2}\left\langle M_{V}\right\rangle^{2}+\left(G_{A}^{\beta}\right)^{2}\left\langle M_{A}\right\rangle^{2}},
$$

where $t$ is the half-life, $f$ is a factor that corrects for the outgoing $\beta$ particle wave-function, $G_{A}=$ $G_{\mu} V_{u d} g_{A}$ with $g_{A}$ being the axial vector coupling of the nucleon to the $W^{ \pm}$boson, $M_{V}\left(M_{A}\right)$ are vector (axial vector) matrix elements, and $K$ is the combination of constants,

$$
K=\hbar\left(2 \pi^{3} \ln 2\right)(\hbar c)^{6} /\left(m_{e} c^{2}\right)^{5} .
$$

The quantity $G_{F}^{\beta}$ can be expressed in terms of $G_{\mu}, V_{u d}$, and radiative corrections to the $\beta$ decay $\left(\Delta r_{\beta}\right)$ and $\mu$ decay $\left(\Delta r_{\mu}\right)$ amplitudes,

$$
\frac{G_{F}^{\beta}}{G_{\mu}}=V_{u d}\left(1+\Delta r_{\beta}-\Delta r_{\mu}\right)
$$

The quantity $\Delta r_{\beta}$ is sensitive to various non-perturbative strong interaction effects that contribute to the EW radiative corrections. The matrix elements $M_{V, A}$ are similarly dependent on hadronic structure. For neutron decay, for example, one has $\left\langle M_{V}\right\rangle^{2}=1$ and $\left\langle M_{A}\right\rangle^{2}=3$.

The neutron lifetime, $\tau_{n}$, depends on both $G_{F}^{\beta}$ and $G_{A}^{\beta}$ and both of these quantities are proportional to $V_{u d}$. Unlike $G_{F}^{\beta}$, which arises from the vector coupling of the $W^{ \pm}$boson to light quarks, $G_{A}^{\beta}$ arises from the axial vector charge changing quark current and, thus, is susceptible to significant strong interaction renormalization. The quantity $g_{A}=-1.2670 \pm 0.0030$ encodes this renormalization. At present, one cannot compute it directly from QCD with the precision needed to perform a significant determination of $V_{u d}$ from $\tau_{n}$. Instead, an experimental separation of $G_{F}^{\beta}$ and $G_{A}^{\beta}$ is needed. In practice, such a separation can be obtained by combining a determination of $\tau_{n}$ with a measurement of any one of several $\beta$ decay correlation coefficients. The latter appear in the partial rate [219],

$$
d \Gamma \propto N\left(E_{e}\right)\left[1+a \frac{\vec{p}_{e} \cdot \vec{p}_{\nu}}{E_{e} E_{\nu}}+b \frac{m_{e}}{E_{e}}+A \frac{\vec{p}_{e} \cdot\left\langle\vec{\sigma}_{n}\right\rangle}{E_{e}}+B \frac{\vec{p}_{\nu} \cdot\left\langle\vec{\sigma}_{n}\right\rangle}{E_{\nu}}+D\left\langle\vec{\sigma}_{n}\right\rangle \frac{\vec{p}_{e} \times \vec{p}_{\nu}}{E_{e} E_{\nu}}\right] d \Omega_{e} d \Omega_{\nu} d E_{e}
$$

where $N\left(E_{e}\right)=p_{e} E_{e}\left(E_{0}-E_{e}\right)^{2}, E_{e}\left(E_{\nu}\right)$ and $\vec{p}_{e}\left(\vec{p}_{\nu}\right)$ are the $\beta$ (neutrino) energy and momentum, respectively, and $\vec{\sigma}_{n}$ is the neutron polarization. The coefficients $a, A$, and $B$ can be expressed in terms of the ratio, $\lambda=G_{A}^{\beta} / G_{F}^{\beta}$, as,

$$
a=\frac{1-\lambda^{2}}{1+3 \lambda^{2}}, \quad A=-2 \frac{\lambda(1+\lambda)}{1+3 \lambda^{2}}, \quad B=2 \frac{\lambda(\lambda-1)}{1+3 \lambda^{2}} .
$$

The quantity $b$ appearing in the so-called Fierz interference term is zero for purely vector and axial vector interactions, while the triple correlation parameterized by $D$ is time-reversal odd. A non-zero value for the latter can be induced by electromagnetic final state interactions between the outgoing $\beta$ particle and the proton or by possible new T-odd, parity conserving interactions. A search for the 
latter via a measurement of the $D$ coefficient has been completed by the emiT Collaboration [220], who obtained the null result,

$$
D=[-0.6 \pm 1.2 \text { (stat.) } \pm 0.5 \text { (syst.) }] \times 10^{-3} .
$$

The neutron lifetime has been obtained from both neutron beam experiments and stored ultracold neutrons (UCNs). An average of the five most recent experiments yields the current PDG value, $\tau_{n}=885.7 \pm 0.7 \mathrm{~s}$. A more precise measurement of $\tau_{n}$ is being pursued at NIST using magnetically trapped ultra-cold neutrons [221]. Historically, determinations of $\lambda$ from correlation measurements have proved more challenging due to a host of systematic effects. The quoted uncertainty for each of the four most recent cold neutron measurements of $A$ is roughly one percent. However, the central values form the different measurements are in rather poor agreement, leading to the PDG average, $A=-0.1162 \pm 0.0013$. When combined with the current uncertainty in $\tau_{n}$, this average implies a sizable uncertainty in $V_{u d}$ obtained from neutron decay.

Recent technological advances involving cold and ultra-cold neutron methods should lead to improved determinations of $\lambda$. The PERKEO Collaboration has performed a determination of $A$ with $\sim 0.6 \%$ uncertainty using cold neutrons at the ILL reactor, leading to $\lambda=-1.2739 \pm 0.0019[222$. In contrast to previous determinations of $A$, which entailed application of large, $\mathcal{O}(25 \%)$, corrections to the raw data, the subtractions applied in the latest experiment are roughly ten times smaller than the quoted uncertainty. When combined with the current world average for $\tau_{n}$, the result implies $\left|V_{u d}\right|=0.9713 \pm 0.0013$. Using the current PDG values for $\left|V_{u s}\right|$ (see below) and $\left|V_{u b}\right|$ one obtains,

$$
\left|V_{u d}\right|^{2}+\left|V_{u s}\right|^{2}+\left|V_{u b}\right|^{2}=0.9917 \pm 0.0028
$$

indicating a deviation from unitarity by more than three standard deviations.

A new measurement of $A$ presently underway at LANSCE seeks to reduce the uncertainty in $\lambda$ to the $0.1 \%$ level 223 . The experiment relies on a solid $\mathrm{D}_{2}$ moderator and the LANSCE beam of cold neutrons to create a source of ultra cold, polarized neutrons. Cold neutrons $(T<100 \mathrm{~K})$ are first produced using conventional cold moderators and subsequently cooled to $T<4 \mathrm{mK}$ via phonon interactions in $\mathrm{D}_{2}$. The experiment has thus far produced the world's record density of stored ultra cold neutrons $\left(140 \mathrm{UCN} / \mathrm{cm}^{3}\right)$. The advantage of UCNs for a measurement of $A$ is two-fold: (a) the neutrons can be stored in bottles for a duration comparable to $\tau_{n}$ and (b) a polarization of $P \sim 100 \%$ can be achieved.

A variety of future possibilities for both more precise $\tau_{n}$ determinations as well as correlation coefficient measurements have been discussed. The use of magnetically trapped UCNs in super-fluid ${ }^{4} \mathrm{He}$ has been demonstrated at NIST, and a $\tau_{n}$ measurement using this method with $\pm 1 \mathrm{~s}$ uncertainty could be carried out. The construction of an UCN beam line for fundamental neutron physics at the Spallation Neutron Source (SNS) at Oak Ridge National Laboratory would provide the capability for further improvements in precision. The present uncertainty in $\tau_{n}$ contributes an error of $\sim 0.05 \%$ to the value of $\left|V_{u d}\right|$ obtained from neutron decay, and as the precision on $\lambda$ is increased with new UCN measurements, corresponding reductions in the error on $\tau_{n}$ will become important. A comprehensive determination of the complete set of neutron decay correlation coefficients using a beam of pulsed cold neutrons at the SNS has also been considered. Such an experiment would allow a redundant determination of $\lambda$, with multiple cross checks on systematic effects, thereby providing additional confidence in the value of this quantity. For a recent theoretical study of neutron decay correlation coefficients, see Ref. [224]. For other theoretical considerations, see Ref. [225].

To date, the most precise determination of $\left|V_{u d}\right|$ has been obtained from analysis of "super-allowed" Fermi nuclear $\beta$ decays. These decays involve transitions from spin-parity $J^{\pi}=0^{+}$initial states to $0^{+}$ daughter nuclei and, thus, only matrix elements of the vector part of the charge changing weak current may contribute. In the limit of zero momentum transfer squared, the latter is simply the isospin raising or lowering operator, and to the extent that the states involved in the transition are part of an isospin 
multiplet, the matrix element $\left\langle M_{V}\right\rangle$ of the time component of the charge changing vector current is

$$
\left\langle I, I_{Z} \pm 1\left|J_{0}^{ \pm}\right| I, I_{Z} 1\right\rangle=\left[\left(I \mp I_{Z}\right)\left(I \pm I_{Z}+1\right)\right]^{1 / 2}
$$

independently of nuclear structure. Small deviations from this exact isospin symmetric result are induced by configuration mixing as well as small differences in the neutron and proton radial wavefunctions in the initial and final nuclear states. The corresponding corrections, along with those due to nuclear structure dependent effects in EW radiative corrections, must be computed and applied to the $f t$ values before a nucleus independent value of $G_{F}^{\beta}$ is obtained. The resulting expression for the corrected $f t$ values, $\mathcal{F} t$, is given by,

$$
\mathcal{F} t\left(1+\delta_{R}\right)\left(1-\delta_{C}\right)=\frac{K}{2\left(G_{F}^{\beta}\right)^{2}},
$$

where $\delta_{R}$ is a nucleus dependent component of the $\mathcal{O}(\alpha)$ EW radiative corrections, and $\delta_{C}$ is a nuclear structure correction that enters because the initial and final nuclear states are not perfect isospin multiplet partners. The latter correction accounts for two effects, configuration mixing in the nuclear states and small differences in the initial and final radial wave-functions.

An important test of the nuclear structure calculations required for $\delta_{R}$ and $\delta_{C}$ is a comparison of the $\mathcal{F} t$ values from different decays, which should agree according to the approximate conserved vector current $(\mathrm{CVC})$ property of the SM. The $\mathcal{F} t$ values for nine different super-allowed transitions have been measured with a precision of $0.1 \%$ or better (for recent reviews, see Refs. 226 228]). A fit [227] yields $\mathcal{F} t=3072.2 \pm 0.8$, indicating agreement with $\mathrm{CVC}$ at the level of a few parts in $10^{4}$. The largest contributors to the overall uncertainty are the estimated uncertainties in $\delta_{R}$ and $\delta_{C}$.

In order to extract a value of $\left|V_{u d}\right|$ from the average $\mathcal{F} t$ values, the calculated correction, $\Delta r_{\beta}-\Delta r_{\mu}$, must also be subtracted, as indicated in Eq. (88). In particular, the correction $\Delta r_{\beta}$ is sensitive to non-perturbative QCD effects, introducing an additional source of uncertainty beyond those appearing in the average $\mathcal{F} t$ value. This uncertainty arises primarily from the box diagram of Figure 4 involving the exchange of one $W^{ \pm}$boson and one $\gamma$ that yields the amplitude,

$$
\mathcal{M}_{W \gamma}=\frac{G_{\mu}}{\sqrt{2}} \frac{\hat{\alpha}}{2 \pi}\left[\ln \left(\frac{M_{W}^{2}}{\Lambda^{2}}\right)+C_{\gamma W}(\Lambda)\right],
$$

where the leading logarithmic term can be computed reliably in the SM and the constant $C_{\gamma W}(\Lambda)$ parameterizes contributions to the loop integral below a scale $\Lambda$. An estimate of $C_{\gamma W}(\Lambda)$ was given by Sirlin 211] using nucleon intermediate states in the box diagram, and this estimate has been retained by subsequent authors. To estimate the corresponding uncertainty, Ref. [226] used the response of the logarithmic term in Eq. (95) when $\Lambda$ is varied between 400 and $1600 \mathrm{MeV}$. One obtains 228],

$$
\left|V_{u d}\right|=0.9740 \pm 0.0005
$$

where the error is dominated by the estimated uncertainty in $\Delta r_{\beta}( \pm 0.0004)$. When the results from neutron decay are averaged with the result (96), one obtains the PDG [113] value ${ }^{12}$,

$$
\left|V_{u d}\right|=0.9734 \pm 0.0008 .
$$

Obtaining a more refined, first principles computation of $C_{\gamma W}(\Lambda)$ and the attendant theoretical uncertainty remains an open theoretical problem.

One aim of on-going work in the arena of super-allowed decays is to provide further tests of the nuclear corrections $\delta_{R}$ and $\delta_{C}$. To that end, a comprehensive calculation of these corrections for medium-

\footnotetext{
${ }^{12}$ The Particle Data Group has augmented the uncertainty in (96) by a factor of two to account for a possible $Z$ dependence of the super-allowed $\mathcal{F} t$ values, although there exists no statistically significant evidence for such an effect.
} 
and heavy-mass nuclei has been carried out [229]. The computations include both the measured cases as well as new transitions whose measurement could provide a test of these calculations. A program to determine the $f t$ values for a number of these new cases with $18 \leq A \leq 42$ is underway at Texas A \& M University and TRIUMF [228. Studies of heavier nuclei, such as ${ }^{74} \mathrm{Rb}$ or ${ }^{62} \mathrm{Gd}$ present new experimental and theoretical challenges and may prove problematic for testing the reliability of $\delta_{R}$ and $\delta_{C}$ computations for the known cases. These challenges include the rapid shape changes of nuclei in the $A \geq 62$ region, the short half-lives of the parent nuclei, and the plethora of $1^{+}$daughter states whose branching fractions must be measured accurately in order to extract $\mathcal{B}\left(0^{+} \rightarrow 0^{+}\right)$[228].

To provide a test of the unitarity requirement in Eq. (85), one must have in hand reliable values for $\left|V_{u s}\right|$ and $\left|V_{u b}\right|$. The value of $\left|V_{u b}\right|=0.0032 \pm 0.0009$ is sufficiently small that it may be neglected for this purpose. In contrast, the uncertainty in the currently accepted value of $\left|V_{u s}\right|$ [113],

$$
\left|V_{u s}\right|=0.2196 \pm 0.0026
$$

extracted from kaon leptonic decays $\left(K_{e 3}\right)$ has a similar impact on the unitarity test as does the uncertainty in $\left|V_{u d}\right|$. Using the values (9798) one obtains for the first row of the CKM matrix,

$$
\sum_{j=d, s, b}\left|V_{u j}\right|^{2}=0.9958 \pm 0.0019
$$

indicating a $2.2 \sigma$ deviation from unitarity.

Recent experimental and theoretical analyses of $\left|V_{u s}\right|$ have raised questions about both the central value and quoted error in this quantity. A new analysis of $\sim 70,000$ charged kaon decay events has been carried out by the Brookhaven E865 Collaboration [230, leading to $\left|V_{u s}\right|=0.2272 \pm 0.0023 \pm 0.0019$. The first error is the combined statistical and systematic error in the partial width $d \Gamma\left(K_{e 3}^{+}\right)$while the second error is associated with the transition form factor, $f_{+}(t)$, where $t=\left(p_{K}-p_{\pi}\right)^{2}$. Both the value of $f_{+}(0)$ and its slope at the photon point, $t=0$, are needed to extract $\left|V_{u s}\right|$ from the partial width,

$$
d \Gamma\left(K_{e 3}^{+}\right)=C(t)\left|V_{u s}\right|^{2}\left|f_{+}(0)\right|^{2}\left[1+\lambda_{+} \frac{t}{m_{\pi}^{2}}\right]^{2},
$$

where $C(t)$ is a known function and where QED radiative corrections (not shown here) must also be included in order to determine $\left|V_{u s}\right|$ to the level of precision needed 231. As can be seen, the slope of $f_{+}(t)$ at the photon point is characterized by $\lambda_{+}$.

Independent experimental determinations of $\lambda_{+}$have been obtained from studies of $K_{\ell 3}$ decays [113]. The value of $f_{+}(0)$ requires theoretical input. A determination to $\mathcal{O}\left(p^{4}\right)$ in $\chi \mathrm{PT}$ was carried out in Refs. 232 233], while an estimate of the $\mathcal{O}\left(p^{6}\right)$ terms was obtained using the quark model. The uncertainty associated with the latter was estimated to be roughly $1 \%$. More recent computations have explicitly evaluated the $\mathcal{O}\left(p^{6}\right)$ contributions [234235]. While the one- and two-loop contributions can be evaluated using the known low energy constants (LECs) through $\mathcal{O}\left(p^{4}\right)$, the contributions from the $\mathcal{O}\left(p^{6}\right)$ LECs cannot be determined with sufficient precision from existing data. In particular, the $\mathcal{O}\left(p^{6}\right)$ contribution to $f_{+}(0)$ contains [235],

$$
f_{+}^{(6)}=-8 \frac{\left(m_{\pi}^{2}-m_{K}^{2}\right)^{2}}{F_{\pi}^{4}}\left(C_{12}^{r}+C_{34}^{r}\right)+\cdots,
$$

where the $C_{i}^{r}, i=12,34$, are two of the $94 \mathcal{O}\left(p^{6}\right)$ LECs.

Naive dimensional arguments, as well as those invoking resonance saturation of relevant pseudoscalar form factors suggest that the impact of the $C_{i}^{r}$ on the extracted value of $\left|V_{u s}\right|$ could be substantially larger than the errors quoted for either the PDG value or the recent Brookhaven result. New measurements of pion and kaon form factors, however, could reduce the uncertainty in $\left|V_{u s}\right|$ from the $C_{i}^{r}$ to below one 
percent. As demonstrated in Ref. [235], a precise measurement of the pion scalar form factor would allow a sufficiently precise determination of $C_{12}^{r}$, while new determinations of $\lambda_{0}$, the slope parameter in the kaon scalar form factor, using $K_{\mu 3}$ decays, would provide a value for $2 C_{12}^{r}+C_{34}^{r}$. Together, these experimental inputs would yield the combination, $C_{12}^{r}+C_{34}^{r}$, needed for $f_{+}(0)$.

Experimental work in this direction is underway at several facilities. Of particular interest are kaon decay branching ratio measurements being performed by the KLOE experiment [236] at DA $\Phi$ NE. Preliminary results for the branching ratios of the $K_{S, L} \rightarrow \pi^{-} e^{+} \nu_{e}$ and $K_{L} \rightarrow \pi^{-} \mu^{+} \nu_{\mu}$ channels have been reported in Ref. [236]. To the extent that isospin is a good symmetry, the form factors $f_{+}^{K^{0} \pi^{-}}(t)$ and $f_{+}^{K^{+} \pi^{0}}(t)$ should be identical at $t=0$. The isospin corrections have been computed in $\chi \mathrm{PT}$ to $\mathcal{O}\left(p^{4}\right)$ and found to be $\sim 2 \%$ [233. A comparison of the product $\left|V_{u s}\right| f_{+}^{K^{0} \pi^{-}}(0)$ for the preliminary KLOE results of the three different neutral kaon branching ratios with the previously obtained PDG world averages indicates good agreement. The results differ substantially, however, with the E865 result for $\left|V_{u s}\right| f_{+}^{K^{+} \pi^{0}}(0)$, which also differs from the previous world average for this quantity. The situation may be clarified by future KLOE results for the $K_{e 3}^{+}$branching ratio as well as studies planned by the NA48 [237] and KTeV 238] Collaborations. Given the present disagreement, as well as the theoretical uncertainties associated with the $\mathcal{O}\left(p^{6}\right)$ LECs, it is probably too soon to conclude that the disagreement with CKM unitarity has been resolved by a new value of $\left|V_{u s}\right|$.

Should the present unitarity disagreement persist, then one could draw interesting conclusions about NP. In the context of gauge unification, a small degree of mixing between left- and right-handed gauge bosons could restore the required unitarity. Because $G_{F}^{\beta}$ from which $V_{u d}$ is derived parameterizes a vector coupling of the $W^{ \pm}$to quarks, it is insensitive to the degree to which the boson is a mixture of $W_{L}^{ \pm}$and $W_{R}^{ \pm}$. In contrast, $G_{F}^{\beta}$ does depend on the chirality of the outgoing leptons. Because the effect of a right-handed component to the neutrino is highly suppressed, the coupling of the lightest $W^{ \pm}$mass eigenstate to the left-handed leptons will differ from the $W_{L}^{ \pm}$coupling by an amount proportional to the left-right mixing angle, $\xi$. Thus, the present unitarity disagreement would imply a non-zero value for $\xi$ at the $95 \%$ CL. Such a result would have implications for the interpretation of other precision measurements, such as neutrinoless double $\beta$ decay (see Section [5.6).

First row CKM unitarity has also important implications for SUSY. The results of a recent analysis of MSSM radiative corrections to various CC observables [186] indicate that inclusion of these corrections typically exacerbates the disagreement with the unitarity requirement when the SUSY breaking parameters are chosen in accord with the most common models of SUSY breaking mediation. The region of parameter space favored by weak decays, $(g-2)_{\mu}$, the current values of $M_{W}, m_{t}$, and the lightest Higgs mass along with theoretical considerations such as color neutrality of the vacuum implies that the mass of the left-handed muon superpartner is heavier than that of the first generation scalar quarks, in contrast to expectations based on gravity- and gauge-mediated SUSY breaking. Precision CC data can accommodate SUSY radiative corrections under the assumptions of these models if the requirement of $R_{P}$ conservation is relaxed 98170239. This would not be attractive from the standpoint of cosmology, since the RPV interactions that contribute to weak decays would also mediate the decay of the $\chi^{0}$, ruling it out as a candidate for cold dark matter. A test of this scenario could be performed through a comparison of PV electron scattering experiments [239240].

\section{Rare and Forbidden Processes}

\subsection{Electric Dipole Moments}

Processes that violate $\mathrm{CP}$ and $\mathrm{T}$ have been an important arena of study for SM physics. Within the $\mathrm{SM}$, the CP violation observed in the decays of neutral kaons is accommodated by the presence of the CP violating phase in the CKM matrix. Recently, experimental results from the NA48 [241] and 
$\mathrm{KTeV}$ [242] Collaborations have confirmed the SM prediction for the ratio of $\epsilon^{\prime} / \epsilon$ that expresses the relative strength of direct $\mathrm{CP}$ violation in the decay amplitudes compared to that associated with $K^{0}$ $\bar{K}^{0}$ mixing. With a few possible expceptions under study, CP violation in the $b$ quark system is also in agreement with the SM. Thus, there appears to be little evidence at present for new sources of CP violation in these channels.

Searches for $\mathrm{CP}$ and $\mathrm{T}$ violation in light quark and lepton systems have, to date, produced null results. The most powerful probes of this type are searches for the permanent electric dipole moments (EDMs) of leptons, neutrons, and neutral atoms. EDM searches are of interest for several reasons:

- The SM (CKM) predictions for the magnitudes of EDMs are suppressed, falling well below the sensitivity of present and prospective measurements. Consequently, the observation of a non-zero EDM could signal the presence of physics beyond the SM or CP violation in the $S U(3)_{C}$ sector of the SM. The latter arises via a term in the Lagrangian [4,

$$
\mathcal{L}_{\text {strong } \mathrm{CP}}=\theta_{\mathrm{QCD}} \frac{\alpha_{s}}{8 \pi} G_{\mu \nu} \tilde{G}^{\mu \nu},
$$

where $G_{\mu \nu}\left(\tilde{G}_{\mu \nu}\right)$ is the (dual) $S U(3)_{C}$ field strength tensor.

- The observed predominance of matter over anti-matter in the universe - the so-called baryon asymmetry of the universe (BAU) - conflicts with expectations based on the SM alone. In particular, the strength of $\mathrm{CP}$ violating effects needed to preserve the matter-antimatter asymmetry during the evolution of the universe is suppressed in the SM by the Jarlskog invariant [243],

$$
J=\cos \theta_{1} \cos \theta_{2} \cos \theta_{3} \sin \theta_{1}^{2} \sin \theta_{2} \sin \theta_{3} \sin \delta,
$$

with the $\theta_{i}$ and $\delta$ being the angles in the CKM matrix, and by light quark masses, rendering a BAU that is far smaller than observed ${ }^{13}$. On the other hand, candidate extensions of the SM that could provide new $\mathrm{CP}$ violation of sufficient strength to accommodate the BAU could also generate EDMs large enough to be seen experimentally.

The literature on EDMs is vast, so we make no attempt to provide an exhaustive review here (for recent reviews, see Refs. [244-247]). Instead, we highlight the most important experimental developments and theoretical issues for the field, and point the reader to other studies for more comprehensive reviews. From our standpoint, three aspects of the EDM program merit emphasis:

- Recent experimental developments have put the field on the verge of a revolution. Experimental searches for the electron, muon, neutron, and atomic EDMs are poised to improve experimental sensitivity by factors of 100 to 10,000 during the next decade. This kind of across-the-board improvement in precision by orders of magnitude has never before been seen in the field.

- The lepton, neutron, and atomic EDM searches provide complementary probes of new CP violation, as different candidate theories imply different signatures for the various moments. For example, the observation of a non-zero neutron or atomic EDM in conjunction with a null result for the electron EDM at a comparable level of sensitivity would point toward the interaction of Eq. (102) as the likely source of CP violation. In contrast, a non-zero lepton EDM would be a smoking gun for $\mathrm{CP}$ violation outside the SM, and a comparison with neutron and atomic studies would be essential for identifying the particular scenario responsible.

\footnotetext{
${ }^{13}$ The SM also does not produce a sufficiently strong first order phase transition needed for the BAU.
} 
Table 9: Present and prospective EDM limits. Expectations based on SM (CKM) CP violation are also shown.

\begin{tabular}{|c|c|c|c|c|}
\hline System & Present Limit $(e-\mathrm{cm})$ & Group & Future Sensitivity & Standard Model (CKM) \\
\hline $\begin{array}{l}e^{-} \\
e^{-} \\
e^{-}\end{array}$ & $1.6 \times 10^{-27}(90 \% \mathrm{CL})$ & $\begin{array}{l}\text { Berkeley } \\
\text { Yale } \\
\text { LANL }\end{array}$ & $\begin{array}{l}\sim 10^{-29} \\
\sim 10^{-30}\end{array}$ & $<10^{-38}$ \\
\hline $\begin{array}{l}\mu \\
\mu\end{array}$ & $1.05 \times 10^{-18}(90 \% \mathrm{CL})$ & $\begin{array}{l}\text { CERN } \\
\text { BNL }\end{array}$ & $\sim 10^{-24}$ & $<10^{-36}$ \\
\hline $\begin{array}{l}n \\
n \\
n\end{array}$ & $6.3 \times 10^{-26}(90 \% \mathrm{CL})$ & $\begin{array}{l}\text { ILL } \\
\text { PSI } \\
\text { LANL }\end{array}$ & $\begin{array}{r}1.5 \times 10^{-26} \\
7 \times 10^{-28} \\
2 \times 10^{-28}\end{array}$ & $1.4 \times 10^{-33} \rightarrow 1.6 \times 10^{-31}$ \\
\hline $\begin{array}{c}{ }^{199} \mathrm{Hg} \\
{ }^{225} \mathrm{Ra} \\
{ }^{129} \mathrm{Xe} \\
D\end{array}$ & $2.1 \times 10^{-27}(95 \% \mathrm{CL})$ & $\begin{array}{l}\text { Seattle } \\
\text { Argonne } \\
\text { Princeton } \\
\text { BNL }\end{array}$ & $\begin{array}{r}5 \times 10^{-28} \\
10^{-28} \\
10^{-31} \\
\sim 10^{-27}\end{array}$ & $\begin{array}{l}\lesssim 10^{-33} \\
\lesssim 10^{-34}\end{array}$ \\
\hline
\end{tabular}

- The precise implications of EDM measurements for the $\mathrm{CP}$ violation needed for the BAU remains an open theoretical problem. If, for example, CP violation arises in the lepton sector via mixing of Majorana neutrinos (see Section [5.1), then it could produce a BAU via $B-L$ conserving interactions that both transform $L$ violation into $B$ violation and transmit the $\mathrm{CP}$ violating effects into the baryon sector. Presumably, such processes occur at high scales associated with the see-saw mechanism 248 250] (see Section 5.1) making them difficult to translate into precise, weak scale computations as required for the study of EDMs. Even the more conventional EWB remains subject to unquantified approximations and theoretical uncertainties, rendering the relationship between the BAU and EDMs somewhat opaque.

In what follows, we review recent developments relevant to each of these points.

\subsubsection{Experimental developments}

Each EDM search relies on the same experimental signature of a non-zero EDM, namely, a small shift in the Larmour precession frequency in the presence of an applied electric field,

$$
\hbar \omega=-\mu \vec{J} \cdot \vec{B}-d \vec{J} \cdot \vec{E}
$$

where $\mu$ and $d$ are the magnetic and electric dipole moments of the system of interest, $\vec{J}$ is its spin, and $\vec{B}$ and $\vec{E}$ are the applied magnetic and electric fields. Under reversal of the direction of $\vec{E}$, the contribution from the EDM to the precession reverses sign, thereby allowing one to isolate the tiny EDM induced shift from the much larger effect of the magnetic moment. The challenge for experimenters is to apply electric fields with as large a magnitude as possible, thereby enhancing the sensitivity to $d$, while minimizing various systematic effects, such as leakage currents, that can mimic the effect of the $d \vec{J} \cdot \vec{E}$ interaction. In many cases, the quest for improved sensitivity is aided by various fortuitous enhancement factors that can amplify one's EDM sensitivity. The present experimental limits on the EDMs of various particles are listed in Table 9. 


\section{Electron}

The most precise limit on the electron EDM, $d_{e}$, has been achieved by the Berkeley group with a measurement of atomic Thallium [251]. The extraction of this limit from the atomic EDM relies on an observation by Sandars 252 that the EDM of a paramagnetic atom, $d_{A}$, induced by an electron EDM can be substantially enhanced. For Tl, the enhancement factor is $R=d_{e} / d_{A}=-585$ [253]. The experiment employed a pair of atomic beams traversing identical paths but experiencing applied electric fields with the same magnitude and opposite sign. One beam consisted of sodium atoms that served as a co-magnetometer used to identify and minimize systematic effects. Interchanging the paths traversed by the $\mathrm{Na}$ and $\mathrm{Tl}$ beams would lead to a phase difference $\delta_{\mathrm{EDM}}$ for the two Tl paths due to a non-vanishing $d_{e}$, but no phase difference for the spin-0 Na beam. The absence of any phase difference leads to a limit on $\left|d_{e}\right|$. Corrections were applied for residual, motional magnetic field and geometric phase effects, and conservative upper bounds placed on leakage current, charging current, and dielectric absorption. An applied field of strength 410 stat-volts/cm was used. From the calculated paramagnetic enhancement factor $R$ and the upper limit on the phase $\delta_{\mathrm{EDM}}$ the Berkeley group obtained the $90 \% \mathrm{CL}$ limit given in Table 9 .

Further improvements in $\left|d_{e}\right|$ sensitivity are being pursued by two groups. The Yale group is employing a $\mathrm{PbO}$ molecule, for which near degeneracies of opposite parity molecular states enhance the electric field experienced by molecular electrons by two or more orders of magnitude relative to the corresponding enhancement in atoms [254255]. The possibility for such enhancements in polar molecules was first observed by Sandars [256] and subsequently applied to molecules such as PbO [257 260]. The Yale experiment will rely on the $a(1)$ states in $\mathrm{PbO}$, where a degeneracy between states with electronic angular momentum projection along the internuclear axis, $\vec{J}_{e} \cdot \hat{n}= \pm 1$ is lifted by a Coriolis coupling between $J_{e}$ and molecular rotational angular momentum. The splitting for this state is $\Delta \Omega_{J}=11.2(2) \mathrm{MHz}$. The presence of a non-zero $d_{e}$ in an applied electric field will lead to a shift in the $M= \pm 1$ projections of the two $a(1)$ states. The Yale group has recently demonstrated the feasibility of this technique by studying Zeeman induced shifts in the $M$ sub-levels and by observing the Stark shift in the $J=1$ doublet in the presence of a small $(|\vec{E}|<2 \mathrm{~V} / \mathrm{cm})$ applied field [255]. For a field of this magnitude, the $\mathrm{PbO}$ measurement would be sensitive to an electron EDM at the scale of the current experimental limit. The group anticipates that implementation of various experimental improvements will lead to an EDM sensitivity of $\sim 10^{-29} e$-cm in a month of running.

The Los Alamos group is pursing a $d_{e}$ measurement using a solid state technique [261]. The basic idea, originally developed by Shapiro [262], relies on changes in the magnetic flux $\Phi_{\mu}$ in a macroscopic

solid state sample when the orientation of an external $\vec{E}$ is reversed. If the electrons in the solid have a nonzero EDM, then the application of $\vec{E}$ will cause the electron spins to align, thereby inducing a magnetic flux. Thus, by searching for changes in $\Phi_{\mu}$ when the sign of $\vec{E}$ is reversed, probes for a nonzero $d_{e}$. The Collaboration hopes to achieve a sensitivity of $10^{-30} e-\mathrm{cm}$ in ten days of integration using Gadolinium Gallium Garnet polycrystalline material.

\section{Muon}

Although the present limits on $d_{\mu}$ fall well below SM expectations, a measurement at the level of $10^{-24} e$-cm may be feasible in a storage ring experiment [263. Such an experiment would rely on a $g-2$ precession type set-up, but with an applied, radial electric field that would cancel the $g-2$ in plane precession. The precession frequency is given by,

$$
\vec{\omega}=\frac{3}{m_{\mu}}\left[a \vec{B}+\left(\frac{1}{\gamma^{2}-1}-a\right) \frac{\vec{\beta} \times \vec{E}}{c}+\frac{\eta}{2}(\vec{E} c+\vec{\beta} \times \vec{B})\right]
$$


where $a$ is the muon anomalous magnetic moment and $\eta$ is related to the muon EDM via,

$$
d_{\mu}=\frac{\eta}{2} \frac{e \hbar}{2 m_{\mu} c} \approx 4.7 \times 10^{-11} e-\mathrm{cm}
$$

For the $g-2$ measurements, the muon energy is tuned to give the "magic" $\gamma=29.3$ that eliminates the second term in Eq. (105), leaving the precision entirely to $a$. The EDM measurement would use lower energy muons and choose the value of the radial electric field $\vec{E}$ so as to cancel the first two terms. The resulting precession would arise entirely from the third term and would cause the spin to tilt out of plane. The degree of this tilt would be measured by exploiting the parity violation in the muon decay and looking for a vertical asymmetry in the number of decay positrons.

\section{Neutron}

The first searches for the permanent EDM of a quantum system were carried out with neutrons in the pioneering work of Purcell and Ramsey [264265. The sensitivity of the original experiment has been steadily improved upon, culminating in the present limit of $6.3 \times 10^{-26} e$-cm achieved at ILL using ultra-cold neutrons (UCNs) [266]. The ILL Collaboration achieved a density of $0.6 \mathrm{UCN} / \mathrm{cm}^{3}$. The technique involved observing the shift in the Larmour precession when the direction of the applied electric field was reversed relative to the static magnetic field. A Hg co-magnetometer was used to keep track of magnetic field fluctuations. The Collaboration expects to improve this sensitivity by roughly a factor of four by increasing the number of UCNs collected and stored [267].

Future, even more sensitive searches will be carried out by several groups. An experiment based at LANSCE [268] will use a technique involving super cooling the neutrons by down-scattering in ${ }^{4} \mathrm{He}$ and observing the neutron spin precession relative to that of a dilute mixture of ${ }^{3} \mathrm{He}$ that will also be present in the cells. The relative alignment of the neutron and ${ }^{3} \mathrm{He}$ spins will be measured by observing the spin dependent neutron capture on ${ }^{3} \mathrm{He}$. The ${ }^{3} \mathrm{He}$ will also serve as co-magnetometers. The Collaboration hopes to achieve a density of $500 \mathrm{UCN} / \mathrm{cm}^{3}$. The initial goal of the experiment is a sensitivity of $9 \times 10^{-28} e-\mathrm{cm}$ at LANL with a final target of better than $2 \times 10^{-28} e-\mathrm{cm}$ to be reached at the SNS.

An experiment at PSI is being developed that will use a solid $\mathrm{D}_{2}$ source to produce a density of $1000 \mathrm{UCN} / \mathrm{cm}^{3}$ [269]. The technique involves employing two adjacent UCN cells in which the applied electric fields have opposite orientation. Neighboring cells of cesium will serve as co-magnetometers. The goal of the experiment is a sensitivity of $5 \times 10^{-28} e-\mathrm{cm}$.

\section{Neutral Atoms}

The most stringent limit on the permanent EDM of any quantum system has been achieved for the

${ }^{199} \mathrm{Hg}$ atom by the Seattle group, $\left|d_{A}\right|<2.1 \times 10^{-27} e-\mathrm{cm}[270$. The experiment relied on observing the Zeeman precession frequency of the ${ }^{199} \mathrm{Hg}$ nuclear spin $\vec{I}$ in the presence of magnetic and electric fields. Two adjacent ${ }^{199} \mathrm{Hg}$ cells were used. The orientation of the magnetic fields in the two cells was the same, but that of the electric field was opposite. A comparison of the precession frequency in the two cells provided a probe for the $d_{A} \vec{I} \cdot \vec{E}$ interaction. An improved version of this experiment is underway that uses as magnetometers two additional cells that do not experience the applied $\vec{E}$. A $100 \% \mathrm{CO}$ buffer gas will increase the $\mathrm{Hg}$ spin coherence time [160]. The Collaboration anticipates a factor of four improvement over the current limit.

An alternate strategy being employed at Princeton will use liquid ${ }^{129} \mathrm{Xe}$ [271]. The technique will rely on long range dipole interactions that amplify magnetic field gradients. Such gradients would be induced by an applied $\vec{E}$ that, in the presence of a non-zero atomic EDM, would lead to spatial gradients in the associated shift in the precession frequency. Such frequency gradients, in turn, would induce magnetic field gradients that get amplified in the ${ }^{129} \mathrm{Xe}$ liquid, which serves as its own magnetometer. The 
Collaboration hopes to probe the ${ }^{129} \mathrm{Xe}$ EDM with several orders of magnitude better precision than obtained in the ${ }^{199} \mathrm{Hg}$ experiment [272].

A group at Argonne is pursuing a measurement of the ${ }^{225} \mathrm{Ra}$ EDM using optical trapping [273]. As discussed in the next subsection one expects the EDM of ${ }^{225} \mathrm{Ra}$ to be $\sim 400$ times larger than that of ${ }^{199} \mathrm{Hg}$ for a given source of CP violation in the nucleus. The optical trapping technique allows one both to reduce systematic effects as well as apply large external electric fields. The Argonne group hopes to achieve an EDM with sensitivity of $\sim 10^{-28} e$-cm. Similar ${ }^{225}$ Ra searches are also being pursued at KVI and TRIUMF. For a brief review of these efforts, see Ref. [274]

\section{Deuteron}

Recently, the possibility of measuring the deuteron EDM using a storage ring experiment similar to that for the muon EDM experiment has received considerable attention [263]. Measuring $d_{D}$ presents several experimental challenges not present in the case of the muon: the deuteron does not decay, so the out of plane rotation of its spin must be detected via the scattering from protons or carbon; its anomalous magnetic moment is two orders of magnitude larger than that of the muon; its spin coherence time is of the order of $10 \mathrm{~s}$; and the deuteron carries spin one, so that it can have both vector and tensor polarizations. Efforts are underway to address these challenges, but initial estimates suggest that a measurement with $\sim 10^{-27} e$-cm may be feasible at existing or future storage rings [263].

\subsubsection{Theoretical implications}

The SM contains two sources of CP violation that can generate an EDM, the phase $\delta$ in the CKM matrix and the gluonic operator in Eq. (102). The magnitude of theoretical EDM predictions derived from CKM $\mathrm{CP}$ violation - listed in Table 9 - fall well below the present and prospective experimental bounds. The CKM induced EDMs of leptons and quarks vanish to two-loop order and are thus suppressed [275277. Naive dimensional analysis (NDA) leads to an estimate for the electron EDM,

$$
d_{e} \sim e \frac{\alpha_{s}\left(M_{W}\right) G_{F}^{2} M_{W}^{2} m_{e}}{\pi^{5}} J \frac{m_{c}}{m_{t}} \frac{m_{s}}{m_{b}} \sim \times 10^{-35} e-\mathrm{cm},
$$

where the presence of $\alpha_{s}$ is due to gluonic corrections that appear at three-loop order. Scaling the NDA estimate in Eq. (107) by $m_{\mu} / m_{e}$ gives a conservative estimate for $d_{\mu}$ of $\sim 10^{-33} e$-cm. Smaller values, also obtained from dimensional arguments, also appear in the literature [277] and are used in Table 9 .

The corresponding predictions for the quark EDMs range from $10^{-31}$ to $10^{-33} e-\mathrm{cm}[276]$. The valence quark contribution to the neutron EDM is given by,

$$
d_{n}=\frac{1}{3}\left(4 d_{d}-d_{u}\right)
$$

so that one might expect the SM (CKM) contribution to the neutron EDM to be of the same order of magnitude. Chiral corrections to Eq. (108) may lead to order of magnitude enhancements of $d_{n}$. These effects arise via pseudoscalar loops containing $\Delta S=1$ weak interactions at the meson-baryon vertices that are singular in the chiral limit [278 280]. A calculation of such contributions contains significant uncertainties, however, due to present limitations on carrying out first principles computations of the weak baryon-pseudoscalar meson vertices. Typically, they rely on approximations of questionable validity, such as factorization and vacuum saturation. Moreover, the CKM induced EDM — which is second order in the $\Delta S=1$ weak interaction - requires inclusion of intermediate states containing strange hadrons. The convergence of the $S U(3)$ chiral expansion for baryons is slow at best, and higher order operators omitted from the computations of Refs. 278 280] may make significant contributions. Nevertheless, these studies provide a rough guide to the possible magnitude of $d_{n}$, so for illustrative purposes, we quote the range from Ref. 280] in Table 9 
Within the context of CKM CP violation, the EDMs of nuclei and atoms such as ${ }^{199} \mathrm{Hg}$ are generated primarily by $\mathrm{T}$ - and P-odd $N N$ interactions, $\hat{H}_{N N}^{P, T}$, in the nucleus. Early work in this regard has been carried out in Refs. [281282, where it was noted that a long range, P- and T-odd nuclear force due to CKM CP violation could appear in the guise of kaon exchange between the two nucleons. The presence of kaons is necessary because the CKM CP violating interaction in light quark systems must be second order in the $\Delta S=1$ weak interaction. In this case, the $\mathrm{P}$ - and T-odd nuclear force takes on the form,

$$
\hat{H}_{N N}^{P, T}=\frac{G_{F}}{\sqrt{2}} \frac{1}{2 m_{N}} \sum_{a b}\left[\left(\eta_{a b} \vec{\sigma}_{a}-\eta_{b a} \vec{\sigma}_{b}\right) \cdot \vec{\nabla}_{a} \delta\left(\vec{r}_{a}-\vec{r}_{b}\right)+\eta_{a b}^{\prime}\left(\vec{\sigma}_{a} \times \vec{\sigma}_{b}\right) \cdot\left\{\left(\vec{p}_{a}-\vec{p}_{b}\right), \delta\left(\vec{r}_{a}-\vec{r}_{b}\right)\right\}\right],
$$

where the sum is over the combinations, $p p, n n$, and $n p$. Ref. [282] estimated the values of the $\eta_{a b}$ to be of order $10^{-8}$. However, this estimate did not properly take into account the structure of chiral symmetry and likely represents an overestimate of an order of magnitude or more [283].

A complication arises in relating $\hat{H}_{N N}^{P, T}$ to an atomic EDM. As first noted by Schiff [284], the effect of an external electric field $\vec{E}$ on a point-like nucleus with an EDM would be entirely screened out by the field created by distortions induced by $\vec{E}$ in the atomic electron cloud. Classically, this effect can be understood by noting that there can be no net acceleration of a neutral atom in the presence of $\vec{E}$, so the acceleration that would be caused by the effect of $\vec{E}$ on the charged nucleus must be canceled by a corresponding field created by re-arrangements of the atomic electrons. Corrections to this "Schiff screening" arise from several sources, including finite nuclear size, modifications of the magnetic electron-nucleus interaction due to $\vec{E}$ induced atomic orbital distortion, and higher P- and T-odd nuclear moments, such as the magnetic quadrupole moment. In the case of ${ }^{199} \mathrm{Hg}$, only the first two effects are relevant since the nuclear spin is $I=1 / 2$. To date, most authors have concentrated on the effects of finite nuclear size. The corresponding effect on the atomic EDM is driven by the so-called Schiff moment, which may be thought of as an $r^{2}$-weighted moment of a P-and T-odd component of the nuclear charge density. Ref. [282 has provided a simple estimate of the ${ }^{199} \mathrm{Hg}$ EDM and Schiff moment $(Q)$, using a simplified, schematic model for the nuclear wave-function,

$$
d_{A}\left({ }^{199} \mathrm{Hg}\right)=-4 \times 10^{-17}\left(\frac{Q}{e \mathrm{fm}^{3}}\right) e-\mathrm{cm}, \quad Q\left({ }^{199} \mathrm{Hg}\right)=-1.4 \times 10^{-8} \eta_{a b} \text { e } \mathrm{fm}^{3} .
$$

Using the revised estimate, $\eta_{a b} \sim 10^{-9}$, a CKM induced EDM for mercury of a few $\times 10^{-34} e$-cm is expected, which is well below the current and prospective sensitivities.

Although the effect of CKM CP violation is unlikely to ever be observed in ${ }^{199} \mathrm{Hg}$, other atoms may offer the possibility of enhanced EDM effects. As emphasized in Ref. [281], the effect of $\hat{H}_{N N}^{P, T}$ in certain nuclei can be amplified by accidents in nuclear structure. The effect arises because the P- and T-odd nuclear force mixes states of opposite parity, and for certain nuclei the ground state is part of a nearlydegenerate parity doublet. The strength of wrong parity admixture into the ground state is enhanced by the small energy splitting. Ref. 281 computed the corresponding enhancement factors in the context of the gluonic CP violation of Eq. (102), but did not consider ${ }^{199} \mathrm{Hg}$. Moreover, the authors focused exclusively on the enhancements of the nuclear EDMs, which are not directly observable in atomic EDM experiments, rather than on the Schiff moments that are relevant in these cases. Nonetheless, one naively expects enhancements of the Schiff moments in cases where the nuclear EDMs are also amplified. For non-spherical nuclei the effect can be particularly pronounced, leading to enhancements of two to three orders of magnitude.

Recently, the nuclear enhancements of the Schiff moment was analyzed for ${ }^{225} \mathrm{Rn}$ [285] (see also Refs. [286 287]), an octupole deformed nucleus whose atomic EDM measurement is being pursued by the Argonne group. The analysis [285] reveals an enhancement of $Q\left({ }^{225} \mathrm{Rn}\right)$ relative to $Q\left({ }^{199} \mathrm{Hg}\right)$ by a factor of several hundred, using the work of Ref. 288 on the latter for comparison. While such an enhancement is unlikely to put CKM CP violation within reach of atomic EDM experiments, it may help to provide added sensitivity to non-SM CP violation. 
One advantage of the deuteron EDM experiment [263] is that it would involve deuteron ions rather than neutral atoms and, thus, evade the complications due to Schiff screening and provide a direct measurement. Recently, Ref. [289] suggested that $d_{D}$ may be enhanced in the chiral limit. $d_{D}$ was computed using a $\pi$ exchange model for the P-and T-odd $N N$ interaction leading to the result,

$$
d_{D}=-\frac{e g_{A} g_{1}}{3 m_{\pi}}\left(\frac{m_{N}}{\Lambda_{\chi}}\right) \frac{1+\xi}{(1+2 \xi)^{2}} \approx 2.5 \times 10^{-14} g_{1} e-\mathrm{cm},
$$

where $g_{1}$ gives the strength of the $\Delta I=1$, P-and T-odd pion-nucleon coupling,

$$
\mathcal{L}_{\Delta I=1}^{\mathrm{P}, \mathrm{T}}=g_{1}(\bar{p} p+\bar{n} n) \pi^{0}
$$

and where $\xi=\sqrt{m_{N} E_{B}} / m_{\pi}$ ( $E_{B}$ is the deuteron binding energy). Thus, a measurement of $d_{D}$ at the $10^{-27} e$-cm level would be sensitive to a $g_{1}$ of $\mathcal{O}\left(10^{-13}\right)$. A non-zero value for $g_{1}$ in the SM - either due to CKM CP violation or $\mathcal{L}_{\text {strong } \mathrm{CP}}$ - is suppressed, so it can be fairly sensitive to CP violation in models for physics beyond the SM.

Given that the CKM induced EDMs of all systems under study are expected to be far smaller in magnitude than present or prospective experimental sensitivities, the EDM measurements are primarily searches for either strong CP violation or CP violation that goes beyond the SM. In order to derive implications for the latter, one must, however, determine the level at which strong $\mathrm{CP}$ violation contributes to any EDM. In this respect, a comparison of various experiments is essential. In particular, both atomic and neutron EDMs are sensitive to $\theta_{\mathrm{QCD}}$, whereas the lepton and deuteron EDM sensitivity to strong CP violation is highly suppressed. In the case of both $d_{n}$ and $d_{A}$, the dominant effect of $\theta_{\mathrm{QCD}}$ arises via an induced, $\Delta I=0, \mathrm{~T}$ - and P-odd $\pi N N$ interaction,

$$
\mathcal{L}_{\Delta I=0}^{\mathrm{P}, \mathrm{T}}=g_{0} \bar{N} \vec{\tau} \cdot \vec{\pi} N
$$

where the authors of Ref. [290] estimate,

$$
g_{0}=-\theta_{\mathrm{QCD}}\left[\frac{\left(m_{\Xi}-m_{N}\right) m_{u} m_{d}}{F_{\pi}\left(m_{u}+m_{d}\right)\left(2 m_{s}-m_{u}-m_{d}\right)}\right] \approx-0.4 \theta_{\mathrm{QCD}} .
$$

The resulting neutron EDM is singular in the chiral limit,

$$
d_{n}=\frac{g_{A} g_{0}}{\Lambda_{\chi} \pi} \ln \frac{\Lambda_{\chi}}{m_{\pi}}+\cdots
$$

where the dots indicate subleading contributions. (For other related theoretical discussions, see, e.g., Ref. 291.) The corresponding dependence of the atomic EDM on $\theta_{\mathrm{QCD}}$ does not have a simple analytic expression, since it depends on a complex interplay of nuclear and atomic structure computations. Nonetheless, the present limit on $d_{A}\left({ }^{199} \mathrm{Hg}\right)$ leads to the most stringent bound on strong CP violation,

$$
\theta_{\mathrm{QCD}}<1.5 \times 10^{-10} \quad(95 \% \mathrm{CL})
$$

while the present limit from the neutron EDM lead to a bound that is four times weaker.

Should a future more precise atomic or neutron EDM measurement yield a non-zero result, one would not be able to determine if the source was $\theta_{\mathrm{QCD}}$ or new $\mathrm{CP}$ violation without information from the lepton or deuteron experiments. Should one of the latter also observe a non-zero EDM, one would likely conclude that one had seen evidence for new CP violation. Similarly, should future atomic and neutron EDM measurements with comparable sensitivities to $\theta_{\mathrm{QCD}}$ obtain significantly different results that could not be attributed to hadronic or nuclear structure uncertainties, one would also favor an explanation in terms of $\mathrm{CP}$ violation beyond the SM. 
Although free from the effects of $\mathcal{L}_{\text {strong }}$ CP, the lepton and deuteron EDM measurements by themselves would not be sufficient to distinguish among various scenarios for new CP violation, should any of them find a non-zero result. However, the sensitivities of atomic and neutron EDMs to these scenarios are sufficiently complementary to those of lepton EDMs that, taken together, the full set of EDM studies provides a powerful diagnostic tool for new $\mathrm{CP}$ violation.

To illustrate, we consider the various EDMs in the context of the MSSM. In the case of the electron, for example, one has [277,

$$
d_{e}=-e \frac{\alpha}{4 \pi} M_{\tilde{\gamma}} \sin 2 \theta_{L R} \sin \left(\phi_{A}-\phi_{\tilde{\gamma}}\right) F\left(m_{\tilde{e}_{i}}, M_{\tilde{\gamma}}\right)
$$

when the EDM is dominated by graphs involving selectron $(\tilde{e})$-photino $(\tilde{\gamma})$ intermediate states. Here, $\theta_{L R}$ is the angle describing the mixing of chiral electron eigenstates into mass eigenstates with masses, $m_{\tilde{e}_{i}} ; F$ is a calculable function of these masses and that of the photino, $M_{\tilde{\gamma}} ;$ and $\phi_{A, \tilde{\gamma}}$ are CP violating phases. $\phi_{A}$ is associated with tri-scalar couplings that arise in the soft SUSY breaking Lagrangian, while $\phi_{\tilde{\gamma}}$ appears in Majorana mass terms for the neutral gauginos. Note that $d_{e}$ depends on the difference of these phases. For superpartner masses of order the weak scale one has,

$$
d_{e} \approx-\left(1 \times 10^{-25}\right)\left(\frac{M_{\tilde{\gamma}}}{100 \mathrm{GeV}}\right)^{-3}\left(\frac{\left|A_{e}\right|}{100 \mathrm{GeV}}\right) \sin \left(\phi_{A}-\phi_{\tilde{\gamma}}\right) e-\mathrm{cm}
$$

Thus, for $\sin \left(\phi_{A}-\phi_{\tilde{\gamma}}\right)$ of order unity, one would require $M_{\tilde{\gamma}}>500 \mathrm{GeV}$ (for $\left|A_{e}\right| \sim 100 \mathrm{GeV}$ ) in order to evade the present limit. For significantly lighter gauginos one would need $\sin \left(\phi_{A}-\phi_{\tilde{\gamma}}\right)$ to be small. Present collider limits on $M_{\tilde{\gamma}}$ are rather weak, so either possibility is currently allowed by experiment. Even the light gaugino scenario does not imply the individual SUSY CP violating phases need be small, but rather that the phase difference be so. Thus, even within the MSSM, a single EDM measurement may not be sufficient to determine an individual CP phase.

Significantly more information may be obtained by comparing EDM experiments. As has been noted by several authors, the EDMs of different systems display different sensitivities to the CP violating phases in SUSY. A useful illustration of this complementarity occurs in the context of gravity mediated SUSY breaking, in which one has two CP violating phases, $\phi_{A}$ and $\phi_{\mu}$ associate with the $\mu$-term (see Section 1.4). This complementary phase dependence, along with the current constraints from $d_{e}, d_{n}$, and $d_{A}\left({ }^{199} \mathrm{Hg}\right)$ is illustrated in Figure 5 (see also Ref. [292]). When taken together, the EDM experiments imply that for light superpartners, $\phi_{A}$ and $\phi_{\mu}$ individually - and not just $\left|\phi_{A}-\phi_{\mu}\right|$ - must be small. This information provides important input for theorists who build models of SUSY breaking mediation, who must explain why these SUSY phases are small.

An alternative and more esoteric illustration of the EDM complementarity arises in the case of new interactions that violate $\mathrm{T}$ but conserve $\mathrm{P}$. In the presence of parity violating SM EW radiative corrections, these time-reversal violating, parity conserving (TVPC) interactions could give rise to EDMs of various particles 293. Since the only TVPC interactions one can write down are non-renormalizable, their impact on EDMs in the presence of radiative corrections must be treated carefully in the context of an effective field theory (EFT) [294 295]. As noted in Ref. [295], EDMs provide unambiguous constraints on TVPC interactions only when one assumes that the scale at which $\mathrm{P}$ is broken is well below that of the breakdown of $\mathrm{T}$. Then the lepton and neutron EDMs are considerably more sensitive to these effects than the EDMs of neutral atoms such as ${ }^{199} \mathrm{Hg}$ [296]. Thus, were one to observe non-vanishing EDMs in leptons and the neutron but not in neutral atoms, one might favor an explanation in terms of TVPC interactions. This possibility contrasts with that of strong $\mathrm{CP}$ violation that allows for non-vanishing neutron and atomic but not leptonic EDMs. SUSY would naturally imply non-vanishing EDMs for all three systems at comparable levels. 
Combination of $d_{n}, d_{e}$ and $d_{H g}$

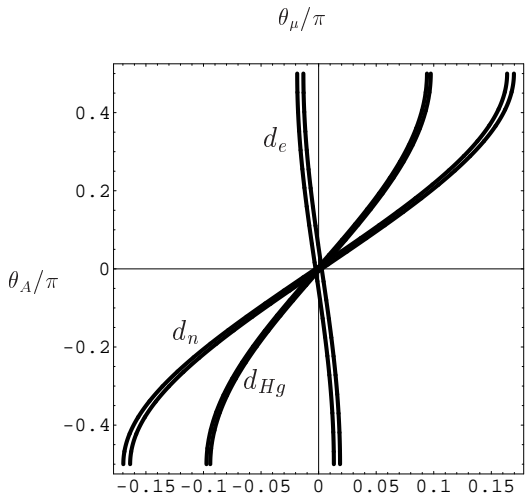

$M=250 \mathrm{GeV}$

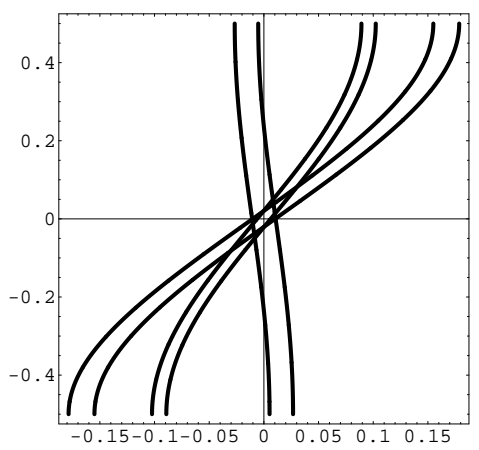

$M=500 \mathrm{GeV}$

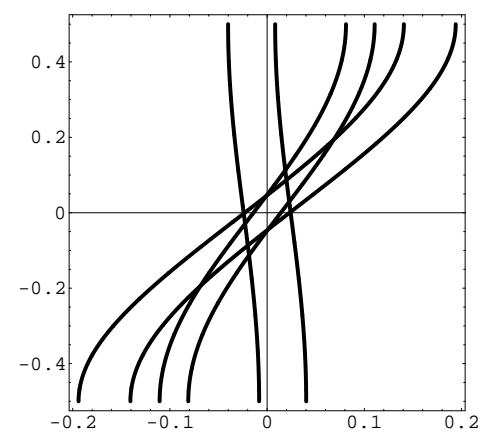

$M=750 \mathrm{GeV}$

Figure 5: Constraints on CP violating supersymmetric phases from electron, neutron, and ${ }^{199} \mathrm{Hg}$ EDM limits. They are shown assuming three different values of a common superpartner mass and $\tan \beta=3$. (Figure provided by M. Pospelov.)

\subsubsection{EDMs and the baryon asymmetry of the Universe}

One of the strongest theoretical motivations for postulating the existence of CP violation beyond the CKM and strong CP violation of the SM, is the need to account for the predominance of matter over antimatter in the Universe. As observed by Sakharov in 1967 [297], if the initial conditions of the Universe were exactly matter-antimatter symmetric, then during the subsequent evolution of the Universe three discrete symmetries would have to be violated in order to explain the observed baryon asymmetry: baryon number (B), charge conjugation, and CP. Assuming CPT is an exact symmetry, these other symmetry violations also need to be accompanied by a departure from thermal equilibrium, such as a first order phase transition. Many attempts to account for the BAU have focused on the EW phase transition and the possibilities for new weak scale CP violation. Presently, a popular alternative to such electroweak baryogenesis (EWB) involves CP violation in the lepton sector associated with neutrino masses and mixing (leptogenesis). Typically, the latter $\mathrm{CP}$ violation is associated with very 
high scales, as in see-saw models of neutrino mass 248 250] (see Section 5.1). The effects on EDMs of leptonic CP violation are expected to fall well below those in the SM [298], so we focus here on the connections between EDMs and EWB.

As noted above, the magnitude of CP violation in the SM is far too weak to account for the observed BAU. Moreover, numerical studies indicate that the strength of the first order SM EW phase transition is too weak to prevent subsequent wash out of sphaleron-induced baryon number creation. In contrast, models that contain new sources of CP violation, such as SUSY or theories with a more elaborate Higgs sector, appear to produce a sufficiently strong first order phase transition. Within the MSSM, the window of opportunity for satisfying this requirement has narrowed, as one needs a fairly light Higgs (see e.g. Ref. 299] and references therein). However, this requirement can be relaxed by extending the MSSM gauge group. Thus, SUSY EWB remains a viable option for producing the BAU.

Several authors have attempted to compute the BAU in the MSSM using a bubble wall picture of the phase transition (for a recent review and references, see Ref. [300]). In this scenario, a small region of broken EW symmetry (the bubble) expands into the unbroken vacuum, and the B-, C-, and CP violating processes needed for the BAU occur in the plasma at the interface between the two phases (the bubble wall). Efforts to model this plasma region have typically relied on various simplifying assumptions and approximations, and not all of the associated uncertainties have been adequately quantified. Nevertheless, these studies provide some guidance as to the implications of EDM measurements for EWB. For example, Ref. 301 finds for the ratio of baryon number density, $\rho_{B}$, to the entropy, $S$, at photon freeze-out,

$$
\frac{\rho_{B}}{S}=-\gamma_{w} v_{w}\left(\frac{\kappa}{\kappa^{\prime}}\right) \Delta \beta\left[6.3 \sin \phi_{\mu}+0.23 \sin \left(\phi_{A}-\phi_{\mu}\right)\right] \times 10^{-9}
$$

where $\gamma_{w} v_{w}$ is the velocity of the bubble wall, $\kappa / \kappa^{\prime}$ gives the ratio of weak and strong sphaleron rates, and $\Delta \beta$ characterizes the change in $\tan \beta$ (see Section 1.4) across the plasma. The first term in Eq. (119) arises from $\mathrm{CP}$ violating interactions between charginos and neutralinos and the spacetime dependent Higgs VEVs while the second term is generated by Higgs VEV-scalar top quark interactions. In arriving at this result, Ref. 301 assumed fairly light gaugino and squark masses. The relative weighting of the two terms in Eq. (119) will differ under other assumptions for the MSSM parameters. From the dominance of the first term and the experimental range of $(4-7) \times 10^{-11}$ for $\rho_{B} / s$, one infers 301 $\left|\sin \phi_{\mu}\right| \geq 0.025$. The constraints from the EDMs shown in Figure 5 imply $\left|\sin \phi_{\mu}\right| \lesssim 0.03$, a limit that is barely consistent with the requirements of the BAU. In order for the interactions involving squarks to play an appreciable role in the BAU, $\left|\sin \left(\phi_{A}-\phi_{\mu}\right)\right|$ must be considerably larger than allowed by the EDM constraints. One might concludes that existing EDM measurements have severely constrained the viability of supersymmetric EWB - even with unnaturally small $\mathrm{CP}$ violating phases — and that future measurements will have an even more substantial impact on this scenario.

The extent to which theoretical uncertainties in the analysis leading to Eq. (119) and the EDM limits of Figure 5]allow for the MSSM to evade these conclusions is a topic of on-going study. Ref. [302], for example, has argued that memory effects in the plasma may significantly enhance the impact of CP violating interactions, allowing for the production of the observed BAU with considerably smaller CP violating phases. If these arguments survive further scrutiny, then the present EDM constraints would not substantially limit the viability of EWB in the MSSM, while the impact of future, more precise measurements would have to be analyzed in more detail.

\subsection{Lepton Flavor Violation}

The observation of neutrino oscillations has inspired renewed interest in the more general question of lepton flavor non-conservation. Experimentally, the most powerful probes of lepton flavor violation (LFV) involve decays of the muon, such as $\mu \rightarrow e \gamma, \mu+A(Z, N) \rightarrow e+A(Z, N)$, or $\mu \rightarrow 3 e$. The most 
stringent limit on the process $\mu \rightarrow e \gamma$ has been obtained by the MEGA Collaboration [303],

$$
\mathcal{B}(\mu \rightarrow e \gamma) \equiv \frac{\Gamma\left(\mu^{+} \rightarrow e^{+} \gamma\right)}{\Gamma\left(\mu^{+} \rightarrow e^{+} \nu \bar{\nu}\right)}<1.2 \times 10^{-11}(90 \% \mathrm{CL}),
$$

while the SINDRUM Collaboration [304] has obtained a limit on the conversion branching ratio,

$$
R_{\mu \rightarrow e}^{A} \equiv \frac{\Gamma\left[\mu^{-}+A(N, Z) \rightarrow e^{-}+A(N, Z)\right]}{\Gamma\left[\mu^{-}+A(Z, N) \rightarrow \nu_{\mu} A(Z-1, N+1)\right]}<6.1 \times 10^{-13}(90 \% \mathrm{CL}),
$$

for conversion in $\mathrm{Au}$. The SINDRUM experiment has also constrained the $\mu \rightarrow 3 e$ branching ratio to be less than $1 \times 10^{-12}(90 \% \mathrm{CL})$ 305, and the branching ratio for the lepton number violating conversion process in $\mu^{-} \rightarrow e^{+}$in Ti is $<3.6 \times 10^{-11}(90 \% \mathrm{CL})$ [306]. A new experiment at PSI hopes to improve on the MEGA sensitivity by three orders of magnitude [307, while the MECO experiment at Brookhaven expects to achieve a four orders of magnitude improvement 308.

The more precise LFV searches are poised to probe NP above the EW scale. Although flavor oscillations among light neutrinos will induce LFV decays of charged leptons at some level, the corresponding rates are typically suppressed by factors of $\left(\Delta m_{\nu}^{2} / M_{W}^{2}\right)^{2} \sim 10^{-50}$ and completely negligible. In contrast, flavor violation involving heavy particles that are not part of the SM may generate effects that could be seen by experiment. In this respect, two broad scenarios have been studied theoretically [309 318].

A commonly quoted model involves GUT scale LFV and supersymmetry. GUTs naturally provide a link between flavor violation in the quark and lepton sectors 312313 . In this context, LFV occurs at the GUT scale via Yukawa couplings involving quark and lepton supermultiplets. The assignment of leptons and quarks to the same representation of the unification group implies that the large Yukawa coupling responsible for the top quark mass also appears in the LFV terms, thereby leading to unsuppressed LFV couplings. At the weak scale, these can be related in a straightforward way to the CKM matrix elements for the top quark, modulo RG evolution from the GUT scale to the weak scale. To avoid GIM [319] suppression by the light neutrino mass differences or by inverse powers of the GUT scale, the lepton flavor mixing effects must be realized in a sector associated with $\mathrm{TeV}$ (or lower) scales. Supersymmetry provides a natural mechanism for doing so, as the GUT induced couplings between quark and lepton supermultiplets can give rise to lepton flavor non-diagonal terms in the soft SUSY breaking interactions. Superpartner loops that include insertions of the LFV operators can then generate unsuppressed contributions to $\mu \rightarrow$ er, etc.

For example, in a SUSY $S U(5)$ scenario one has 312,

$$
\mathcal{B}(\mu \rightarrow e \gamma)=2.4 \times 10^{-12}\left(\frac{\left|V_{t s}\right|}{(0.04)} \frac{\left|V_{t d}\right|}{(0.01)}\right)^{2}\left(\frac{100 \mathrm{GeV}}{m_{\tilde{\mu}}}\right)^{4}
$$

where gaugino mass effects have been neglected for illustrative purposes. One expects $R_{\mu \rightarrow e}^{\mathrm{Ti}}$ to be smaller than $\mathcal{B}(\mu \rightarrow e \gamma)$ by a factor of about $2.4 \alpha$, since a virtual photon is exchanged between the leptons and nucleus instead of a real photon being emitted. The $\mathcal{O}(\alpha)$ suppression of $R_{\mu \rightarrow e}^{A}$ relative to $\mathcal{B}(\mu \rightarrow e \gamma)$ is a rather generic feature of GUT scale LFV. In both cases, the GUT expectation is at least an order of magnitude below the current experimental bounds.

An alternate scenario involves the generation of LFV at the TeV scale [309 315]. This scenario can also be realized in the MSSM, though it does not arise naturally from any conventional model of SUSY breaking mediation. The latter generally entail flavor universality at the SUSY breaking scale, so that some alternate mechanism for LFV, such as GUT scale lepton-quark unification or RPV [316] is needed. In contrast, if a GUT breaks down in stages to the SM gauge group (11), then LFV Yukawa interactions can involve particles with intermediate scale masses.

One may consider, e.g., a GUT that breaks down to the group $S U(3)_{C} \times S U(2)_{L} \times S U(2)_{R} \times U(1)_{B-L}$ of left-right symmetric models. If the right-handed gauge symmetry is broken at a the TeV scale, 
interactions involving right-handed (Majorana) neutrinos, gauge bosons, and Higgs multiplets may give rise to unsuppressed LFV. In contrast to the GUT scale LFV scenario, the presence of relatively light Higgs multiplets may lead to a substantial enhancement of $R_{\mu \rightarrow e}^{A}$, making it comparable in magnitude to $\mathcal{B}(\mu \rightarrow e \gamma)$ 317318. Specifically, one finds an enhancement by the square of a large logarithm involving the $S U(2)_{L} \times S U(2)_{R}$ breaking scale and $m_{\mu}$ [318, compensating for the $\mathcal{O}(\alpha)$ suppression of $R_{\mu \rightarrow e}^{A}$ relative to $\mathcal{B}(\mu \rightarrow e \gamma)$. One therefore expects in this scenario the two LFV ratios to be of comparable magnitude. Similar logarithmic enhancements may occur in RPV SUSY scenarios [316].

\section{$5 \quad$ Lepton Properties}

The observation of neutrino oscillations in atmospheric, solar, and reactor neutrino experiments has provided the first incontrovertible evidence for physics beyond the SM. Although a thorough review of neutrino phenomenology merits an entire article in itself (see, e.g., the recent review by McKeown and Vogel [320] and references therein), no review of low energy weak interactions would be complete without some discussion of neutrinos. Below, we provide a short summary of neutrino properties, with an emphasis on experimental results. In brief, the picture that has emerged from experiment implies substantial flavor mixing among the three lightest neutrinos, in contrast to the situation involving quarks. Labeling the neutrino mass eigenstates as $\nu_{i}(i=1,2,3)$ with masses $m_{i}$, one finds tiny masssquared differences, $\left|m_{3}^{2}-m_{2}^{2}\right| \approx 0.002 \mathrm{eV}^{2}$ from atmospheric oscillations and $\left|m_{2}^{2}-m_{1}^{2}\right| \approx 10^{-5} \mathrm{eV}^{2}$ from solar neutrinos. The absolute scale of neutrino mass is not yet known, but upper bounds exist from various $\beta$-decay studies (see below) as well as cosmological observations $\left(\sum_{i} m_{i}<0.7 \mathrm{eV}\right.$ for neutrinos light enough to decouple while still relativistc [321]). A variety of neutrino properties remain to be determined by additional experimental work:

- The charge conjugation properties of the neutrino, i.e., whether it is a Dirac or Majorana particle.

- The nature of the neutrino mass hierarchy, namely whether it is degenerate $\left(\left|\Delta m_{i j}^{2}\right| \ll m_{j}^{2}\right.$ for all $i, j)$ or non-degenerate and whether it is "normal" $\left(m_{1}^{2}<m_{2}^{2}<m_{3}^{2}\right)$ or inverted.

- The size of the mixing angle $\theta_{13}$ and the $\mathrm{CP}$ violating Dirac phase $\delta$, as well as the two $\mathrm{CP}$ violating Majorana phases if neutrinos are Majorana particles.

In addition, the LSND experiment, which implies a much larger $\Delta m^{2}$ than obtained from either the atmospheric or solar oscillation experiments, has raised the possibility that there exists either a fourth generation of light neutrinos or of CPT violation among the three generations.

The origins of neutrino masses and mixing, as well as the properties of neutrinos with respect to various discrete symmetries, have inspired a wealth of theoretical proposals for physics beyond the SM. Since a review of these ideas goes beyond the scope of this article, we content ourselves with brief statements about the simplest ones, along with a compilation of results from the various experiments.

\subsection{Neutrino masses and mixing}

In the minimal (without left-handed $\bar{\nu}$ ) renormalizable SM as described by Eq. (12) neutrinos are predicted to be massless. In particular, Majorana mass terms [322] for the left-handed neutrinos,

$$
\mathcal{L}_{\text {Majorana }}=-\frac{m_{M}}{2} \bar{\nu} C \bar{\nu}^{T}
$$

where $C$ is the charge-conjugation matrix defined by $C \gamma_{\mu} C^{-1}=-\gamma_{\mu}^{T}$, are forbidden by $U(1)_{Y}$ invariance and not produced upon EW symmetry breaking. The simplest way to produce a term of the form (123) 
is to add a Higgs triplet 323 carrying one unit of hypercharge ${ }^{14}$. However, unless one has a minuscule Yukawa coupling, one would expect ${ }^{15} m_{M}=\mathcal{O}(10 \mathrm{GeV})$. Note that the mass term (123) violates the conservation of any additive quantum number that $\nu$ may carry, such as lepton number. This can lead to neutrinoless double $\beta$ decay as will be discussed in Subsection 5.6 .

Alternatively, the introduction of a left-handed anti-neutrino, $\bar{\nu}$, would allow a Dirac mass term,

$$
\mathcal{L}_{\text {Dirac }}=-m_{D} \bar{\nu} \nu
$$

in complete analogy to Eqs. (10 1112). Thus, $\nu$ and $\bar{\nu}$ combine to yield a Dirac neutrino with four physically distinct degrees of freedom. As before, one generally expects $m_{D}=\mathcal{O}\left(G_{F}^{-1 / 2}\right)$. If there is more than one Dirac neutrino one expects CKM mixing analogous to Eq. (14) 325]. Thus, while lepton number is conserved in the Dirac case, lepton flavor number associated with a particular lepton generation is not.

In the case of $n$ Majorana neutrinos, $m_{M}$ becomes in general a (complex) symmetric $n \times n$ mass matrix. Each neutrino spinor may absorb an unphysical phase, reducing $m_{M}$ to $n^{2}$ real observable parameters, namely $n$ mass eigenvalues, $n(n-1) / 2$ mixing angles, and an equal number of CP violating phases. A Dirac neutrino corresponds to the special case of a pair of Majorana neutrinos of equal mass and maximal mixing from their weak interaction basis. A pure Majorana neutrino corresponds to no mixing. In the most general situation, both types of mass terms may be present. Another special case arises for $n=2$ and $m_{M} \ll m_{D}$, i.e., the diagonal (Majorana mass) entries are much smaller than the off-diagonal (Dirac mass) term. This is called a pseudo-Dirac neutrino. On the other hand, if one Majorana mass entry vanishes and the other one satisfies $m_{M} \gg m_{D}$, one obtains eigenvalues of order $m_{M}$ and $m_{D}^{2} / m_{M}$, referred to as the see-saw mechanism 248 250. Thus, if the see-saw mechanism is realized in nature, very small neutrino masses would probe very high mass scales, $m_{M}$. More generally a fundamental theory beyond the SM will produce non-renormalizable terms in the Lagrangian carrying coefficients of negative mass dimensions which are numerically suppressed by the NP scale and could therefore naturally produce small neutrino masses. The simplest possibility is a dimension five term involving two lepton doublets and two Higgs doublets [326].

Neutrino masses can reveal themselves by a variety of effects. The most important classes are kinematic effects in decays, neutrino oscillations, and possible effects related to the now allowed magnetic and electric dipole moments.

The most precise kinematic limits are obtained from two tritium $\beta$ decay experiments [327328]. Assuming there are no common uncertainties the can be combined yielding,

$$
m_{\bar{\nu}_{e}}^{2}(\text { eff. }) \equiv \sum_{i}\left|U_{e i}\right|^{2} m_{\nu_{i}}^{2}=-2.5 \pm 3.3 \mathrm{eV}^{2}
$$

where $U$ is the neutrino mixing matrix, and the sum is over all mass eigenvalues, $m_{\nu_{i}}$, that cannot be resolved experimentally ${ }^{16}$. More than $77 \%$ of the range (125) is in the unphysical (spontaneously Lorentz invariance breaking) region ${ }^{17}$ with $m_{\nu_{e}}^{2}$ (eff.) $<0$. Normalizing the probability conditional on $m_{\nu_{e}}^{2}$ (eff.) $>0$ yields the $95 \%$ CL upper limit,

$$
\sqrt{m_{\bar{\nu}_{e}}^{2}(\text { eff. })}<2.3 \mathrm{eV}
$$

\footnotetext{
${ }^{14}$ A Higgs singlet could also produce a Majorana mass term, but if it does it necessarily breaks $U(1)_{\mathrm{EM}}$.

${ }^{15}$ Triplet VEVs are severely constrained by EW precision data and can be at most of order $10 \mathrm{GeV}$ [12. Values considerably smaller than that would require fine-tuning in the Higgs potential order by order in perturbation theory [324.

${ }^{16}$ See Ref. 329] for a discussion.

${ }^{17}$ There are several other tritium $\beta$ decay experiments some of which yielding results which are incompatible with $m_{\nu_{e}}^{2}$ (eff.) $>0$. An event excess near the spectrum endpoint leading to an apparent $m_{\nu_{e}}^{2}$ (eff.) $<0$ is presently not understood, but Refs. 327328 apply corrections to obtain results that can be properly interpreted.
} 
The KATRIN Collaboration [330] at the Forschungszentrum in Karlsruhe, Germany, will attempt to improve this limit in a next-generation tritium $\beta$ decay experiment to the $0.35 \mathrm{eV}$ level.

Limits analogous to (125) can also be obtained for $\nu_{\mu}$ (from $\pi^{ \pm}$decays) and for $\nu_{\tau}$ (from $\tau^{ \pm}$decays). However, these limits are many orders of magnitude weaker and relevant only to the extent to which the positive results on neutrino mass differences from neutrino oscillations are circumvented. For a recent review on absolute neutrino masses, see Ref. 331.

The weak interaction induces one-loop neutrino magnetic moments proportional to the neutrino masses. However, given the limit (125) the SM contribution to the magnetic moment is many orders of magnitude below current limits. Thus, the search for magnetic and (CP violating) electric dipole moments of neutrinos provides tests of physics beyond the SM with massive neutrinos.

If for a given pair of weak eigenstate neutrinos, $\nu_{\alpha}$ and $\nu_{\beta}$, (i) one or both neutrinos are massive, (ii) the mass eigenvalues are not identical, $m_{\nu_{\alpha}} \neq m_{\nu_{\beta}}$, and (iii) the mass eigenstates differ from the weak interaction eigenstates by a mixing angle, $\theta, \nu_{\alpha}=\cos \theta \nu_{1}+\sin \theta \nu_{2}$ (where $\cos \theta \neq 1$ ), one predicts the phenomenon of neutrino oscillations 332 338]. For highly relativistic, $m_{i} \ll E_{i}$, and monochromatic neutrinos one finds the probability for oscillations in vacuum,

$$
P\left(\nu_{\alpha} \rightarrow \nu_{\beta}\right)=1-\left|<\nu_{\alpha}(0)\right| \nu_{\alpha}(t)>\left.\right|^{2}=\sin ^{2} 2 \theta \sin ^{2}\left[\frac{\left(m_{1}^{2}-m_{2}^{2}\right) t}{4 E}\right] \approx \sin ^{2} 2 \theta \sin ^{2}\left[1.267 \Delta m^{2} \frac{L}{E}\right]
$$

where the second form assumes that the masses are given in $\mathrm{eV}$, the distance, $L \approx t$, traveled from production to detection in $\mathrm{km}$, and the neutrino energies, $E \approx E_{1} \approx E_{2}$, in $\mathrm{GeV}$. It is assumed that both $L$ and $E$ are fixed and known with negligible uncertainty. Otherwise, one has to take appropriate averages. For example, one may have to correct for finite source or detector effects or non-monochromaticity. In the limit of very large oscillations, $P\left(\nu_{\alpha} \rightarrow \nu_{\beta}\right) \rightarrow \sin ^{2}(2 \theta) / 2$. Quantities of the form $P\left(\nu_{\alpha} \rightarrow \nu_{\beta}\right)$ are measured in what are called $\nu_{\beta}$ appearance experiments, while $P\left(\nu_{\alpha} \rightarrow \nu_{\alpha}\right)$ is the object of interest in $\nu_{\alpha}$ disappearance experiments.

This formalism is easily extended to multi-flavor oscillations, where the three standard neutrinos (even if their masses are of Dirac type) will in general produce CP violation in the lepton sector. These flavor oscillations are also called first class. If one wishes to introduce a fourth neutrino, this would almost certainly be sterile (see Section [1.2), as the number of active neutrinos, $N_{\nu}=2.986 \pm 0.007$, with $m_{\nu} \ll M_{Z} / 2$ is strongly constrained by LEP (see Section [1.3). Allowing $m_{\nu} \gtrsim M_{Z} / 2$ to avoid this would either require the introduction of a whole set of fermions (such as a fourth generation) to cancel gauge anomalies which in turn (due to chiral non-decoupling effects) would clash with EW precision data; or would require a vector-like pair of lepton doublets. In any case, the experimental hints and signals to be discussed in the Subsections below, all involve very small $\Delta m^{2}$, so that the LEP bound on active neutrinos holds. Oscillations of ordinary into sterile neutrinos [339], $\nu_{s}$, are called second class.

To simplify the discussion and to facilitate the comparison between different experiments we will always assume simple two-neutrino oscillation scenarios. We primarily summarize the experiments yielding the most stringent limits [340].

\subsection{Solar Neutrinos}

Detectors observing solar neutrinos fall into two categories ${ }^{18}$. Radiochemical detectors $(\mathrm{Cl}$ or $\mathrm{Ga})$ are blind to any spectral or directional information, and time variation studies are limited by the extraction periods of typically several weeks. But they have the great advantage of relatively low neutrino energy thresholds set by the $\mathrm{CC}$ reactions,

$$
\nu_{e}+{ }^{37} \mathrm{Cl} \rightarrow e^{-}+{ }^{37} \mathrm{Ar}, \quad \nu_{e}+{ }^{71} \mathrm{Ga} \rightarrow e^{-}+{ }^{71} \mathrm{Ge} .
$$

\footnotetext{
${ }^{18}$ For a recent overview of solar neutrino experiments, see Refs. 341342.
} 
Cherenkov detectors (light or heavy water) are only sensitive to the highest energy neutrinos, but are real time experiments. In addition, energy and direction of the incident neutrino can be estimated, and there is a $\mathrm{NC}$ component in the basic electron scattering (ES) process,

$$
\nu+e^{-} \rightarrow \nu+e^{-}
$$

\subsubsection{Rates}

The experimental results of the capture rates in the radiochemical experiments and of the neutrino fluxes in the Cherenkov detectors are compared to predictions based on the Standard Solar Model (SSM). We use here the latest available results [343] (updated from Ref. 344]) which include the recent measurement 345] of the low energy Beryllium-proton fusion cross section ${ }^{19}$ Experiments using Cherenkov detectors quote results assuming no neutrino oscillations and an undistorted neutrino spectrum, where the latter has been verified experimentally.

The $\mathrm{Cl}$ detector in the Homestake mine in South Dakota represented the first solar neutrino experiment and also the one with the longest running time (1970-1994). The combined result of 108 extractions of solar induced ${ }^{37} \mathrm{Ar}$ is 348 , ( $1 \mathrm{SNU}=1$ interaction per second and $10^{36}$ target atoms),

$$
\left.2.56 \pm 0.16 \text { (stat.) } \pm 0.16 \text { (syst.) SNU } \quad \text { SSM: } 8.59_{-1.2}^{+1.1} \mathrm{SNU}\right]
$$

Thus, the ratio of the observed to expected capture rates,

$$
R\left({ }^{37} \mathrm{Cl}\right)=0.298_{-0.046}^{+0.049}
$$

corresponds to a deficit ${ }^{20}$ of $7.3 \sigma$. The SSM also estimates that almost $79 \%$ of the neutrinos with energies above the $\mathrm{Cl}$ threshold of $0.814 \mathrm{MeV}$ originate from ${ }^{8} \mathrm{~B}$ disintegration, and more than $13 \%$ from ${ }^{7}$ Be electron capture.

The deep underground SAGE experiment at the Baksan Neutrino Observatory in Russia was the first $\mathrm{Ga}$ experiment to take data and is still on-going. The advantage of $\mathrm{Ga}$ over $\mathrm{Cl}$ is the lower neutrino threshold of only $0.233 \mathrm{MeV}$ allowing the detection of the dominant proton-proton fusion neutrinos (pp), which contribute about $54 \%$ of the flux, with ${ }^{7} \mathrm{Be}(26 \%)$ and ${ }^{8} \mathrm{~B}(11 \%)$ subleading. Recently, the SAGE Collaboration presented results [349] of their first 12 years (92 runs) of observation from January 1990 through December 2001,

$$
\left.70.8_{-5.2}^{+5.3} \text { (stat. }\right)_{-3.2}^{+3.7} \text { (syst.) SNU. }
$$

A similar experiment with the GALLEX detector at the Gran Sasso Underground Laboratories collected a total of 65 runs from May 1991 through January 1997. The GALLEX Collaboration found a capture rate [350],

$$
77.5 \pm 6.2 \text { (stat.) })_{-4.7}^{+4.3} \text { (syst.) SNU, }
$$

in agreement with SAGE. Subsequently, GALLEX was succeeded by GNO, an upgraded and improved Ga experiment. The first published capture rate by the GNO Collaboration [351],

$$
65.8_{-9.6}^{+10.2} \text { (stat.) }{ }_{-3.6}^{+3.4} \text { (syst.) SNU, }
$$

\footnotetext{
${ }^{19}$ The cross section quoted in Ref. 345] is larger (but consistent) and more precise than the one quoted in Ref. 346] which was employed in Ref. 344. This drives the SSM predictions for all fluxes and capture rates up. The most recent result by the ISOLDE Collaboration [347] is slightly lower, of the same precision, and consistent within $1.1 \sigma$ when compared to the one in Ref. [345] (which is, however, currently under revision [347]).

${ }^{20}$ The uncertainties are mainly fractional, so that it is more appropriate to assume an (asymmetric) log-normal rather than a normal distribution. Note also that Gaussian error distributions are not well defined for positive-definite quantities like capture rates or ratios thereof.
} 
is based on 19 runs between May 1998 and January 2000 and consistent with both SAGE and GALLEX. The combined result of GALLEX and the first GNO period is 351,

$$
74.1 \pm 5.4 \text { (stat.) })_{-4.2}^{+4.0} \text { (syst.) SNU. }
$$

Finally, we combine the results (132) and (135) by (conservatively) assuming the smaller systematic uncertainty (SAGE) as common to SAGE and the Gran Sasso experiments. We obtain a total observed Ga capture rate,

$$
72.2_{-5.1}^{+5.3} \mathrm{SNU} \quad\left[\mathrm{SSM}: 130_{-7}^{+9} \mathrm{SNU}\right] .
$$

and for the ratio of the observed to expected capture rates,

$$
R\left({ }^{71} \mathrm{Ga}\right)=0.556_{-0.055}^{+0.051},
$$

corresponding to a deficit ${ }^{20}$ of $6.4 \sigma$. Thus, both types of radiochemical experiments see very large and statistically significant deficits. Moreover, taking the ratio of the ratios (131) and (137) shows at the $3.2 \sigma$ level (or more, considering that the uncertainties from the SSM partly cancel in the double ratio) that the deficits do not correspond to an overall reduction.

The Kamiokande water Čherenkov detector in the Kamioka mine in Japan studied solar neutrinos via electron scattering (129) from January 1987 to February 1995. The recoil electron energy threshold was chosen relatively high to avoid large backgrounds and varied between 7.0 and 9.3 MeV. This implies sensitivity almost exclusively to the ${ }^{8} \mathrm{~B}$ neutrino flux, $\Phi\left({ }^{8} \mathrm{~B}\right)$, including a few per mille neutrino contribution from Helium-proton (hep) fusion ${ }^{21}$. The Kamiokande Collaboration found the flux [352,

$$
\Phi\left({ }^{8} \mathrm{~B}\right)=2.80 \pm 0.19 \text { (stat.) } \pm 0.33 \text { (syst.) } \times 10^{6} \mathrm{~cm}^{-2} \mathrm{~s}^{-1} \text {. }
$$

Subsequently, the Kamiokande detector was succeeded by the Super-Kamiokande upgrade including an increase in fiducial volume by a factor of 33. The first phase (Super-Kamiokande-I [353]) had an electron energy threshold of $5 \mathrm{MeV}$ and took place from May 1996 through July 2001. It resulted in a flux measurement 354,

$$
\Phi\left({ }^{8} \mathrm{~B}\right)=2.35 \pm 0.02 \text { (stat.) } \pm 0.08 \text { (syst.) } \times 10^{6} \mathrm{~cm}^{-2} \mathrm{~s}^{-1}
$$

which is consistent with the flux (138).

SNO is a heavy water Cherenkov detector in the Creighton mine in Ontario, Canada. During the first phase of the experiment, from November 1999 to May 2001, only $\mathrm{D}_{2} \mathrm{O}$ was present in the detector. In the final analysis of the completed first phase an electron energy threshold identical to that of Super-Kamiokande was chosen. The SNO Collaboration reported [355] a ${ }^{8} \mathrm{~B}$ flux based on electron scattering (129),

$$
\Phi\left({ }^{8} \mathrm{~B}\right)=2.39_{-0.23}^{+0.24} \text { (stat.) } \pm 0.12 \text { (syst.) } \times 10^{6} \mathrm{~cm}^{-2} \mathrm{~s}^{-1},
$$

which is in good agreement with the Kamioka experiments. The different energy threshold ${ }^{22}$ at Kamiokande and the different liquid at SNO should imply negligible common systematics among the three experiments. Assuming this, we find the combined ${ }^{8} \mathrm{~B}$ flux from electron scattering,

$$
\Phi\left({ }^{8} \mathrm{~B} ; \mathrm{ES}\right)=2.37 \pm 0.08 \times 10^{6} \mathrm{~cm}^{-2} \mathrm{~s}^{-1} \quad\left[\mathrm{SSM}: 5.93_{-0.89}^{+0.83} \times 10^{6} \mathrm{~cm}^{-2} \mathrm{~s}^{-1}\right],
$$

and for the ratio of the observed to expected ${ }^{8} \mathrm{~B}$ fluxes,

$$
R\left({ }^{8} \mathrm{~B} ; \mathrm{ES}\right)=0.400_{-0.057}^{+0.061}
$$

\footnotetext{
${ }^{21}$ We will ignore hep neutrinos in the following as they have not been treated identically in the various papers.

${ }^{22}$ The different electron energy threshold may also have a small effect (which we ignore) on the NC contribution to the electron scattering cross section.
} 
again showing a $6.0 \sigma$ deficit $^{20}$. Unique to $\mathrm{SNO}$ is the ability to study $\mathrm{CC}$ and $\mathrm{NC}$ deuterium breakup,

$$
\nu_{e}+d \rightarrow e^{-}+p+p, \quad \nu+d \rightarrow \nu+p+n
$$

which in its first phase resulted, respectively, in flux measurements,

$$
\begin{aligned}
& \Phi\left({ }^{8} \mathrm{~B} ; \mathrm{CC}\right)=1.76_{-0.05}^{+0.06} \text { (stat.) } \pm 0.09 \text { (syst.) } \times 10^{6} \mathrm{~cm}^{-2} \mathrm{~s}^{-1}, \\
& \left.\Phi\left({ }^{8} \mathrm{~B} ; \mathrm{NC}\right)=5.09_{-0.43}^{+0.44} \text { (stat. }\right)_{-0.43}^{+0.46} \text { (syst.) } \times 10^{6} \mathrm{~cm}^{-2} \mathrm{~s}^{-1},
\end{aligned}
$$

and in ratios of observed to expected ${ }^{8} \mathrm{~B}$ fluxes,

$$
R\left({ }^{8} \mathrm{~B} ; \mathrm{CC}\right)=0.297_{-0.045}^{+0.048}, \quad R\left({ }^{8} \mathrm{~B} ; \mathrm{NC}\right)=0.86_{-0.16}^{+0.17} .
$$

The CC ratio which shows another $7.5 \sigma$ deficit is virtually identical to $R\left({ }^{37} \mathrm{Cl}\right)$ implying that ${ }^{7} \mathrm{Be}$ and ${ }^{8} \mathrm{~B}$ neutrino depletions are approximately equal. In contrast, the NC ratio is consistent with SSM expectations. After completion of the first phase of $\mathrm{SNO}, \mathrm{NaCl}$ was added to the detector which enhances the capture efficiency of the NC neutrons drastically. The third phase (scheduled for summer of 2003) will be characterized by the addition of ${ }^{3} \mathrm{He}$ proportional counter tubes as an alternative way to increase the $\mathrm{NC}$ efficiency.

Thus, there are not only statistically convincing neutrino deficits in all three $\mathrm{CC}$ channels $\left({ }^{37} \mathrm{Cl}\right.$, ${ }^{71} \mathrm{Ga}$, and ${ }^{8} \mathrm{~B}$ ), but there is also strong evidence (at the $\gtrsim 4 \sigma \mathrm{CL}$ ) that the low energy (mainly pp) neutrinos are suppressed less than ${ }^{7} \mathrm{Be}$ or ${ }^{8} \mathrm{~B}$ neutrinos. On the other hand, $R\left({ }^{8} \mathrm{~B} ; \mathrm{NC}\right)$ is consistent with SSM expectations implying that the solar neutrino flux is dominated by active neutrinos. There is redundancy in the data since $\Phi\left({ }^{8} \mathrm{~B} ; \mathrm{ES}\right)$ can be expressed in terms of $\Phi\left({ }^{8} \mathrm{~B} ; \mathrm{CC}\right)$ and $\Phi\left({ }^{8} \mathrm{~B} ; \mathrm{NC}\right)$,

$$
\Phi\left({ }^{8} \mathrm{~B} ; \mathrm{ES}\right)=(1-\epsilon) \Phi\left({ }^{8} \mathrm{~B} ; \mathrm{CC}\right)+\epsilon \Phi\left({ }^{8} \mathrm{~B} ; \mathrm{NC}\right),
$$

where $1 / \epsilon=6.48$ is the ratio of theoretical cross sections for $\nu_{e}$ relative to the sum of $\nu_{\mu}$ and $\nu_{\tau}$. The ratios (145) then imply, $\Phi\left({ }^{8} \mathrm{~B} ; \mathrm{ES}\right)=2.27_{-0.12}^{+0.13} \times 10^{6} \mathrm{~cm}^{-2} \mathrm{~s}^{-1}$, in perfect agreement with the measurement (141). Thus, flux measurements of solar neutrinos provide very strong evidence for $\left(\nu_{e} \rightarrow \nu_{\mu}, \nu_{\tau}\right)$ flavor oscillations with any component of oscillations into $\nu_{s}$ strongly constrained. The Super-Kamiokande Collaboration also sets limits [356] on conversions into $\bar{\nu}_{e}$.

Note that with the crucial SNO result, $\Phi\left({ }^{8} \mathrm{~B} ; \mathrm{NC}\right) \gg \Phi\left({ }^{8} \mathrm{~B} ; \mathrm{CC}\right)$, relation (146) fully explains the observation, $R\left({ }^{8} \mathrm{~B} ; \mathrm{ES}\right)>R\left({ }^{37} \mathrm{Cl}\right)$. Due to (i) a downward shift in $R\left({ }^{8} \mathrm{~B}\right.$; ES) (Super-Kamiokande and SNO relative to Kamiokande); (ii) an upward shift in $R\left({ }^{37} \mathrm{Cl}\right)$ (final relative to preliminary Homestake results); and (iii) an upward shift in the Beryllium-proton cross section ${ }^{17}$ (Ref. [345] relative to Ref. [346]), the current data do not favor strongest depletion for ${ }^{7}$ Be neutrinos (or even a vanishing ${ }^{7} \mathrm{Be}$ flux at the best fit 357-361]) as used to be the case in the past. A new experiment, BOREXINO [362], with the capability to settle the question of the ${ }^{7} \mathrm{Be}$ flux, is currently under construction at Gran Sasso. It is a (real time) liquid scintillator detector and with a threshold energy of only $250 \mathrm{keV}$, it combines the advantages of the radiochemical and Čherenkov type detectors. Completion of the construction phase is expected in 2003 (a counting test facility is already in operation).

\subsubsection{Time variations and spectral distortions}

Signal variations on various time scales can give additional hints as to what the origin of the solar neutrino deficit might be. The Homestake, Kamiokande, and SAGE experiments took data over time spans of the order of solar cycles. With the exception of a small and controversial hint at an anticorrelation of the capture rate in some of Homestake's preliminary results, there is no evidence for a solar neutrino flux correlation with sun spot activity. 
Super-Kamiokande finds a seasonal rate variation at the $2.5 \sigma \mathrm{CL}$, consistent with the expectation from the distance variation between the sun and the Earth.

Neither Kamiokande nor Super-Kamiokande reported a significant day-night electron scattering cross section asymmetry. For example, Super-Kamiokande quotes,

$$
A_{\mathrm{DN}}(\mathrm{ES}) \equiv 2 \frac{\sigma_{D}-\sigma_{N}}{\sigma_{D}+\sigma_{N}}=-0.021 \pm 0.020 \text { (stat.) }{ }_{-0.012}^{+0.013} \text { (syst.). }
$$

On the other hand, SNO finds (at the $2.2 \sigma \mathrm{CL}$ ) a stronger night time CC cross section [363],

$$
\begin{aligned}
& \left.A_{\mathrm{DN}}(\mathrm{ES})=0.174 \pm 0.195 \text { (stat.) }\right)_{-0.024}^{+0.022} \text { (syst.) } \\
& A_{\mathrm{DN}}(\mathrm{CC})=-0.140 \pm 0.063(\text { stat. })_{-0.015}^{+0.014} \text { (syst.) } \\
& \left.A_{\mathrm{DN}}(\mathrm{NC})=0.204 \pm 0.169 \text { (stat. }\right)_{-0.024}^{+0.025} \text { (syst.). }
\end{aligned}
$$

As is the case for the rates, there is also redundancy in the asymmetry measurements,

$$
A_{\mathrm{DN}}(\mathrm{ES})=\frac{A_{\mathrm{DN}}(\mathrm{CC})}{1+\frac{\epsilon R\left({ }^{8} \mathrm{~B} ; \mathrm{NC}\right)}{(1-\epsilon) R\left({ }^{8} \mathrm{~B} ; \mathrm{CC}\right)}}+\frac{A_{\mathrm{DN}}(\mathrm{NC})}{1+\frac{(1-\epsilon) R\left({ }^{8} \mathrm{~B} ; \mathrm{CC}\right)}{\epsilon R\left({ }^{8} \mathrm{~B} ; \mathrm{NC}\right)}}
$$

From the measurements (145) we find independently of the solar model, $R\left({ }^{8} \mathrm{~B} ; \mathrm{CC}\right) / R\left({ }^{8} \mathrm{~B} ; \mathrm{NC}\right)=$ $0.346_{-0.048}^{+0.046}$, and with that from Eq. (149) the central value, $A_{\mathrm{DN}}(\mathrm{ES})=-0.021$, in agreement with the result (147) and the first result (148), but the uncertainties are large and strongly anti-correlated [363] between $A_{\mathrm{DN}}(\mathrm{CC})$ and $A_{\mathrm{DN}}(\mathrm{NC})$. If one assumes that flavor oscillations into active neutrinos saturate the solar flux, $R\left({ }^{8} \mathrm{~B} ; \mathrm{NC}\right)=1$ and $A_{\mathrm{DN}}(\mathrm{NC})=0$, then there is only one independent asymmetry and with these constraints SNO finds 363,

$$
\left.A_{\mathrm{DN}}(\mathrm{CC})=-0.070 \pm 0.049 \text { (stat.) }\right)_{-0.013}^{+0.012} \text { (syst.). }
$$

This agrees with the Super-Kamiokande result obtained from (147) and Eq. (149),

$$
\left.A_{\mathrm{DN}}(\mathrm{CC})=-0.032 \pm 0.031 \text { (stat.) }\right)_{-0.018}^{+0.020} \text { (syst.), }
$$

and can be combined with the SNO result to give,

$$
A_{\mathrm{DN}}(\mathrm{CC})=-0.045_{-0.029}^{+0.030}
$$

which is non-vanishing at the $1.5 \sigma \mathrm{CL}$.

Super-Kamiokande and SNO have enough statistics to divide their data simultaneously into energy and zenith angle (day-night in the case of SNO) bins. The observed energy dependence is in both cases consistent with SSM expectations.

\subsubsection{The large mixing angle MSW solution to the solar neutrino problem}

In the past, there have been several solutions of the solar neutrino deficit problem in terms of neutrino oscillations depending on the mass splitting, $\Delta m_{\odot}^{2}$, and the mixing angle, $\theta_{\odot}$. The quality of the best fit within each solution has varied significantly over time. All but one of these solutions are by now highly disfavored. For example, the absence of spectral distortions as mentioned in the previous paragraph rules out any solution at small mixing 353] independently of solar model flux predictions. The same conclusion applies 353] to the vacuum oscillation solution characterized by very small mass splittings. Some other parameter regions predict large day-night variations (by matter effects in the Earth) contradicting observations still independent of any solar model. The predictions of the SSM enter only at the level of rate comparisons which strongly favor a solution proposed by Mikheev, Smirnov [364], 


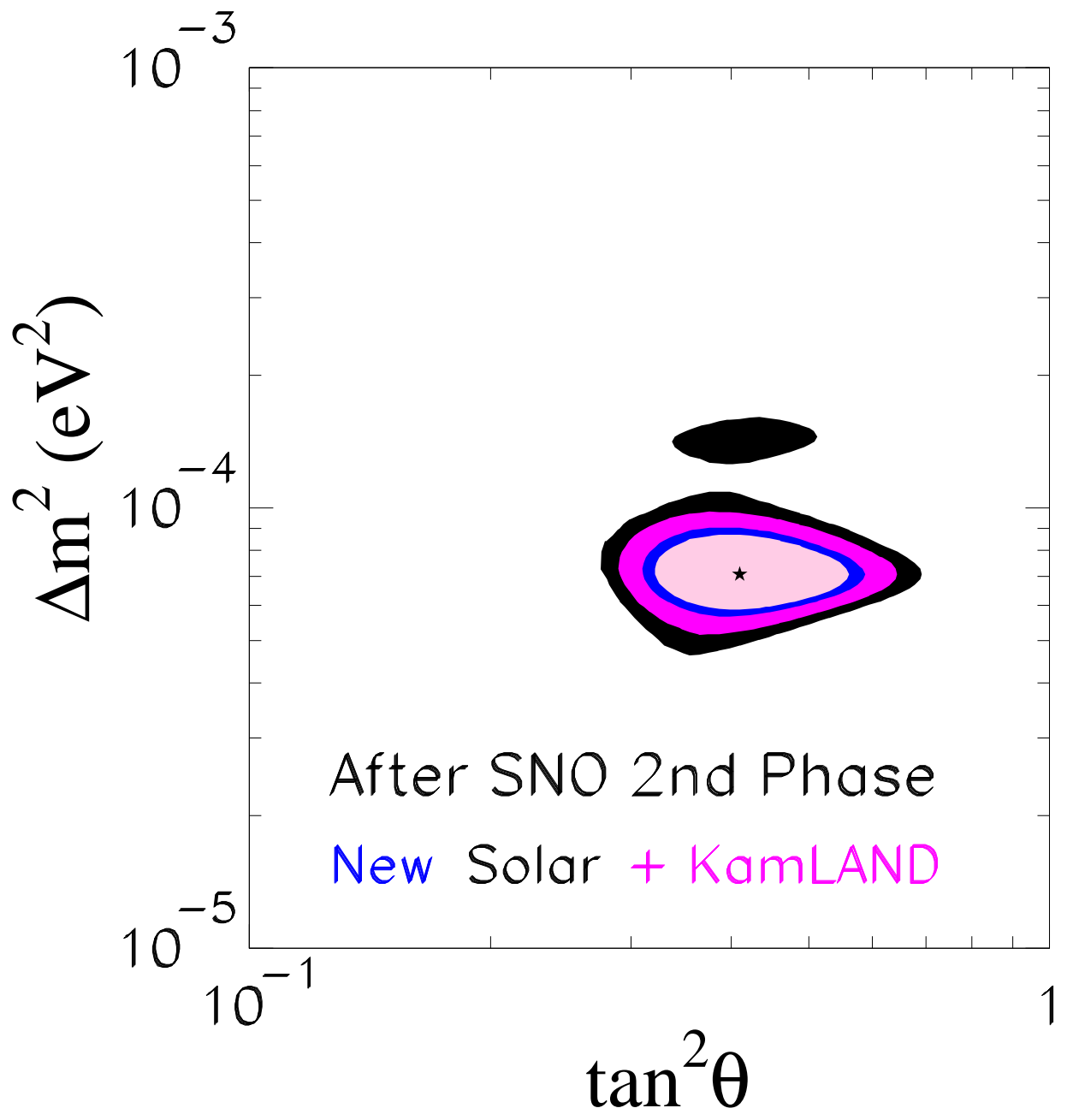

Figure 6: The allowed parameter space (from Ref. 374]) at 90\%, 95\%, 99\%, and 99.73\% CL in the $\tan ^{2} \theta_{\odot}-\Delta m_{\odot}^{2}$ plane. Note that without loss of generality one can take $\Delta m_{\odot}^{2}$ nonnegative. $\theta_{\odot}$ is then defined in the interval $[0, \pi / 2] . \theta_{\odot}>\pi / 4$ is allowed by the KamLAND reactor neutrinos since Eq. (127) (valid in vacuum) is symmetric under $\theta \rightarrow \pi / 2-\theta$, but $\theta_{\odot}<\pi / 4$ is selected by the requirement to have an MSW resonance effect.

and Wolfenstein 365. (MSW). MSW type solutions invoke a resonant enhancement (for $\theta_{\odot}>\pi / 4$ one predicts a reduction) of flavor oscillations by matter effects in the sun and can occur for a variety of parameters. But only the MSW solution with a large mixing angle and relatively large $\Delta m_{\odot}^{2}$ gives a good description of all data. Moreover, this solution has been confirmed by the KamLAND reactor experiment as discussed in Section [5.5. Combining the KamLAND and all solar neutrino experiments [366 374] splits the large angle MSW solution region into two parts ${ }^{18}$ as shown in Figure 6 ,

Since the MSW effect is based on neutrino interactions in the sun it can also (in parts of parameter space) distinguish between oscillations into active vs. sterile neutrinos. The large mixing angle solution fits the data only for oscillations dominantly into active neutrinos, in perfect agreement with the NC cross section seen by SNO.

\footnotetext{
${ }^{18}$ After this Section had been completed, the $\mathrm{NaCl}$ phase data of SNO 375] (see Section [5.2.1) and some new results by the SAGE and GNO Collaborations appeared. These are included in Figure [6] and have the effect of almost eliminating the upper part of the allowed region which now appears only at the 99.73\% CL.
} 


\subsection{Atmospheric Neutrinos}

Cosmic rays hitting the upper atmosphere of the Earth produce a rich source of neutrinos with variation in flavor (primarily $\nu_{e}, \bar{\nu}_{e}, \nu_{\mu}$, and $\bar{\nu}_{\mu}$ ), energy (experiments probe the range from $\mathcal{O}(100 \mathrm{MeV})$ to $\mathcal{O}(100 \mathrm{GeV})$, and distance (depending on the zenith angle $L$ ranges from $\mathcal{O}(10 \mathrm{~km})$ to the Earth radius of about $13,000 \mathrm{~km}$ ). Neutrinos with energies of no more than a few $\mathrm{GeV}$ produce events that are fully contained (FC) in the detectors. Muons produced by neutrinos outside the detector can also be studied if they are energetic enough to enter the detector and if they are going up so that they can be distinguished from cosmic ray muons. If such muons have energies of $\mathcal{O}(10 \mathrm{GeV})$ they are likely to stop within the detector and are referred to as partially contained (PC). If they have energies even larger than this they go through the detector.

\subsubsection{Rates}

The Kamiokande-II Collaboration was the first to observe [376] that the ratio ${ }^{19}, R_{\mu e}$, of muon neutrinos, $\nu_{\mu}$ or $\bar{\nu}_{\mu}$, to electron neutrinos, $\nu_{e}$ or $\bar{\nu}_{e}$, is smaller than the expected value of $R_{\mu e} \approx 2$. Kamiokande determines [377] the double ratio of observed to expected events (which from now on we will take as the definition of $R_{\mu e}$ ),

$$
R_{\mu e}=0.60_{-0.05}^{+0.06} \text { (stat.) } \pm 0.05 \text { (syst.), } \quad R_{\mu e}=0.57_{-0.07}^{+0.08} \text { (stat.) } \pm 0.07 \text { (syst.), }
$$

where the first (second) value is for $\nu$ s with an average energy in the sub-GeV (multi-GeV) region. These correspond to $3.9 \sigma$ and $2.3 \sigma$ deficits, respectively. A similar deficit was seen 378 with the IMB-3 Cherenkov detector in the Morton salt mine in Ohio, but it was not observed [379] with iron calorimeters such as the detector in the Frejus Underground Laboratory close to the French-Italian border. On the other hand, the 3.3 times larger Super-Kamiokande detector confirmed [380 381] the results (153),

$$
R_{\mu e}=0.61 \pm 0.03 \text { (stat.) } \pm 0.05 \text { (syst.), } \quad R_{\mu e}=0.66 \pm 0.06 \text { (stat.) } \pm 0.08 \text { (syst.). }
$$

Subsequently, the Soudan-2 Collaboration in the Tower-Soudan Iron Mine in Minnesota reported [382] the precise measurement for sub-GeV neutrinos,

$$
R_{\mu e}=0.64 \pm 0.11 \text { (stat.) } \pm 0.06 \text { (syst.). }
$$

This is only a $2.3 \sigma$ effect but it is in perfect agreement with (153) and (154) and the first time that an iron calorimeter confirmed the atmospheric neutrino deficit seen with water Čherenkov detectors.

We combine the results (153), (154), and (155) assuming that the smaller systematic uncertainty between Kamiokande and Super-Kamiokande is common to both, and that Soudan-2 is uncorrelated with the water Čherenkov results. The results,

$$
R_{\mu e}=0.614_{-0.051}^{+0.052}, \quad R_{\mu e}=0.63 \pm 0.09,
$$

correspond to $5.8 \sigma$ and $3.2 \sigma$ deficits, respectively.

\subsubsection{Zenith angle and spectral distributions}

Super-Kamiokande 381] also formed the ratio of the number of upward to downward $\mu$-like events,

$$
R^{\uparrow \downarrow}=0.52_{-0.06}^{+0.07} \text { (stat.) } \pm 0.01 \text { (syst.) } \quad \text { [expected: } 0.98 \pm 0.03 \text { (stat.) } \pm 0.02 \text { (syst.)] }
$$

\footnotetext{
${ }^{19}$ There are also several experiments quoting observed to expected $\nu_{\mu}$ flux ratios, but the uncertainty from the flux prediction is much larger than in $R_{\mu e}$. Nevertheless, most of these experiments are consistent with the atmospheric neutrino deficit.
} 
This means a $4.5 \sigma$ effect in a ratio that is independent of the overall flux prediction, while the analogous ratio for $e$-like events was consistent with unity. The significance in both ratios, $R_{\mu e}$ and $R^{\uparrow \downarrow}$, increased further and in Ref. 383 Super-Kamiokande provided detailed distributions of the event rates vs. zenith angle and $L / E_{\nu}$, demonstrating an excellent fit for two-flavor oscillations into $\nu_{\tau}$ or $\nu_{s}$ with $\sin ^{2} 2 \theta>0.82$ and $0.0005 \mathrm{eV}^{2}<\Delta m^{2}<0.006 \mathrm{eV}^{2}$ at $90 \% \mathrm{CL}$. Agreement of the $e$-like data with expectations disfavored oscillations into $\nu_{e}$ in agreement with the exclusion regions established by reactor experiments as discussed in Section 5.5. Furthermore, a study of upward through-going muons of minimum energy $1.6 \mathrm{GeV}$ by Super-Kamiokande found that the absolute flux is in agreement with the prediction 384 while the zenith angle dependence does not agree with no-oscillation predictions. Rather, the observed distortion suggested $\nu_{\mu} \rightarrow \nu_{\tau}$ or $\nu_{\mu} \rightarrow \nu_{s}$ oscillations and so did the double ratio of observed to expected PC neutrinos relative to through-going ones [385],

$$
0.22 \pm 0.02 \text { (stat.) } \pm 0.01 \text { (syst.) } \quad \text { [expected: } 0.37_{-0.04}^{+0.05} \text { (theo.)] }
$$

which revealed another $3.5 \sigma$ deficit. A simultaneous fit to zenith angle distributions of upward stopping and through-going muons yielded, $\sin ^{2} 2 \theta>0.7$ and $0.0015 \mathrm{eV}^{2}<\Delta m^{2}<0.015 \mathrm{eV}^{2}$ at $90 \% \mathrm{CL}$ in agreement with the FC sample. Finally, the difference in zenith angle distribution due to NCs and matter effects rejected the hypothesis of oscillations into sterile neutrinos at the 99\% CL, while $\nu_{\mu} \rightarrow \nu_{\tau}$ oscillations are sufficient to explain all results [386].

The MACRO detector at Gran Sasso confirmed [387] the preference for $\nu_{\mu} \rightarrow \nu_{\tau}$ oscillations of atmospheric neutrinos using their through-going muon sample. They also studied [388 the spectral and angular dependence of through-going muons, and provide a measurement of the ratio of vertical $(\cos \theta \leq-0.7)$ to horizontal $(\cos \theta \leq-0.7)$ events,

$$
1.48 \pm 0.13 \quad \text { [expected: } 2.20 \pm 0.17]
$$

which differs by $3.4 \sigma$ from the no-oscillation hypothesis while it is in reasonable agreement with the oscillation prediction, $1.70 \pm 0.14$.

The latest piece of evidence for atmospheric neutrino oscillations came from the K2K accelerator neutrino experiments as discussed in Section 5.4. Ref. 389. presents a simultaneous analysis of the Super-Kamiokande and K2K results and quote,

$$
\sin ^{2} 2 \theta=1.00_{-0.05}^{+0.00}, \quad \Delta m^{2}=0.0026 \pm 0.0004 \mathrm{eV}^{2}
$$

\subsection{Accelerator Neutrinos}

As can be seen from Eq. (127), an ideal experiment searching for two-neutrino oscillations is characterized by only two parameters, $P\left(\nu_{\alpha} \rightarrow \nu_{\beta}\right)$ and $L / E$. The oscillation probability for a realistic experiment in which there is some uncertainty or variation in $P, L$, and/or $E$ can be much more complicated (and may be based on an event-by-event likelihood), but the resulting limits are often still characterized by only two parameters although these may not describe every detail of an exclusion region [390]. This is frequently the case for accelerator and reactor neutrino experiments with known neutrino fluxes, and with a single detector whose size is small compared to $L$. In such cases $P\left(\nu_{\alpha} \rightarrow \nu_{\beta}\right)$ and $L / E$ (or effective definitions thereof) translate into a lower limit on $\sin ^{2} 2 \theta$ at large $\Delta m^{2}$ independent of $L / E$, and a lower limit on $\Delta m^{2}$ at maximal mixing, $\sin ^{2} 2 \theta=1$.

The KARMEN Collaboration searched for neutrino oscillations at the ISIS Neutron Spallation Source at the Rutherford Appleton Laboratory in England studying $\mu^{+}$decays at rest. Their oscillation length was $L=17.6 \mathrm{~m}$, and the neutrino energy at their kinematic endpoint was $E=52.8 \mathrm{MeV}$. In the $\bar{\nu}_{\mu} \rightarrow \bar{\nu}_{e}$ appearance mode, studied in inverse $\beta$ decay, $\bar{\nu}_{e} p \rightarrow e^{+} n, 15$ candidate reactions were seen 
with the KARMEN 2 detector (operating from February 1997 through March 2000) which is consistent with $15.8 \pm 0.5$ expected background events. The Collaboration sets the $90 \%$ CL limits [391],

$$
\begin{array}{ll}
\sin ^{2} 2 \theta<0.0017 & \left(\Delta m^{2}>100 \mathrm{eV}^{2}\right) \\
\Delta m^{2}<0.055 \mathrm{eV}^{2} & \left(\sin ^{2} 2 \theta=1\right) .
\end{array}
$$

This excludes most of the parameter space to which the LSND Collaboration at the Los Alamos Neutron Science Center had access to in a similar experiment, but with $L$ dominantly at $110 \mathrm{~m}$ and $135 \mathrm{~m}$ (1993-1995) and at $L=30 \mathrm{~m}$ (1996-1998). In contrast to KARMEN, LSND reports [392] a signal of $87.9 \pm 22.4 \pm 6.0$ inverse $\beta$ decay events above the expected background. This excess corresponds to an oscillation probability of $P=0.264 \pm 0.067 \pm 0.045$ (a $3.3 \sigma$ effect), and a best fit occurring at,

$$
\sin ^{2} 2 \theta=0.003, \quad \Delta m^{2}=1.2 \mathrm{eV}^{2} .
$$

This is excluded by the limits (161), but its larger values of $L$ extends the sensitivity of LSND to slightly smaller values of $\Delta m^{2}$ so that the findings of the two groups do not necessarily conflict. The BooNE Collaboration [393] at FNAL is able to test the LSND result in both the $\nu_{\mu} \rightarrow \nu_{e}$ appearance and the $\nu_{\mu}$ disappearance modes. Data taking of the single detector version of the experiment, MiniBooNE, commenced in August 2002. If a positive signal is seen, the experiment will be upgraded by a second detector at a distance optimized for a precision determination of oscillation parameters and searches for $\mathrm{CP}$ and $\mathrm{CPT}$ violation ${ }^{20}$ in the lepton sector.

The results of the reactor disappearance experiments discussed in Subsection [5.5] can be used as constraints on $\nu_{e} \rightarrow \nu_{\tau}$ oscillations. In addition, there were two $\nu_{\tau}$ appearance experiments, CHORUS [396] and NOMAD [397], at the SPS accelerator at CERN operating from 1994 through 1997. The $450 \mathrm{GeV}$ protons of the SPS were aimed at a Beryllium target located $850 \mathrm{~m}(940 \mathrm{~m})$ from the CHORUS (NOMAD) detector. The average values of $L$ for the two experiments were a few hundred meters shorter than this. The average neutrino energy of the almost pure $\nu_{\mu}$ wide-band beam was $E \approx 25 \mathrm{GeV}$. Due to the relatively small $L / E$, these experiments were only sensitive to larger $\Delta m^{2}$, but the small $\nu_{e}$ component of the beam was sufficient to achieve stronger limits on $\nu_{e}-\nu_{\tau}$ mixing at large mass splittings than the reactor experiments discussed in Subsection [5.5.

$$
\begin{array}{ll}
\sin ^{2} 2 \theta<0.052 & \text { (CHORUS at large } \Delta m^{2} \text { ), } \\
\sin ^{2} 2 \theta<0.015 & \text { (NOMAD at large } \Delta m^{2} \text {. }
\end{array}
$$

The primary physics goal of CHORUS and NOMAD, however, were studies of $\nu_{\mu} \rightarrow \nu_{\tau}$ oscillations. CHORUS set limits,

$$
\begin{array}{ll}
\sin ^{2} 2 \theta<0.00068 & \left(\text { large } \Delta m^{2}\right), \\
\Delta m^{2}<0.6 \mathrm{eV}^{2} & \left(\sin ^{2} 2 \theta=1\right),
\end{array}
$$

while NOMAD finds,

$$
\begin{array}{ll}
\sin ^{2} 2 \theta<0.00033 & \left(\text { large } \Delta m^{2}\right), \\
\Delta m^{2}<0.7 \mathrm{eV}^{2} & \left(\sin ^{2} 2 \theta=1\right) .
\end{array}
$$

These limits are complemented at large mixing by an older (1983) $\nu_{\mu}$ disappearance experiment 398. at the PS accelerator at CERN. The Collaboration (which continued later as CDHSW Collaboration) took data at distances, $L=130 \mathrm{~m}$ and $885 \mathrm{~m}$ from the target and excluded,

$$
0.23 \mathrm{eV}^{2}<\Delta m^{2}<100 \mathrm{eV}^{2} \quad\left(\sin ^{2} 2 \theta=1\right)
$$

\footnotetext{
${ }^{20}$ If the LSND signal can be confirmed, the most likely interpretation of it will not be in terms of a forth neutrino but rather in terms of a more fundamental type of NP such as CPT violation [394 since any additional neutrino is strongly constrained [395] by big bang nucleosynthesis. This is true even for sterile neutrinos as long as there is appreciable mixing with active neutrinos as would apply to LSND.
} 
Thus, the lower limit was not superseded by the corresponding ones from CHORUS and NOMAD. It is a limit of considerable interest since the atmospheric neutrino analysis of the Super-Kamiokande Collaboration implies large $\nu_{\mu}-\nu_{\tau}$ mixing. However, even the limit (166) is still about two orders of magnitude above the $\Delta m^{2}$ values preferred by Super-Kamiokande.

An accelerator neutrino disappearance experiment, $\mathrm{K} 2 \mathrm{~K}$, with a long enough $(L=250 \mathrm{~km})$ baseline to test atmospheric neutrino oscillations has been designed using a $12 \mathrm{GeV}$ proton beam from the KEK proton synchrotron. The produced neutrino beam consists to $98 \%$ of $\nu_{\mu}$ with an average energy of 1.3 $\mathrm{GeV}$. The $\nu_{\mu}$ beam is monitored by a near detector $300 \mathrm{~m}$ from the target. Located at the far end is the Super-Kamiokande detector. A reduction of the $\nu_{\mu}$ flux (56 events were observed and 80.1 $1_{-5.4}^{+6.2}$ expected) together with a distortion of the energy spectrum indicate neutrino oscillations 399] at the 99\% CL. The oscillation parameters are in perfect agreement with the results implied by the atmospheric neutrinos.

A similar $\nu_{\mu}$ disappearance experiment, MINOS [400], is planned using the NuMI beam currently under construction at FNAL. NuMI will be produced with the $120 \mathrm{GeV}$ proton beam of the Main Injector striking a movable carbon target which will allow a variation in the average neutrino energy between about 3 and $15 \mathrm{GeV}$. The $731 \mathrm{~km}$ baseline will pass through a near detector at FNAL and a similar detector in the Soudan mine. MINOS will provide large statistics and controlled systematics enabling the Collaboration to address the atmospheric neutrino oscillation hypothesis in both CC and NC channels. Limits (or measurements) on $\nu_{\mu} \rightarrow \nu_{e}$ and $\nu_{\mu} \rightarrow \nu_{s}$ oscillations are also physics goals.

Two experiments at Gran Sasso [401, Opera and Icarus, plan to prove the $\nu_{\mu} \rightarrow \nu_{\tau}$ oscillation hypothesis by directly detecting the $\nu_{\tau}$. They will have virtually identical baselines compared to MINOS $(L=732 \mathrm{~km})$ and will be using the future CERN high energy $\nu_{\mu}$ beam, CNGS. In addition, they will also be able to test a possible subleading $\nu_{\mu} \rightarrow \nu_{e}$ transition in $\nu_{e}$ appearance mode. Completion of CNGS and begin of data taking is scheduled for 2006.

\subsection{Reactor Neutrinos}

Neutrinos produced at nuclear power plants have been studied in $\bar{\nu}_{e}$ disappearance experiments. The reaction in which $\bar{\nu}_{e}$ oscillate into $\bar{\nu}_{\mu}$ is the CP conjugate relative to the KARMEN and LSND accelerator experiments discussed in Subsection 5.4 and are therefore complementary. The reactor experiments in France and Arizona are short-baseline experiments, while KamLAND with two orders of magnitude longer baselines is motivated by and sensitive to the oscillation parameters suggested by the LMA solution to the solar neutrino problem.

A high statistics measurement [402] of neutrino energy spectra was carried out at $L=15,40$ and $95 \mathrm{~m}$ from the Bugey reactor in France. No oscillations were reported, and the limits,

$$
\begin{array}{ll}
\sin ^{2} 2 \theta<0.02 & \left(\text { large } \Delta m^{2}\right), \\
\Delta m^{2}<0.01 \mathrm{eV}^{2} & \left(\sin ^{2} 2 \theta=1\right),
\end{array}
$$

could be set, limiting the unexcluded LSND parameter space to small mixing.

In comparison to the Bugey experiment, the Chooz and Palo Verde experiments had sensitivity to smaller $\Delta m^{2}$ but not as small mixing. The experiment near Chooz in France studied neutrinos with $E \sim 3 \mathrm{MeV}$ that originated from two reactors about $1 \mathrm{~km}$ away. The 200 day exposure included substantial periods with only one or the other reactor operating, in addition to about 143 days of background studies with both reactors turned off. The results [03],

$$
\begin{array}{ll}
\sin ^{2} 2 \theta<0.10 & \left(\text { large } \Delta m^{2}\right), \\
\Delta m^{2}<0.0007 \mathrm{eV}^{2} & \left(\sin ^{2} 2 \theta=1\right),
\end{array}
$$

excluded $\Delta m^{2}$ values at large mixing almost down to the LMA solution of the solar neutrino problem. The Collaboration also presented results of lower sensitivity (due to statistical limitations) but independent of the absolute normalization of the $\bar{\nu}_{e}$ flux, the cross section, the number of target protons, 
and detector efficiencies. They were based only on the comparison of the positron spectra from the two nuclear reactors located at different distances, $\Delta L=116.7 \mathrm{~m}$.

An experiment 404] with similar reach took data between September 1998 and July 2000 near the Palo Verde Nuclear Generating Station in Arizona. A detector was exposed to the reactor for 350 days at distances $L=750$ and $890 \mathrm{~m}$. This included 108 days with one of the three reactors turned off, which could be used for additional background studies. Non-observation of neutrino oscillations in the $\bar{\nu}_{e}$ disappearance mode resulted in the $90 \%$ CL limits,

$$
\begin{array}{ll}
\sin ^{2} 2 \theta<0.17 & \left(\text { large } \Delta m^{2}\right), \\
\Delta m^{2}<0.0011 \mathrm{eV}^{2} & \left(\sin ^{2} 2 \theta=1\right) .
\end{array}
$$

None of the limits of these three reactor experiments probes values of $\Delta m^{2}$ small enough to address the solar neutrino problem. For this one needs to extend the baseline, $L$, by about two orders of magnitude. This is the purpose of KamLAND, the largest low energy anti-neutrino detector, located in the Kamioka mine. The $\bar{\nu}_{e}$ flux contains energies up to about $3.5 \mathrm{MeV}$ and originates from a variety of reactors with an average $L \approx 180 \mathrm{~km}$. The dominant fraction of $79 \%$ of the flux is produced by reactors with distances between 138 and $214 \mathrm{~km}$ from KamLAND. 6.7\% of the flux is due to a reactor at $L=88 \mathrm{~km}$, with the rest traveling $L \geq 295 \mathrm{~km}$. Very recently, the KamLAND Collaboration presented results of their first 145.1 days of data taking. The number of observed neutrinos (reduced by the expected background) divided by the number of expected signal events assuming no oscillations is quoted as [05],

$$
\frac{N_{\text {obs }}}{N_{\text {expected }}}=0.611 \pm 0.085 \text { (stat.) } \pm 0.041 \text { (syst.). }
$$

This is a $4.1 \sigma$ deficit, strongly suggestive of $\bar{\nu}_{e}$ disappearance, and therefore the first reactor indication for neutrino oscillations. Moreover, the KamLAND Collaboration used spectral shape information to perform a fit to two-neutrino oscillation parameters, yielding $\sin ^{2} 2 \theta=1.01$ and $\Delta m^{2}=6.9 \times 10^{-5} \mathrm{eV}^{2}$ at the minimum of the $\chi^{2}$ function. These values are in good agreement with the LMA solution of the solar neutrino problem.

\subsection{Neutrinoless Double $\beta$ Decay}

A fundamental question left open by the neutrino oscillation experiments is whether the neutrino is a Dirac or Majorana particle. As discussed above, in most scenarios that generate a massive neutrino, such as the see-saw mechanism [248 250], the neutrino has a Majorana mass term. Perhaps the most direct test of this possibility is carried out through the search for a neutrinoless final state in the double $\beta$ decay of heavy $0^{+}$nuclei,

$$
A(Z, N) \rightarrow A(Z \mp 2, N \pm 2)+2 e^{ \pm}
$$

Since these modes entail a change of total lepton number (L) by two units, a non-zero result would imply that the neutrino is its own antiparticle. Experimentally, the signature for the $0 \nu$ mode is characterized by a sharp peak at the endpoint of the $2 \nu \beta \beta$ decay spectrum. Until recently, no statistically significant $0 \nu$ peak had been observed, and this absence led to upper bounds on the rate for neutrinoless decay.

A summary of representative, present limits on $T_{1 / 2}^{0 \nu}$ from a variety of experiments is given in Table 10. The most stringent limits had been obtained from two experiments using Ge detectors, the HeidelbergMoscow (HM) 407408 and IGEX experiments 409410. The two experiments employed comparable quantities of $86 \%$ enriched ${ }^{76} \mathrm{Ge}$ and quoted similar background levels of 0.20 counts $/(\mathrm{keV} \cdot \mathrm{kg} \cdot \mathrm{yr})$. However, a different limiting factor in background suppression was identified in the two experiments. The HM Collaboration saw the presence of copper in the cryostat as the dominant limitation, whereas in the IGEX experiment, the presence of radon and activated ${ }^{68} \mathrm{Ge}$ were considered the most serious backgrounds. The $T_{1 / 2}^{0 \nu}$ limits for the two experiments are comparable. 
Table 10: Representative present (upper part) and prospective (lower part) $0 \nu \beta \beta$ limits, compiled from Refs. 320 406. The forth column shows a range of theoretical expectations for $T_{1 / 2}^{0 \nu \beta \beta}$ for $\left\langle m_{\nu}\right\rangle=50 \mathrm{eV}$ given various nuclear matrix element computations. The limits on $\left\langle m_{\nu}\right\rangle$ in the last colmun of the upper part of the Table are those quoted in the experimental papers; the ranges in the lower part indicate proposed sensitivities.

\begin{tabular}{|c|l|r|r|r|}
\hline Isotope & Experiment & $T_{1 / 2}^{0 \nu}(y)[90 \% \mathrm{CL}]$ & $T_{1 / 2}^{0 \nu}(y)[$ Theory] & $\left\langle m_{\nu}\right\rangle[\mathrm{eV}]$ \\
\hline${ }^{76} \mathrm{Ge}$ & HM [407408] & $1.9 \times 10^{25}$ & $6.8-70.8 \times 10^{26}$ & $<0.35$ \\
${ }^{76} \mathrm{Ge}$ & IGEX [409410] & $1.6 \times 10^{25}$ & $6.8-70.8 \times 10^{26}$ & $<0.33-1.25$ \\
${ }^{136} \mathrm{Xe}$ & Gotthard 411] & $4.4 \times 10^{23}$ & $7.2-48.4 \times 10^{26}$ & $<1.8-5.2$ \\
${ }^{130} \mathrm{Te}$ & MIBETA [412] & $1.4 \times 10^{23}$ & $0.6-23.2 \times 10^{26}$ & $<1.1-2.6$ \\
${ }^{100} \mathrm{Mo}$ & ELEGANTS [413] & $5.5 \times 10^{22}$ & $1.2-15.6 \times 10^{26}$ & $<2.1$ \\
\hline${ }^{76} \mathrm{Ge}$ & GENIUS 414] & $1 \times 10^{28}$ & $6.8-70.8 \times 10^{26}$ & $0.013-0.042$ \\
${ }^{76} \mathrm{Ge}$ & Majorana [415] & $3 \times 10^{27}$ & $6.8-70.8 \times 10^{26}$ & $0.024-0.077$ \\
${ }^{136} \mathrm{Xe}$ & EXO 416] & $8 \times 10^{26}$ & $7.2-48.4 \times 10^{26}$ & $0.050-0.120$ \\
${ }^{130} \mathrm{Te}$ & CUORE [417] & $2 \times 10^{26}$ & $0.6-23.2 \times 10^{26}$ & $0.050-0.170$ \\
${ }^{100} \mathrm{Mo}$ & MOON [418] & $1 \times 10^{27}$ & $1.2-15.6 \times 10^{26}$ & $0.017-0.060$ \\
\hline
\end{tabular}

Recently, a subset of the HM Collaboration has reported a signal for neutrinoless decay. While the initial report [419] generated controversy 420, an analysis of additional data indicates a $4 \sigma$ effect, with $T_{1 / 2}^{0 \nu}=(0.69 \rightarrow 4.18) \times 10^{25}$ y at $99.73 \% \mathrm{CL}$ [421]. Using an estimate for the uncertainty in nuclear matrix elements (see below), the authors derive a range for the effective mass of the exchanged Majorana neutrino $-\left\langle m_{\nu}\right\rangle=(0.1 \rightarrow 0.9) \mathrm{eV}$ - that would indicate a highly degenerate neutrino spectrum. Given the potential implications of this result, independent experimental confirmation (e.g., using a different nucleus and experimental method) is clearly warranted.

A number of proposals have been developed for improving the rate sensitivity by several orders of magnitude. These proposals are discussed extensively in the recent review by Elliot and Vogel [406], and we do not repeat their discussion here. However, in Table 10, we summarize the goals of the five most widely discussed proposals: GENIUS [414] (GErmanium NItrogen Underground Setup) that would consist of an array of $2.5 \mathrm{~kg}$ Ge crystals; Majorana 415] that would build a $500 \mathrm{~kg}$ detector from 210 segmented Ge crystals; EXO [416] (Enriched Xenon Observatory) that proposes to employ 10 tons of $60-80 \%$ enriched ${ }^{136}$ Xe in the Waste Isolation Pilot Plant (WIPP); CUORE [417] (Cryogenic Underground Observatory for Rare Events) at Gran Sasso that would use $1000 \mathrm{TeO}_{2}$ crystals; and MOON (MOlybdenum Observatory of Neutrinos) that would use 34 tons of natural Mo. The designs of the two Ge experiments follow from the differently identified limiting backgrounds in the HM and IGEX experiments, respectively. The segmentation of the crystals in the Majorana detector would permit veto of ${ }^{68} \mathrm{Ge}$ induced events that were highlighted in the IGEX experiment. In contrast, the Ge crystals in the GENIUS experiment would be isolated in a liquid nitrogen bath from the external sources of radioactivity considered most problematic by the HM Collaboration.

From a theoretical standpoint, the reliable extraction of limits on neutrino properties from the $T_{1 / 2}^{0 \nu}$ lower bounds have proved as challenging as the experiments themselves. The most common theoretical analyses assume that neutrinoless decay is mediated by the exchange of a light Majorana neutrino. In this case, the rate is proportional to the square of the effective mass,

$$
\left\langle m_{\nu}\right\rangle=\left.\left|\sum_{j}\right| U_{e j}\right|^{2} e^{i \alpha_{j}} m_{j} \mid,
$$


where the sum runs over all light neutrino generations, the $U_{e j}$ connects left handed electron neutrino weak eigenstate to the Majorana mass eigenstates labeled by the index, $j$, and the $\alpha_{j}$ are the $N-1$ Majorana phases in the general neutrino mass matrix. Note that the presence of these phases allows for the possibility of cancellations in the sum over light mass eigenstates.

The remaining theoretical input to the rate involves nuclear matrix elements. For the $0^{+} \rightarrow 0^{+}$ decays of interest here, one has,

$$
\frac{1}{T_{1 / 2}^{0 \nu}}=G^{0 \nu}\left(E_{0}, Z\right)\left|M_{G T}^{0 \nu}-\frac{g_{V}^{2}}{g_{A}^{2}} M_{F}^{0 \nu}\right|^{2}\left\langle m_{\nu}\right\rangle^{2},
$$

where $M_{F}^{0 \nu}$ and $M_{G T}^{0 \nu}$ are the Fermi and Gamow-Teller matrix elements for the $\Delta Q=2$ nuclear transition, respectively, $g_{V}\left(g_{A}\right)$ are the (axial) vector coupling of the $W$ boson to quarks, and $G^{0 \nu}\left(E_{0}, Z\right)$ is a calculable phase space factor that depends on the energy, $E_{0}$, released to the two electrons.

The matrix elements $M_{F, G T}^{0 \nu}$ depend on a joint neutrino-nucleus Green's function that involves a sum over all possible intermediate nuclear states. Nuclear model studies indicate that the sum is dominated by states within a fairly narrow range of energies and that it may be rather well approximated by using closure with an appropriate choice for the average excitation energy. Matrix elements of the resulting two-body operator must then be computed between the initial and final ground state wavefunctions. For the kinematics of the neutrinoless decay, the operator samples relatively high momentum components of the wave-functions that are not well constrained by existing measurements of nuclear properties. Different model calculations have led to a fairly broad spread in the values of the $M_{F, G T}^{0 \nu}$.

Two types of approaches have been taken [406, namely nuclear shell model computations and those involving the quasi-particle random phase approximation (QRPA). In the former case, the huge size of the single particle basis requires truncation of the model space to perform tractable computations. In order to account for omitted states, one generally employs effective operators that act within the truncated model space to compensate for the truncation. Since no one has, as yet, derived these effective operators by rigorously integrating out the omitted states, phenomenological tests of the effective operators must be employed to evaluate their degree of effectiveness. While such tests have proved successful in analyzing nuclear systematics such as magnetic moments or single particle Gamow-Teller transitions, there exist few, if any, independent tests of effective operators relevant to $M_{F, G T}^{0 \nu}$.

The QRPA approach entails performing a complete sum over particle-hole or particle-particle "bubbles". The strength of the particle-particle interaction, $g_{p p}$ can be adjusted to yield agreement with measured $2 \nu \beta \beta$ decay Gamow-Teller strengths and then used in calculations of $M_{F, G T}^{0 \nu}$. However, the value of $g_{p p}$ needed to account for $M_{G T}^{2 \nu}$ is close to the limit of validity for the QRPA equations, indicating the importance of more complicated configurations than the two particle or particle-hole configurations included in the QRPA framework. Were it not for the truncation problem, the shell model would provide a more realistic framework, since diagonalization of the shell model space includes the effect of complex configurations. As it stands, however, neither approach has proved completely satisfactory, and this situation is reflected in the wide variation in QPRA and shell model predictions for the $M_{F, G T}^{0 \nu}$. One rough guide to the theoretical uncertainty associated with these approximations is provided by a comparison of different calculations. In the case of ${ }^{76} \mathrm{Ge}$, for example, the spread in rates among various calculations is roughly an order of magnitude, leading to a factor of three spread in the values of $\left\langle m_{\nu}\right\rangle$. One should bear in mind, however, that such comparisons may overlook systematic shortcomings pertinent to all published calculations (such as the approximations employed in a given method) and, therefore, may underestimate the degree of theoretical uncertainty. Clearly, new ideas are needed for providing more robust computations of the $M_{F, G T}^{0 \nu}$ for the light Majorana neutrino scenario. These caveats notwithstanding, the proposed future $0 \nu \beta \beta$ decay experiments summarized above would improve the $\left\langle m_{\nu}\right\rangle$ sensitivity to the $50 \mathrm{meV}$ range.

The exchange of a light Majorana neutrino is not, however, the only mechanism by which the neutrinoless mode may occur. It is also possible that the exchange of a heavy particle drives the 
decay. In the case of the see-saw model [248 250], for example, cancellations in the sum (172) may suppress the effect of the light neutrinos, thereby exposing the effect of exchanged heavier Majorana neutrinos. Alternatively, the exchange of some other Majorana particle, such as the neutralino $\left(\chi^{0}\right)$ in SUSY scenarios may be dominant [22 423]. Recently, the framework for treating such heavy particle exchange in the nucleus has been placed on a firmer theoretical footing through the use of an EFT [424]. The EFT approach involves integrating out the heavy particle, and leaving dimension nine operators containing four quark and two lepton fields. In the most general case one has [424],

$$
\mathcal{L}_{0 \nu \beta \beta}=\frac{G_{F}^{2}}{\Lambda_{\beta \beta}} \sum_{j=1}^{14} C_{j}(\mu) \hat{O}_{j} \bar{e} \Gamma_{j} e^{c},
$$

where $\Lambda_{\beta \beta}$ is a mass scale associated with the heavy lepton number violating physics (e.g., the mass of the $\chi^{0}$ ), the $\hat{O}_{j}$ are four-quark operators, the $\Gamma_{j}$ are Dirac matrices, and the $C_{j}(\mu)$ are constants determined by a particular particle physics model. In order to compute the effects of $\mathcal{L}_{0 \nu \beta \beta}$ in $\Delta Q=2$ $0^{+} \rightarrow 0^{+}$transitions, one can construct the appropriate set of hadronic operators containing nucleon and pion fields that reflect the spacetime and chiral transformation properties of the various $\mathcal{O}_{j}$. The hadronic operators can be expanded systematically in powers of $Q / \Lambda_{\text {had }}$, where $Q$ is some momentum or mass relevant to nuclear processes and $\Lambda_{\text {had }}$ is the hadronic scale at which one matches the $\mathcal{O}_{j}$ onto the hadronic basis. One may then systematically construct an appropriate set of nuclear operators using this tower of effective hadronic interactions, and use them to compute ground state-to-ground state transition matrix elements.

The genesis for this development appeared in the work of Ref. [422], where the particular case of RPV SUSY was studied and where it was pointed out that long range, pion exchange nuclear operators could be generated by the relevant four-quark operators. A more comprehensive and systematic development of this framework using the ideas of EFTs was subsequently given in Ref. 424. In that work, additional scenarios for $0 \nu \beta \beta$ decay were discussed, and the importance of considering the spacetime and chiral properties of the relevant $\mathcal{O}_{j}$ illustrated. Under certain scenarios, these symmetries imply an enhancement of the nuclear matrix element via the existence of lower order operators that are otherwise forbidden. Additional theoretical work is needed, however, in order to fully implement these ideas. In particular, a refined treatment of QCD effects - such as the RG evolution of the $C_{j}(\mu)$ from the scale $\Lambda_{\beta \beta}$ down to $\Lambda_{\text {had }}$ - remains to be carried out. In addition, the degree to which the nuclear matrix elements reflect the systematic power counting in $Q / \Lambda_{\text {had }}$ of the hadronic and nuclear operators is an open question, as is the appropriate treatment of short range correlations in taking nuclear matrix elements. In this respect, new studies of parity violation in hadronic systems may provide useful guidance.

\subsection{Anomalous Magnetic Moment of the Muon}

The magnetic moment of the electron, $g_{e}$, provides the best determination of the fine structure constant, but is currently not measured precisely enough to give a sensitive probe of EW physics which is suppressed by a factor $(\alpha / \pi) m_{e}^{2} / M_{W}^{2} \sim \mathcal{O}\left(10^{-13}\right)$. Meaningful (but still weak) bounds on NP contributions can be set only if they enter without loop suppression. On the other hand, EW contributions to the anomalous magnetic moment of the muon ${ }^{21}$ [425], $a_{\mu}=\left(g_{\mu}-2\right) / 2$, are enhanced by a factor $m_{\mu}^{2} / m_{e}^{2} \sim 4 \times 10^{4}$, which renders them sizable enough to be detectable at the E821 experiment at the AGS at BNL.

Recently, the E821 Collaboration [426] published its final result, leading to the new world average,

$$
a_{\mu}(\text { exp. })-\frac{\alpha}{2 \pi}=(4511.07 \pm 0.63) \times 10^{-9} .
$$

\footnotetext{
${ }^{21} a_{\tau}$ has not yet been observed experimentally.
} 
Individual results on positively and negatively charged muons are in good agreement with each other and serve as a test of the CPT theorem. The value (175) is precise enough to provide a good laboratory to test the SM and probe theories beyond it [427]. For example, scenarios of low energy supersymmetry with large $\tan \beta$ and moderately light superparticle masses can give large contributions to $a_{\mu}$ [428].

Unfortunately, the interpretation of $a_{\mu}$ is compromised by large theoretical uncertainties introduced by hadronic effects. Two- and three-loop vacuum diagrams containing light quark loops ${ }^{22}$ cannot be calculated reliably in perturbative QCD (PQCD). Instead they are obtained by computing dispersion integrals over measured (at low energies) and theoretical (at higher energies) hadronic cross sections. At the two-loop level [430,

$$
a_{\mu}(\text { had; 2-loop })=\left(\frac{\alpha m_{\mu}}{3 \pi}\right)^{2} \int_{4 m_{\pi}^{2}}^{\infty} \frac{d s}{s^{2}} \hat{K}(s) R(s),
$$

where $R(s)$ is the cross section of $e^{+} e^{-} \rightarrow$ hadrons, normalized to the tree level cross section of $e^{+} e^{-} \rightarrow \mu^{+} \mu^{-}$, and where

$$
\hat{K}(s)=\int_{0}^{1} d x \frac{3 x^{2}(1-x)}{1-x+x^{2} m_{\mu}^{2} / s} .
$$

The uncertainty introduced by this procedure is comparable to the experimental one in (175). An analogous uncertainty occurs in the QED coupling constant, $\hat{\alpha}(\mu)$, preventing its precise theoretical computation from the fine structure constant, $\alpha$, for $\mu \gtrsim 2 m_{\pi^{0}}$. Note that knowledge of $\hat{\alpha}\left(M_{Z}\right)$ is indispensable for the extraction of $M_{H}$ from EW precision data (see Section 1.3). As a result, these hadronic uncertainties are strongly correlated with each other [429, with the renormalization group evolution of the weak mixing angle, and also with other fundamental SM parameters, such as the strong coupling constant and the heavy quark masses. In addition to $R(s)$, one can obtain additional experimental information using $\tau$ spectral functions and isospin symmetry when isospin breaking effects are properly taken into account. A $\tau$-based analysis yields [431,

$$
a_{\mu}-\frac{\alpha}{2 \pi}=(4509.83 \pm 0.58) \times 10^{-9}
$$

which is marginally (within $1.4 \sigma$ ) consistent with the result (175). On the other hand, using only $R(s)$ including the most recent data from the CMD-2 Collaboration [432] yields 431,

$$
a_{\mu}-\frac{\alpha}{2 \pi}=(4508.36 \pm 0.72) \times 10^{-9}
$$

i.e., a $2.8 \sigma$ deviation. The $R(s)$ driven value is also consistent with the findings of Refs. [433 434] which defined a higher cutoff for the transition from experimental data to PQCD. The difference between the evaluations (178) and (179) can be traced to a discrepancy between the $2 \pi$ spectral functions obtained from the two methods. For example, if one uses the $e^{+} e^{-}$data and CVC to predict the branching ratio for $\tau^{-} \rightarrow \nu_{\tau} \pi^{-} \pi^{0}$ decays one obtains $24.52 \pm 0.32 \%$ [431] while the average of the measured branching ratios by DELPHI [35., ALEPH, CLEO, L3, and OPAL 431] yields $25.43 \pm 0.09 \%$, which is $2.8 \sigma$ higher. It is important to understand the origin of this difference and to obtain additional experimental information (e.g., from the radiative return method [436]). Fortunately, this problem is less pronounced as far as $a_{\mu}^{\text {had }}$ is concerned: due to the suppression at large $s$ (from where the conflict originates) the difference is only $1.7 \sigma$ (or $1.9 \sigma$ if one adds the $4 \pi$ channel which by itself is consistent between the two methods). Note also that a part of this difference is due to the older $e^{+} e^{-}$data [431], and the direct conflict between $\tau$ decay data and CMD 2 is less significant. Isospin violating corrections have been estimated in Ref. [437] and found to be under control. The largest effect is due to higher order

\footnotetext{
${ }^{22}$ In contrast, the contributions from heavy quarks and from light quarks in the perturbative regime are known in analytic form 429.
} 
EW corrections [438] but introduces a negligible uncertainty 439]. If we view the $1.7 \sigma$ difference as a fluctuation and use all available information as constraints in a SM fit we find,

$$
a_{\mu}(\mathrm{SM})-\frac{\alpha}{2 \pi}=(4509.15 \pm 0.49) \times 10^{-9}
$$

which corresponds to a $2.4 \sigma$ deviations. Thus, regardless of whether one trusts the $\tau$-based analysis or follows Ref. [434] which argues that CVC breaking effects (e.g., through a relatively large mass difference between the $\rho^{ \pm}$and $\rho^{0}$ vector mesons) may be larger than expected ${ }^{23}$, one concludes in both cases that there is a deviation at the level of two and a half standard deviations.

The error in the prediction (180) is from the hadronic uncertainties excluding parametric ones such as from $\alpha_{s}$ and the heavy quark masses. We estimate its correlation with $\Delta \alpha\left(M_{Z}\right)$ to $-24 \%$. The uncertainty in (180) includes a contribution from the hadronic three-loop light-by-light scattering amplitude [440], $a_{\mu}(\mathrm{LBLS})=(+0.83 \pm 0.19) \times 10^{-9}$, which has been estimated within a form factor approach. Its sign is opposite relative to earlier work, and has subsequently been confirmed by two other groups 441442. A more rigorous calculation based on $\chi \mathrm{PT}$ confirmed the value of $a_{\mu}(\mathrm{LBLS})$ but could not exclude the possibility that its uncertainty might have been underestimated [443. In this context, $a_{\mu}$ (LBLS) depends on several a priori unknown constants, not all of which can be determined in a model-independent way from other measurements. The uncertainty in $a_{\mu}(\mathrm{LBLS})$ associated with the unknown constants could be substantially larger than what is quoted for the model calculation of Ref. [440, potentially reducing the significance of the deviation from the SM value (180). Other hadronic effects at three-loop order 444] contribute $a_{\mu}^{\text {had }}\left(\alpha^{3}\right)=(-1.00 \pm 0.06) \times 10^{-9}$.

Note added: After the completion of this Section, an update [445] appeared of the four-loop QED contribution reducing the discrepancy of $a_{\mu}$ with the SM by $0.22 \sigma$. There was also a new estimate 446 ] of $a_{\mu}(\mathrm{LBLS})=(+1.36 \pm 0.25) \times 10^{-9}$, which would reduce the discrepancy further by $2 / 3$ of a standard deviation.

\section{Weak Probes of the Strong Interaction}

\subsection{Hadronic Parity Violation}

Despite decades of experimental and theoretical study, achieving a solid understanding of the weak interaction between quarks remains an elusive goal. In contrast to the analysis of purely leptonic and semileptonic weak interactions, one cannot disentangle the effects of non-perturbative strong interactions between quarks from those generated by the weak interaction. This difficulty is reflected by a number of on-going puzzles in the $\Delta S=1$ sector, the best known being the $\Delta I=1 / 2$ rule. While the latter has been well established empirically, its dynamical origins remain mysterious. Indeed, even the most general considerations of symmetries in QCD do not provide much insight in this case. Similarly, the weak decays of strange baryons do not appear to respect the symmetries of QCD. The long standing $S$-wave/ $P$-wave discrepancy, wherein a simultaneous accounting for the parity conserving and parity violating amplitudes evades the grasp of chiral dynamics, points to a breakdown of QCD symmetries in the hadronic weak interaction (HWI). Similar issues also arise in weak radiative decays of polarized hyperons, which display PV asymmetries that should vanish in the limit of exact $S U(3)$ symmetry and that are unnaturally large from the standpoint of naive $S U(3)$ breaking expectations.

These issues pertaining to $\Delta S=1 \mathrm{HWIs}$ have been studied and reviewed extensively elsewhere, so we do not treat them in detail here (see, e.g., Refs. [477448] and references therein). We note, however, that they pose a more general question: are the apparent failures of QCD symmetries in strangeness

\footnotetext{
${ }^{23}$ Large CVC breaking effects would also be relevant in the context of the NuTeV discrepancy discussed in Section 2.1434 .
} 
changing HWIs due to the presence of a strange quark with mass close to the QCD scale, or do they reflect poorly understood dynamics at a more fundamental level? In order to address this question, one would ideally study the $\Delta S=0 \mathrm{HWI}$ - for which one expects strange quark effects to be sub-dominant — with the same level of intensity as one has scrutinized the $\Delta S=1$ sector. In practice, doing so has presented challenges of its own. As we outline below, however, recent experimental and theoretical developments may have put this field on a new and more promising footing.

Because the parity conserving $\Delta S=0 \mathrm{HWI}$ is masked at low energies by much larger strong and electromagnetic effects, one must isolate the former by studying PV processes. Historically, such studies have been carried out largely in nuclei, where various features of nuclear structure may amplify tiny PV effects. One generically expects the size of low energy, hadronic PV observables to be of order $G_{F} F_{\pi}^{2} \sim 10^{-7}$, thereby making their observation an enormously challenging task. Indeed, only two PV observables of this magnitude in the more pristine few-body system have been measured: $A_{L}^{p p}$, the longitudinal analyzing power in polarized proton-proton scattering [449 455], and the corresponding analyzing power in elastic $\vec{p} \alpha$ scattering, $A_{L}^{p \alpha}$ [456 457].

The remaining hadronic PV observables have involved nuclei that enhance the effects by several orders of magnitude. The largest effects have been observed in the scattering of polarized, epithermal neutrons from ${ }^{139} \mathrm{La}$, where a $\sim 10 \%$ asymmetry was seen [458 460],

$$
A_{z}=(9.55 \pm 0.35) \times 10^{-2}
$$

Unfortunately, the theoretical interpretation of measurements involving heavy nuclei is difficult, since one cannot perform ab initio nuclear structure computations for such systems. A somewhat more tractable situation arises in the case of the lighter $S-D$ shell nuclei, such as ${ }^{18} \mathrm{~F}$. Here, fortuitous near degeneracies of opposite parity states can enhance the magnitude of parity mixing in the nuclear wavefunction by factors of ten or more. When probed electromagnetically, these large parity mixing effects can be additionally amplified since the parity mixing allows contributions from large, parity forbidden multipole matrix elements to the transition amplitudes of interest. The resulting observables can be as large as a few $\times 10^{-4}$. For example, one has for the directional asymmetry in the $\gamma$ decay of polarized ${ }^{19} \mathrm{~F}$ from its first excited state,

$$
A_{\gamma}\left({ }^{19} \mathrm{~F}\right)=-(8.5 \pm 2.6) \times 10^{-5} \text { [461], } \quad A_{\gamma}\left({ }^{19} \mathrm{~F}\right)=-(6.8 \pm 1.8) \times 10^{-5} \text { [662, 463, }
$$

for the circular polarization in the $\gamma$ decay of excited ${ }^{21} \mathrm{Ne}$,

$$
P_{\gamma}\left({ }^{21} \mathrm{Ne}\right)=(24 \pm 24) \times 10^{-4} \text { 464], } \quad P_{\gamma}\left({ }^{21} \mathrm{Ne}\right)=(3 \pm 16) \times 10^{-4} \text { [465] }
$$

or for the circular polarization in the $\gamma$ decay of ${ }^{18} \mathrm{~F}$ (in units of $10^{-4}$ ),

$$
P_{\gamma}\left({ }^{18} \mathrm{~F}\right)=-7 \pm 20 \text { [466], } \quad P_{\gamma}\left({ }^{18} \mathrm{~F}\right)=3 \pm 6 \text { [467], } \quad P_{\gamma}\left({ }^{18} \mathrm{~F}\right)=-10 \pm 18 \text { [468], } \quad P_{\gamma}\left({ }^{18} \mathrm{~F}\right)=2 \pm 6 \text { [66]. }
$$

Note that the $P_{\gamma}$ results are consistent with zero. However, owing to the nuclear enhancements expected in ${ }^{21} \mathrm{Ne}$ and ${ }^{18} \mathrm{~F}$, measurements at the $10^{-4}$ level can probe $\mathcal{O}\left(10^{-7}\right)$ strangeness conserving HWIs.

Given the short range $(\sim 0.002 \mathrm{fm})$ of the HWI, it is improbable that direct exchange of $W$ and $Z$ bosons between nucleons are responsible for the PV effects observed in these nuclei. Indeed, the repulsive hard core of the strong $N N$ interaction that becomes dominant below $0.4 \mathrm{fm}$ highly suppresses direct $W$ and $Z$ exchange. Consequently, theorists have conventionally relied upon a meson exchange framework for describing the PV $N N$ interaction. In this picture, the PV HWI resides within one of the two meson-nucleon vertices appearing in the meson exchange amplitude (the second vertex involves a purely strong, parity conserving interaction). Moreover, the Compton wavelength of the lightest pseudoscalar and vector mesons is sufficient to overcome the short range repulsion of the $N N$ interaction. Thus, one 
has a physically natural mechanism for understanding the observed PV effects in nuclei, and one that is consistent with the long standing framework for understanding the strong $N N$ interaction.

Unfortunately, the application of this picture to nuclear PV entailed the reliance on a number of model approximations — not all of which are well controlled - and resulted in a less than successful phenomenology. The first approximation has been to severely truncate the spectrum of exchanged mesons, retaining only the $\pi, \rho$, and $\omega$. The resulting model for the PV $N N$ potential then depends on seven, a priori unknown PV meson-nucleon couplings, $h_{M}^{I}$, that appear in the PV $N N$ potential,

$$
\begin{aligned}
\mathcal{H}_{\mathrm{PV}}= & \frac{h_{\pi}^{1}}{\sqrt{2}} \bar{N}(\tau \times \pi)_{3} N-\bar{N}\left(h_{\rho}^{0} \tau \cdot \rho^{\mu}+h_{\rho}^{1} \rho_{3}^{\mu}+\frac{h_{\rho}^{2}}{2 \sqrt{6}}\left(3 \tau_{3} \rho_{3}^{\mu}-\tau \cdot \rho^{\mu}\right)\right) \gamma_{\mu} \gamma_{5} N \\
& -\bar{N}\left(h_{\omega}^{0} \omega^{\mu}+h_{\omega}^{1} \tau_{3} \omega^{\mu}\right) \gamma_{\mu} \gamma_{5} N+h_{\rho}^{\prime 1} \bar{N}\left(\tau \times \rho^{\mu}\right)_{3} \frac{\sigma_{\mu \nu} k^{\nu}}{2 m_{N}} \gamma_{5} N .
\end{aligned}
$$

Here, the superscripts in the $h_{M}^{I}$ denote the isospin $(I=0,1,2)$ of the corresponding meson-nucleon interaction. The longest range effect that arises from the exchange of charged pions is characterized by a single PV Yukawa coupling, $h_{\pi}^{1}$.

The goal of experiment has been to provide a self-consistent determination of these couplings. In doing so, one has relied upon many-body nuclear calculations in order to relate the PV $N N$ model potential to the PV observables. This portion of the analysis thereby entails a second level of approximation associated with the nuclear many-body problem. In some cases, such as $P_{\gamma}\left({ }^{18} \mathrm{~F}\right)$, one may attempt to constrain the nuclear theory uncertainties by calibrating the parity mixing matrix elements to those of the two-body axial charge operator that contribute to the analog forbidden $\beta$ decays. This semi-empirical approach, however, is not feasible in all cases. A recent example involves the anapole moment of ${ }^{133} \mathrm{Cs}$ (see Section 2.3), where the state of the art involves a shell model computation using a truncated model space. In fact, a comparison of the ${ }^{133} \mathrm{Cs}$ analysis with that of ${ }^{18} \mathrm{~F}$ presents a particularly puzzling phenomenological inconsistency. While the analysis of the $P_{\gamma}\left({ }^{18} \mathrm{~F}\right)$ measurements imply a value for $h_{\pi}^{1}$ consistent with zero (assuming the DDH ranges for $h_{\rho}^{1}$ and $h_{\omega}^{1}$ discussed below) [470],

$$
-0.6 \lesssim h_{\pi}^{1}-0.11 h_{\rho}^{1}-0.19 h_{\omega}^{1} \lesssim 1.2
$$

a combination of $A_{L}^{p p}$ results and the ${ }^{133} \mathrm{Cs}$ anapole moment suggests that $h_{\pi}^{1}$ is considerably larger. Whether the origin of this discrepancy lies with experiment, the nuclear many-body theory, or the adoption of an inadequate model for the PV $N N$ potential is unknown at present. One expects that a precise measurement of $A_{\gamma}(\vec{n} p \rightarrow d \gamma)$, the photon asymmetry in the capture of polarized neutrons on hydrogen that is underway at LANSCE 471472, will shed new light on this question.

Even if one were able to extract a self-consistent set of values for the $h_{M}^{I}$ from experiment, their interpretation in terms of the underlying weak quark-quark interaction would remain problematic. It is not entirely clear, for example, that the $h_{M}^{I}$ represent the couplings of the physical mesons to the nucleon rather than some effective parameters that include the contributions from a tower of heavier meson exchanges. Under the assumption that the $h_{M}^{I}$ do represent physical meson-nucleon couplings, Desplanques, Donoghue, and Holstein (DDH) used symmetry arguments and quark model computations to predict values for the PV couplings from the underlying $\Delta S=0 \mathrm{PV}$ quark-quark interactions [473]. The so-called DDH "reasonable ranges" and their updates 474475] (see Table 11) have become the benchmark for comparison with experiment, and their latitude reflect the theoretical strong interaction uncertainties entering the treatment of HWIs. Even the symmetry arguments used by DDH may not be entirely well founded. For example, the effects of chiral symmetry breaking on the value of $h_{\pi}^{1}$ not included in the DDH treatment - may be anomalously large [140.

These theoretical and phenomenological issues triggered a reformulation of the hadronic PV problem [76 478. The basic thrust of that work was to recast the PV $N N$ interaction into the framework of an EFT, thereby eliminating the dependence on the model dependent meson exchange picture. The 
Table 11: Weak, PV meson-nucleon couplings as calculated in Refs. 473 475]. All numbers are quoted in units of the "sum rule" value $g_{\pi}=3.8 \times 10^{-8}$ 473].

\begin{tabular}{|c|c|c|c|c|}
\hline Coupling & DDH Reasonable Range [473] & DDH Best Value [473] & DZ [474] & FCDH [475] \\
\hline$h_{\pi}^{1}$ & $0 \rightarrow+30$ & +12 & +3 & +7 \\
$h_{\rho}^{0}$ & $+30 \rightarrow-81$ & -30 & -22 & -10 \\
$h_{\rho}^{1}$ & $-1 \rightarrow 0$ & -0.5 & +1 & -1 \\
$h_{\rho}^{2}$ & $-20 \rightarrow-29$ & -25 & -18 & -18 \\
$h_{\omega}^{0}$ & $+15 \rightarrow-27$ & -5 & -10 & -13 \\
$h_{\omega}^{1}$ & $-5 \rightarrow-2$ & -3 & -6 & -6 \\
\hline
\end{tabular}

use of EFT allows one to expand $\mathcal{H}_{\mathrm{PV}}$ in the most general set of PV operators, and systematically classifying them in powers of $Q / \Lambda_{\chi}$, where $Q \ll 1 \mathrm{GeV}$ is either the pion mass or a typical momentum transfer associated with the $N N$ interaction. Doing so allows one to truncate the expansion at a given order in $Q / \Lambda_{\chi}$ with a reasonable expectation for the magnitude of the truncation error. The coefficients of the operators are left as a priori unknown "low energy constants" (LECs) that are to be determined from experiment. This approach is model independent, since, to a given order in the expansion, one includes all operators consistent with the symmetries of the underlying EW and strong interactions. In essence, the meson exchange model dictates certain relations among these operators — relations that may not, in fact, be consistent with experiment.

For very low energies, one may treat the pion as heavy and integrate it out from the EFT, leaving only short range, four-nucleon operators in $\mathcal{H}_{\mathrm{PV}}$. Through $\mathcal{O}\left(Q / \Lambda_{\chi}\right)$, there exist only five independent operators and, thus, only five independent LECs that must be taken from experiment,

$$
\lambda_{s}^{p p}, \quad \lambda_{s}^{p n}, \quad \lambda_{s}^{n n}, \quad \lambda_{t}, \text { and } \rho_{t} .
$$

For higher energy processes, for which $E \gtrsim m_{\pi}$, the pion must be treated as a dynamical degree of freedom and additional operators appear in the EFT. In this case, the leading contribution in $\mathcal{H}_{\mathrm{PV}}$ arises at $\mathcal{O}\left(\Lambda_{\chi} / Q\right)$ and is the same long range pion exchange operator containing $h_{\pi}^{1}$ in Eq. (185).

The future program for hadronic PV that is laid out in Refs. 476-478] involves performing a set of few-body measurements to determine the coefficients of the operators appearing in the EFT. In the case of the EFT without pions, the constants (187) could be obtained by combining the $\overrightarrow{p p}$ and $\vec{p} \alpha$ results with three additional observables: the circular polarization, $P_{\gamma}$, and photon asymmetry, $A_{\gamma}$, in radiative $n p$ capture and the rotation of neutron spin as it passes through a gas of ${ }^{4} \mathrm{He}, d \phi^{n \alpha} / d z$. Using somewhat idealized few-body wave-functions, Refs. 476 478 obtained approximate relations between the PV constants and these observables,

$$
\begin{aligned}
m_{N} \lambda_{s}^{p p} & =-1.22 A_{L}^{p p}(45 \mathrm{MeV}) \\
m_{N} \rho_{t} & =-9.35 A_{\gamma}(n p \rightarrow d \gamma) \\
m_{N} \lambda_{s}^{p n} & =1.6 A_{L}^{p p}(45 \mathrm{MeV})-3.7 A_{L}^{p \alpha}(46 \mathrm{MeV})+37 A_{\gamma}(n p \rightarrow d \gamma)-2 P_{\gamma}(n p \rightarrow d \gamma) \\
m_{N} \lambda_{t} & =0.4 A_{L}^{p p}(45 \mathrm{MeV})-0.7 A_{L}^{p \alpha}(46 \mathrm{MeV})+7 A_{\gamma}(n p \rightarrow d \gamma)+P_{\gamma}(n p \rightarrow d \gamma) \\
m_{N} \lambda_{s}^{n n} & =0.83 \frac{d \phi^{n \alpha}}{d z}-33.3 A_{\gamma}(n p \rightarrow d \gamma)-0.69 A_{L}^{p p}(45 \mathrm{MeV})+1.18 A_{L}^{p \alpha}(46 \mathrm{MeV})-1.08 P_{\gamma}(n p \rightarrow d \gamma)
\end{aligned}
$$

Although a more precise version of these relations will require the use of more sophisticated few-body methods, Eqs. (188) provide a rough guide for a future experimental program. In the case of the EFT 
with explicit pions, additional experiments, such as radiative $n d$ capture or $n p$ spin rotation, will be needed in order to determine all of the PV parameters.

Once such a program is completed, the LECs, $h_{\pi}^{1}, \lambda_{s}^{p p}$, etc., would provide model independent input for theoretical analyses. One direction would involve first principles studies that would address the fundamental question of QCD symmetries and the HWI outlined above. For example, are the magnitudes of the various LECs consistent with what one expects based on these symmetry considerations? Or, does one find surprising enhancements as in the $\Delta S=1$ sector? Alternately, the PV $N N$ interaction, $\mathcal{H}_{\mathrm{PV}}$, could be used to re-analyze the PV observables in many-body systems. To the extent that one is able to understand the latter using the more "primordial" PV $N N$ interaction derived from EFT and few body experiments, one would have some confidence of the applicability of the EFT to complex nuclei - a situation that might open the way to applying EFT methods to other weak processes, such as $0 \nu \beta \beta$ decay discussed in Section 5.6.

\subsection{Probes of Nucleon Strangeness}

As noted in the foregoing discussion, the strange quark — with its mass of order $\Lambda_{\mathrm{QCD}}$ — presents a variety of theoretical challenges from the standpoint of HWIs. It is a similarly problematic object for those seeking to understand the structure of hadrons. Its mass is not sufficiently heavy to apply heavy quark effective theory, and it may not be sufficiently light to apply chiral dynamics with a high degree of confidence. In the simplest quark model description of the lowest lying non-strange baryons, the strange quark does not appear as a constituent degree of freedom. On the other hand, there have been indications that it contributes substantially to the nucleon mass and possibly to the nucleon spin. For these reasons, there has been considerable interest within the hadron structure community over the past decade to probe other aspects of strange quarks in the nucleon, in the hope of gaining new insights into the non-perturbative strong interaction.

In this regard, the weak NC interaction between leptons and quarks has provided a new tool to study strange quarks. The basic idea, first proposed by Kaplan and Manohar [479, is that the hadronic vector weak NC contains a different linear combination of the various quark currents, $\bar{q} \gamma_{\mu} q(q=u, d, s)$ than the electromagnetic current. Similarly, the hadronic axial vector NC contains a term involving $\bar{q} \gamma_{\mu} \gamma_{5} q$ that is not simply related via an $S U(3)$ flavor rotation to the charge changing weak currents relevant to weak decays or CC lepton-nucleon scattering. By performing measurements of the hadronic weak NC form factors, one obtains additional information that allows one to experimentally separate the up-, down-, and strange quark vector and axial vector current matrix elements in the nucleon. Then one can in principle determine the role played by the strange quark in the nucleon's electromagnetic and spin structure. For example, in the case of the vector currents, one has for the proton [136 480],

$$
G_{E, M}^{N C}\left(Q^{2}\right)=2 Q_{W}(p) G_{E, M}^{p}\left(Q^{2}\right)+2 Q_{W}(n) G_{E, M}^{I=0}\left(Q^{2}\right)-G_{E, M}^{s}\left(Q^{2}\right),
$$

where $Q_{W}(p)=1 / 2-2 \sin ^{2} \theta_{W}$ and $Q_{W}(n)=-1 / 2$ are the tree level proton and neutron weak charges (see Section 2.2) and the $G_{E, M}^{a}$ are the Sachs electric $(E)$ and magnetic $(M)$ proton $(a=p)$ and neutron $(a=n)$ electromagnetic and strange quark $(a=s)$ form factors. Since one has experimental information on $G_{E, M}^{p, n}\left(Q^{2}\right)$ from parity conserving electron scattering, measurements of $G_{E, M}^{N C}\left(Q^{2}\right)$ with parity violating electron scattering (PVES) effectively provide determinations of $G_{E, M}^{s}\left(Q^{2}\right)$. Note that at very low momentum transfer $G_{E}^{n}\left(Q^{2}\right)$ and $G_{E}^{s}\left(Q^{2}\right)$ vanish linearly with $Q^{2}$, so that a measurement of $G_{E}^{N C}\left(Q^{2}\right)$ at sufficiently low $Q^{2}$ provides a determination of the proton's weak charge.

A substantial program involving PVES has been generated by these ideas. Since this program has been extensively reviewed in Refs. [136] and [481,484, we give only a brief summary here. The PVES program involves measuring the PV helicity asymmetry for scattering longitudinally polarized electrons 
Table 12: Present (upper part) and future (lower part) strange quark form factor (FF) determinations with PVES and neutrino scattering. Statistical and systematic errors have been combined in quadrature.

\begin{tabular}{|c|c|c|c|c|c|}
\hline Experiment & Lepton & Target & FF Combination & $\left|Q^{2}\right|(\mathrm{GeV})^{2}$ & Result \\
\hline $\begin{array}{l}\text { SAMPLE } 484485] \\
\text { HAPPEX } 486] \\
\text { A4 [490] } \\
\text { BNL E734 [105 493] }\end{array}$ & $\begin{array}{c}e \\
e \\
e \\
\nu_{\mu}, \bar{\nu}_{\mu} \\
\nu_{\mu}, \bar{\nu}_{\mu} \\
\nu_{\mu}, \bar{\nu}_{\mu}\end{array}$ & $\begin{array}{c}p,{ }^{2} \mathrm{H} \\
p \\
p \\
{ }^{12} \mathrm{C} \\
{ }^{12} \mathrm{C} \\
{ }^{12} \mathrm{C}\end{array}$ & $\begin{array}{c}G_{M}^{s} \\
G_{E}^{s}+0.392 G_{M}^{s} \\
G_{E}^{s}+0.225 G_{M}^{s} \\
0.2 \rightarrow 1.2 \\
0.2 \rightarrow 1.2 \\
0.2 \rightarrow 1.2\end{array}$ & $\begin{array}{c}0.1 \\
0.477 \\
0.230 \\
G_{A}^{s}(0) \\
G_{M}^{s}(0) \\
m_{N}^{2} d G_{E}^{s}(0) / d Q^{2}\end{array}$ & $\begin{array}{c}0.37 \pm 0.34 \\
0.014 \pm 0.022 \\
0.039 \pm 0.034 \\
-0.21 \pm 0.10 \\
-0.40 \pm 0.72 \\
-0.57 \pm 0.62\end{array}$ \\
\hline $\begin{array}{l}\text { HAPPEX } 487,488 \\
\text { HAPPEX } 488,489 \\
\text { GØ } 491492\end{array}$ & $\begin{array}{c}e \\
e \\
e \\
e \\
e \\
e \\
e \\
e \\
\nu_{\mu}\end{array}$ & $\begin{array}{c}p \\
{ }^{4} \mathrm{He} \\
p \\
p \\
p \\
p \\
p \\
p \\
{ }^{12} \mathrm{C}\end{array}$ & $\begin{array}{c}G_{E}^{s}+0.08 G_{M}^{s} \\
G_{E}^{s} \\
G_{E}^{s} \\
G_{E}^{s} \\
G_{E}^{s} \\
G_{M}^{s} \\
G_{M}^{s} \\
G_{M}^{s} \\
0.5 \rightarrow 1.0\end{array}$ & $\begin{array}{c}0.01 \\
0.01 \\
0.300 \\
0.500 \\
0.800 \\
0.300 \\
0.500 \\
0.800 \\
G_{A}^{s}\end{array}$ & $\begin{array}{l} \pm 0.01 \\
\pm 0.015 \\
\pm 0.112 \\
\pm 0.073 \\
\pm 0.051 \\
\pm 0.031 \\
\pm 0.033 \\
\pm 0.034 \\
\pm 0.04\end{array}$ \\
\hline
\end{tabular}

from a hadronic target. For elastic scattering one has,

$$
A_{\mathrm{LR}}=\frac{d \sigma_{+}-d \sigma_{-}}{d \sigma_{+}+d \sigma_{-}}=\frac{G_{F} Q^{2}}{4 \sqrt{2} \pi \alpha}\left[Q_{W}+F\left(Q^{2}, \theta\right)\right],
$$

where $d \sigma_{+(-)}$is the cross section for scattering electrons with positive (negative) helicity; $F\left(Q^{2}, \theta\right)$ is a term that depends on the target's electromagnetic, axial vector, and strange quark form factors; $Q^{2}=|\vec{q}|^{2}-q_{0}^{2}$; and $\theta$ is the laboratory scattering angle. The dependence on $Q^{2}$ and $\theta$ can be exploited to isolate the dependence on various form factor components (e.g., electric, magnetic, and axial).

A variety of measurements of the vector NC form factors are being carried out at MIT-Bates, the MAMI facility in Mainz, and at Jefferson Lab. A summary of the present and future strange quark form factor determinations from these experiments is given in Table 12 .

Theoretical uncertainties in the EW radiative corrections preclude the use of PVES for a determination of $G_{A}^{s}$ [139]. The cross section for neutrino-nucleon scattering is free from such uncertainties, however, and thus provides a theoretically clean means of obtaining this form factor. Several neutrino scattering measurements have been used. The analysis of these measurements is complicated by the kinematics of neutrino NC scattering, for which one detects recoil nucleons rather than the outgoing lepton. The experimental cross sections are, thus, effective integrals over $Q^{2}$, so only the form factor for an average $<Q^{2}>$ or fits to form factor moments can be quoted. The values shown in Table 12 for the BNL E734 experiment [105] are taken from a reanalysis by Garvey et al. 493] in which the form factors and their slopes at $Q^{2}=0$ were fit to the data (for other reanalyses, see Refs. [495 496]). Note that the experiments performed to date have entailed the use of QE neutrino-nucleus scattering, which introduces some small degree of nuclear model dependence. A future measurement at Fermilab (FINeSSE) has been proposed, but it is yet to be approved for running [494. For a review of the theoretical hadron structure implications of the current experimental results, see e.g. Ref. [484. 
In addition to the use of PVES to probe strangeness, it may also provide a probe of various aspects of nucleon structure. In particular, the PAVEX experiment at Jefferson Lab will use elastic PVES from ${ }^{208} \mathrm{~Pb}$ to measure the Fourier transform of the neutron distribution in that nucleus [180. In contrast to the photon, the $Z$ couples preferentially to neutrons rather than protons at low momentum transfer, making it a particularly powerful probe of neutron properties in the nuclear medium. In the past, strongly interacting probes, such as pions and protons, have been used in attempts to determine such neutron properties. In contrast, the purely leptonic probe of PVES provides a theoretically cleaner means of studying the neutron distribution.

\section{Summary and Outlook}

The next decade promises to be a time of discovery in the field of EW physics. With the operation of the Large Hadron Collider at CERN, one expects to discover at least one Higgs boson and to find evidence for other new particles, such as the superpartners of SUSY or additional light gauge bosons of grand unified theories. As outlined in this article, progress on the high energy collider front is being matched by new strides in the study of low energy weak interactions involving light quarks and leptons. The precision of experiments in this arena are making them sensitive to NP at the TeV scale and beyond in ways that complement the reach of colliders. From our perspective, several aspects of the future program of low energy studies merit emphasizing:

- Neutrino properties. Now that neutrino oscillations have provided the clearest experimental "smoking gun" for physics beyond the SM, it is essential to map out the characteristics of neutrinos and their interactions in as detailed a manner as possible. In particular, one would like to know the scale of neutrino mass, whether there exist more than three generations of light neutrinos, whether the neutrino is a Majorana particle, and whether the neutrino mixing angle $\theta_{13}$ is sufficiently large to lead to significant $\mathrm{CP}$ violating effects in the lepton sector. From this standpoint, more precise measurements of tritium $\beta$ decay and neutrinoless double $\beta$ decay, along with 1-2 $\mathrm{km}$ reactor-based studies and long baseline experiments should provide important insights.

- Electric Dipole Moments. The prospects that one or more EDM searches will find a non-zero result during the next decade are strong. Breakthroughs in experimental techniques for probing the EDMs of the electron, neutron, and neutral atoms are pushing the sensitivity to a range expected from models of EWB. Complementary efforts are also underway involving the muon and deuteron.

- Weak mixing. The running of $\sin ^{2} \theta_{W}$ below the $Z$ pole remains a largely untested feature of the SM. The PV Møller experiment at SLAC will test this running precisely by measuring $\sin ^{2} \theta_{W}$ at a specific low energy value of the momentum transfer, and the PV ep experiment at Jefferson Lab will provide a complementary test at the same scale. Together, these experiments, along with the results from APV and neutrino-nucleus deep inelastic scattering, begin to map out the scale dependence of $\sin ^{2} \theta_{W}$ and either provide indications of NP or lead to stringent constraints on deviations from the SM. Additional tests of low scale weak mixing, however, would be ideal.

- Weak decays of light quarks. The weak decays of light quarks are being studied with increasingly high precision on a number of fronts. An issue that clearly calls for resolution is that of the unitarity of the CKM matrix. In this respect, new experiments in neutron $\beta$ decay and new studies of kaon leptonic decays are underway, as are measurements of nuclear and pion $\beta$ decay. Open theoretical questions involving the effects of strong interactions in EW radiative corrections and the momentum dependence of kaon form factors also require further scrutiny. Should the long 
standing deviation from unitarity persist, light quark decays would provide important constraints on scenarios for NP.

- Muon physics. In the near future, the TWIST experiment will produce a substantially more precise determination of the Michel parameters, by up to factors of $\sim 60$ in some cases. Similarly, the MECO experiment at Brookhaven will improve the sensitivity to $\mu \rightarrow e$ conversion by a factor of $10^{4}$, providing a new window to lepton flavor violation at the GUT scale in SUSY models or the TeV scale in other scenarios. Measurements of the muon anomalous magnetic moment and electric dipole moment provide complementary probes of physics at the TeV scale. In particular, the recent determination of $(g-2)_{\mu}$ has provided a tantalizing hint at SUSY, and a more precise measurement would complement future collider searches for superpartners.

Throughout our discussion of these studies, an important theme has been the complementarity of low energy and high energy probes. Just as in the case of the top quark, where the comparison of indirect constraints from precision measurements and direct measurements at the Tevatron provided an important test of the self-consistency of the SM, similar comparisons will be essential in the future for testing whatever the "new Standard Model" is to be. In some cases, such as $0 \nu \beta \beta$ decay, electric dipole moments, and $\mu \rightarrow e(\gamma)$, low energy studies can provide a more direct and more powerful probe of certain aspects of NP than one may hope to achieve with colliders during the next decade.

At the same time, many of these low energy probes require having in hand a sufficiently clear theoretical picture. In the case of quantities such as $(g-2)_{\mu}$, for which the SM predicts a non-zero result, obtaining a sufficiently reliable treatment of strong interaction contributions is essential if one is going to make strong inferences about NP from any report of a deviation. In this respect, there exists considerable room for future interplay between EW and QCD theory. More generally, it is important to emphasize the role of theory in assembling the information obtained from the broad range of studies - both low and high energy - and identifying which of the many candidates for NP provides the most coherent account.

In summary, our view is that the field of low energy tests of the weak interaction will continue to be an exciting one during the next decade, providing for a rich and fruitful interplay between particle, nuclear, and atomic physicists — in both experiment and theory.

\section{Acknowledgments}

We gratefully acknowledge the receipt of information from, and useful discussions with, V. Cirigliano, D. DeMille, B. Filippone, N. Fortson, S. Gardner, J. Hardy, P. Herczeg, D. Hertzog, B. Holstein, R. Holt, K. Kumar, A. Kurylov, S. Lamoreaux, P. Langacker, F. Maas, W. Marciano, J. Martin, L. Orozco, C. Peña-Garay, M. Pitt, M. Pospelov, M. Romalis, J. Stalnaker, S. Su, R. Tribble, and P. Vogel. We also thank the Institute for Nuclear Theory at the University of Washington, where part of this work was carried out. MR-M thanks the Department of Theoretical Physics at the Physics Institute at UNAM for hospitality during the completion of this review. This work was supported in part by U.S. Department of Energy contracts DE-FG02-00ER41146, DE-FG03-02ER41215, and DE-FG03-00ER41132, by the National Science Foundation Award PHY00-71856, by CONACYT (México) contract 42026-F, and by DGAPA-UNAM contract PAPIIT IN112902.

\section{References}

[1] S. Weinberg, Phys. Rev. Lett. 19 (1967) 1264

[2] A. Salam, p. 367 of Ref. 3] 
[3] Elementary Particle Theory, N. Svartholm (ed.), (Almquist and Wiksells, Stockholm, 1969)

[4] F. Wilczek, Phys. Rev. Lett. 40 (1978) 279

[5] Y. Nir, preprint hep-ph/0109090, lectures at 55th Scottish Universities Summer School in Physics:

Heavy Flavor Physics, 7-23 Aug 2001, St. Andrews, Scotland, C. Davies and S. M. Playfer (eds.)

[6] R. Fleischer, Phys. Rep. 370 (2002) 537

[7] Y. Nir, Nucl. Phys. Proc. Suppl. B 117 (2003) 111

[8] T. Hurth, Rev. Mod. Phys. 75 (2003) 1159

[9] M. Battaglia et al., preprint hep-ph/0304132

[10] G. Isidori, preprint hep-ph/0401079, talk presented at Beauty 2003

[11] W. J. Marciano and A. Sirlin, Phys. Rev. D 22 (1980) 2695

[12] A. Czarnecki and W. J. Marciano, Int. J. Mod. Phys. A 15 (2000) 2365

[13] C. S. Wood et al., Science 275 (1997) 1759

[14] S. C. Bennett and C. E. Wieman, Phys. Rev. Lett. 82 (1999) 2484

[15] NuTeV Collaboration: G. P. Zeller et al., Phys. Rev. Lett. 88 (2002) 091802

[16] SLAC-E-158 Collaboration: P. L. Anthony et al., preprint hep-ex/0312035

[17] Qweak Collaboration: D. Armstrong et al., proposal JLAB-E-02-020, http://www.jlab.org/exp_prog/proposals/02/PR02-020.pdf

[18] F. E. Close and Q. Zhao, preprint hep-ph/0403159

[19] N. Cabibbo, Phys. Rev. Lett. 10 (1963) 531

[20] M. Kobayashi and T. Maskawa, Prog. Theor. Phys. 49 (1973) 652

[21] CDF Collaboration: F. Abe et al., Phys. Lett. B 74 (1995) 2626

[22] DONUT Collaboration: K. Kodama et al., Phys. Lett. B 504 (2001) 218

[23] J. Erler, preprint hep-ph/0005084, in Physics At Run II: QCD And Weak Boson Physics Workshop: 2nd General Meeting, 3-4 Jun 1999, Batavia, IL, U. Baur et al. (eds.)

[24] ALEPH, DELPHI, L3, and OPAL Collaborations, LEP Electroweak Working Group and SLD Heavy Flavor Group: D. Abbaneo et al., preprint hep-ex/0212036

[25] OPAL Collaboration: K. Ackerstaff et al., Z. Phys. C 76 (1997) 387

[26] DELPHI Collaboration: P. Abreu et al., Eur. Phys. J. C 14 (2000) 613

[27] SLD Collaboration: K. Abe et al., Phys. Rev. Lett. 85 (2000) 5059

[28] SLD Collaboration: K. Abe et al., Phys. Rev. Lett. 84 (2000) 5945

[29] SLD Collaboration: K. Abe et al., Phys. Rev. Lett. 86 (2001) 1162

[30] A. G. Holzner, preprint hep-ex/0208045, talk presented at BEACH 2002

[31] SLD Collaboration: K. Abe et al., Phys. Rev. Lett. 78 (1997) 17

[32] DØ Collaboration: B. Abbott et al., Phys. Rev. D 58 (1998) 052001

[33] CDF Collaboration: F. Abe et al., Phys. Rev. Lett. 82 (1999) 271

[34] UA2 Collaboration: J. Alitti et al., Phys. Lett. B 276 (1992) 354

[35] DØ Collaboration: B. Abbott et al., Phys. Rev. Lett. 84 (2000) 222

[36] CDF Collaboration: T. Affolder et al., Phys. Rev. D 64 (2001) 052001

[37] M. C. Smith and S. S. Willenbrock, Phys. Rev. Lett. 79 (1997) 3825

[38] J. Erler, Phys. Rev. D 59 (1999) 054008

[39] J. Erler and M. Luo, Phys. Lett. B 558 (2003) 125

[40] J. Erler, Phys. Rev. D 63 (2001) 071301 
[41] DØ Collaboration: V.M. Abazov et al., Nature 429 (2004) 638

[42] J. Erler and P. Langacker, preprint hep-ph/0407097, in Ref. [43]

[43] Particle Data Group: S. Eidelman et al., Phys. Lett. B 592 (2004) 1

[44] J. R. Primack, Nucl. Phys. Proc. Suppl. 124 (2003) 3

[45] G. 't Hooft and M. J. Veltman, Ann. Poincare Phys. Theor. A 20 (1974) 69

[46] J. Wess and B. Zumino, Phys. Lett. B 49 (52) 1974, Nucl. Phys. B 70 (1974) 39 and 78 (1974) 1

[47] S. Dimopoulos and H. Georgi, Nucl. Phys. B 193 (1981) 150

[48] N. Sakai, Z. Phys. C 11 (1981) 153

[49] M. T. Grisaru, W. Siegel and M. Rocek, Nucl. Phys. B 159 (1979) 429

[50] W. Fischler, H. P. Nilles, J. Polchinski, S. Raby and L. Susskind, Phys. Rev. Lett. 47 (1981) 757

[51] E. Witten, Nucl. Phys. B 188 (1981) 513

[52] S. Dimopoulos, S. Raby and F. Wilczek, Phys. Rev. D 24 (1981) 1681

[53] L. E. Ibañez and G. G. Ross, Phys. Lett. B 105 (1981) 439

[54] U. Amaldi et al., Phys. Rev. D 36 (1987) 1385

[55] M. Carena, M. Quiros and C. E. Wagner, Nucl. Phys. B 461 (1996) 407

[56] H. E. Haber, R. Hempfling and A. H. Hoang, Z. Phys. C 75 (1997) 539

[57] S. Heinemeyer, W. Hollik and G. Weiglein, Eur. Phys. J. C 9 (1999) 343

[58] J. R. Espinosa and R. J. Zhang, Nucl. Phys. B 586 (2000) 3

[59] A. Brignole, G. Degrassi, P. Slavich and F. Zwirner, Nucl. Phys. B6311952002

[60] G. Degrassi et al., Eur. Phys. J. C 28 (2003) 133

[61] L. Girardello and M. T. Grisaru, Nucl. Phys. B 194 (1982) 65

[62] J. E. Kim and H. P. Nilles, Phys. Lett. B 138 (1984) 150

[63] A. Salam and J. Strathdee, Nucl. Phys. B 87 (1975) 85

[64] P. Fayet, Nucl. Phys. B 90 (1975) 104

[65] P. Fayet and S. Ferrara, Phys. Rep. 32 (1977) 249

[66] M. F. Sohnius, Phys. Rep. 128 (1985) 39

[67] H. P. Nilles, Phys. Rep. 110 (1984) 1

[68] H. E. Haber and G. L. Kane, Phys. Rep. 117 (1985) 75

[69] E. Farhi and L. Susskind, Phys. Rep. 74 (1981) 277

[70] P. Langacker, Phys. Rep. 72 (1981) 185

[71] M. J. Duff, B. E. Nilsson and C. N. Pope, Phys. Rep. 130 (1986) 1

[72] M. B. Green, J. H. Schwarz and E. Witten, Superstring Theory, Vol. 1 and 2 (Cambridge Monographs On Mathematical Physics, 1987)

[73] J. L. Hewett and T. G. Rizzo, Phys. Rep. 183 (1989) 193

[74] M. Cvetič and P. Langacker, Phys. Rev. D 54 (1996) 3570

[75] J. Erler, Nucl. Phys. B 586 (2000) 73

[76] S. Bellucci, M. Lusignoli and L. Maiani, Nucl. Phys. B 189 (1981) 329

[77] D. Y. Bardin, P. K. Khristova and O. M. Fedorenko, Nucl. Phys. B 175 (1980) 435 and 197 (1982) 1

[78] Gargamelle Neutrino Collaboration: F. J. Hasert et al., Phys. Lett. B 46 (1973) 138

[79] C. H. Llewellyn Smith, Nucl. Phys. B 228 (1983) 205 
[80] E. A. Paschos and L. Wolfenstein, Phys. Rev. D 7 (1973) 91

[81] R. M. Barnett, Phys. Rev. D 14 (1976) 70

[82] H. Georgi and H. D. Politzer, Phys. Rev. D 14 (1976) 1829

[83] CDHS Collaboration: A. Blondel et al., Z. Phys. C 45 (1990) 361

[84] CHARM Collaboration: J. V. Allaby et al., Z. Phys. C 36 (1987) 611

[85] F. Perrier, p. 385 of Ref. [86]

[86] Precision Tests of the Standard Electroweak Model, P. Langacker (ed.), Advanced series on directions in high energy physics: 14 (World Scientific, Singapore, 1995)

[87] CCFR Collaboration: K. S. McFarland et al., Eur. Phys. J. C 1 (1998) 509

[88] G. A. Miller and A. W. Thomas, preprint hep-ex/0204007

[89] NuTeV Collaboration: G. P. Zeller et al., preprint hep-ex/0207052

[90] S. Kovalenko, I. Schmidt and J. J. Yang, Phys. Lett. B 546 (2002) 68

[91] S. Davidson, S. Forte, P. Gambino, N. Rius and A. Strumia, JHEP 0202 (2002) 037

[92] NuTeV Collaboration: M. Goncharov et al., Phys. Rev. D 64 (2001) 112006 , as quoted in Ref. 93

[93] S. Davidson, preprint hep-ph/0209316, talk presented at the NuFact02 Summer Institute

[94] NuTeV Collaboration: G. P. Zeller et al., Phys. Rev. D 65 (2002) 111103

[95] A. Bodek et al., Phys. Rev. Lett. 83 (1999) 2892

[96] B. A. Dobrescu and R. K. Ellis, preprint hep-ph/0310154

[97] S. Kretzer et al., preprint hep-ph/0312322

[98] A. Kurylov, M. J. Ramsey-Musolf and S. Su, Nucl. Phys. B 667 (2003) 321

[99] P. Langacker and M. Plümacher, Phys. Rev. D 62 (2000) 013006

[100] J. Erler and P. Langacker, Phys. Rev. Lett. 84 (2000) 212

[101] K. S. Babu and J. C. Pati, preprint hep-ph/0203029

[102] W. Loinaz, N. Okamura, T. Takeuchi and L. C. Wijewardhana, Phys. Rev. D 67 (2003) 073012

[103] J. M. Conrad, J. M. Link and M. H. Shaevitz, preprint hep-ex/0403048.

[104] A. K. Mann, p. 491 of Ref. 86]

[105] BNL-E-734 Collaboration: L. A. Ahrens et al., Phys. Rev. D 35 (1987) 785

[106] J. Panman, p. 504 of Ref. [86]

[107] S. Sarantakos, A. Sirlin and W. J. Marciano, Nucl. Phys. B 217 (1983) 84

[108] D. Y. Bardin and V. A. Dokuchaeva, Nucl. Phys. B 246 (1984) 221

[109] CHARM Collaboration: J. Dorenbosch et al., JournalZ. Phys. C415671989

[110] CHARM-II Collaboration: P. Vilain et al., Phys. Lett. B 335 (1994) 246

[111] BNL-E-734 Collaboration: L. A. Ahrens et al., Phys. Rev. D 41 (1990) 3297

[112] J. Erler and P. Langacker, p. 98 of Ref. [113]

[113] Particle Data Group: K. Hagiwara et al., Phys. Rev. D 66 (2002) 010001

[114] R. C. Allen et al., Phys. Rev. D 47 (1993) 11

[115] LSND Collaboration: L. B. Auerbach et al., Phys. Rev. D 63 (2001) 112001

[116] F. Reines, H. S. Gurr and H. W. Sobel, Phys. Rev. Lett. 37 (1976) 315

[117] P. Q. Hung and J. J. Sakurai, Phys. Lett. B 63 (1976) 295

[118] J. F. Wheater, Phys. Lett. B 105 (1981) 483

[119] W. J. Marciano and A. Sirlin, Phys. Rev. D 27 (1983) 552 and 29 (1984) 75 
[120] W. J. Marciano, p. 170 of Ref. 86

[121] E. Derman, Phys. Rev. D 7 (1973) 2755

[122] S. M. Berman and J. R. Primack, Phys. Rev. D 9 (1974) 2171

[123] SLAC-E-122 Collaboration: C. Y. Prescott et al., Phys. Lett. B 77 (1978) 347

[124] SLAC-E-122 Collaboration: C. Y. Prescott et al., Phys. Lett. B 84 (1979) 524

[125] R. N. Cahn and F. J. Gilman, Phys. Rev. D 17 (1978) 1313

[126] J. D. Bjorken, Phys. Rev. D 18 (1978) 3239

[127] L. Wolfenstein, Nucl. Phys. B 146 (1978) 477

[128] H. Fritzsch, Z. Phys. C 1 (1979) 321

[129] J. E. Kim, P. Langacker, M. Levine and H. H. Williams, Rev. Mod. Phys. 53 (1981) 211

[130] P. Q. Hung and J. J. Sakurai, Ann. Rev. Nucl. Part. Sci. 31 (1981) 375

[131] CERN-NA-004 Collaboration: A. Argento et al., Phys. Lett. B 120 (1983) 245

[132] W. Heil et al., Nucl. Phys. B 327 (1989) 1

[133] E. Hoffmann and E. Reya, Phys. Rev. D 18 (1978) 3230

[134] G. Feinberg, Phys. Rev. D 12 (1975) 3575

[135] J. D. Walecka, Nucl. Phys. A 285 (1977) 349

[136] M. J. Musolf et al., Phys. Rep. 239 (1994) 1

[137] P. A. Souder et al., Phys. Rev. Lett. 65 (1990) 694

[138] SAMPLE Collaboration: R. Hasty et al., Science 290 (2000) 2117

[139] M. J. Musolf and B. R. Holstein, Phys. Lett. B 242 (1990) 461

[140] S. L. Zhu, S. J. Puglia, B. R. Holstein and M. J. Ramsey-Musolf, Phys. Rev. D 62 (2000) 033008

[141] P. A. Souder, p. 599 of Ref. 86]

[142] J. Erler, A. Kurylov and M. J. Ramsey-Musolf, Phys. Rev. D 68 (2003) 016006

[143] P. E. Bosted et al., proposal at SLAC in preparation

[144] M. J. Ramsey-Musolf, Phys. Rev. C 60 (1999) 015501

[145] B. P. Masterson and C. E. Wieman, p. 545 of Ref. 866]

[146] T. P. Emmons, J. M. Reeves and E. N. Fortson, Phys. Rev. Lett. 51 (1983) 2089

[147] J. H. Hollister et al., Phys. Rev. Lett. 46 (1981) 642

[148] M. J. D. MacPherson et al., Phys. Rev. Lett. 67 (1991) 2784

[149] D. M. Meekhof et al., Phys. Rev. Lett. 71 (1993) 3442

[150] T. Wolfeden, P. Baird and P. Sandars, Eur. Phys. J. 15 (1991) 731

[151] P. S. Drell and E. D. Commins, Phys. Rev. Lett. 53 (1984) 968

[152] N. H. Edwards et al., Phys. Rev. Lett. 74 (1995) 2654

[153] P. A. Vetter et al., Phys. Rev. Lett. 74 (1995) 2658

[154] S. L. Gilbert, M. C. Noecker, R. N. Watts and C. E. Wieman, Phys. Rev. Lett. 55 (1985) 2680

[155] M. A. Bouchiat et al., J. Phys. (Paris) 47 (1986) 1709

[156] M. C. Noecker, B. P. Masterson and C. E. Wieman, Phys. Rev. Lett. 61 (1988) 310

[157] D. DeMille, Phys. Rev. Lett. 74 (1995) 4165

[158] D. F. Kimball, Phys. Rev. A 63 (2001) 052113

[159] J. Stalnaker, private communication

[160] N. Fortson, private communication 
[161] L. Orozco, private communication

[162] W. C. Haxton and C. E. Wieman, Ann. Rev. Nucl. Part. Sci. 51 (2001) 261

[163] S. J. Pollock and M. C. Welliver, Phys. Lett. B 464 (1999) 177

[164] S. A. Blundell, W. R. Johnson and J. Sapirstein, Phys. Rev. Lett. 65 (1990) 1411

[165] S. A. Blundell, J. R. Sapirstein and W. R. Johnson, Phys. Rev. D 45 (1992) 1602

[166] V. A. Dzuba, V. V. Flambaum and O. P. Sushkov, Phys. Lett. A 141 (1989) 147

[167] J. L. Rosner, Phys. Rev. D 61 (2000) 016006

[168] R. Casalbuoni et al., preprint hep-ph/0001215, talk given at 2nd ECFA/DESY Study 1998-2001

[169] V. D. Barger and K. M. Cheung, Phys. Lett. B 480 (2000) 149

[170] M. J. Ramsey-Musolf, Phys. Rev. D 62 (2000) 056009

[171] A. Derevianko, Phys. Rev. Lett. 85 (2000) 1618

[172] V. A. Dzuba, C. Harabati, W. R. Johnson, and M. S. Safronova, Phys. Rev. A 63 (2001) 044103

[173] W. R. Johnson, I. Bednyakov and G. Soff, Phys. Rev. Lett. 87 (2001) 233001

[174] A. I. Milstein, O. P. Sushkov and I. S. Terekhov, Phys. Rev. Lett. 89 (2002) 283003

[175] V. A. Dzuba, V. V. Flambaum and J. S. Ginges, Phys. Rev. D 66 (2002) 076013

[176] M. Y. Kuchiev and V. V. Flambaum, Phys. Rev. Lett. 89 (2002) 283002

[177] A. I. Milstein, O. P. Sushkov and I. S. Terekhov, preprint hep-ph/0212072

[178] M. Y. Kuchiev and V. V. Flambaum, preprint hep-ph/0305053.

[179] V. A. Dzuba, V. V. Flambaum and J. S. M. Ginges, Phys. Rev. A 63 (2001) 062101

[180] R. Michaels and P. Souder, spokespersons, proposal JLAB-PR-99-012,

http://www.jlab.org/exp_prog/proposals/00/PR99-012.pdf

[181] W. J. Marciano, Phys. Rev. D 60 (1999) 093006

[182] S. M. Berman, Phys. Rev. 112 (1958) 267

[183] T. Kinoshita and A. Sirlin, Phys. Rev. 113 (1959) 1652

[184] M. Roos and A. Sirlin, Nucl. Phys. B 29 (1971) 296

[185] T. van Ritbergen and R. G. Stuart, Phys. Rev. Lett. 82 (1999) 488

[186] A. Kurylov and M. J. Ramsey-Musolf, Phys. Rev. Lett. 88 (2002) 071804

[187] M. Drees, K. Hagiwara and A. Yamada, Phys. Rev. D 45 (1992) 1725

[188] G. Altarelli, R. Barbieri and F. Caravaglios, Phys. Lett. B 314 (1993) 357

[189] D. Garcia and J. Sola, Mod. Phys. Lett. A 9 (1994) 211

[190] P. H. Chankowski et al., Nucl. Phys. Proc. Suppl. B 37 (1994) 232

[191] G. C. Cho and K. Hagiwara, Nucl. Phys. B 574 (2000) 623

[192] W. de Boer et al., Z. Phys. C 71 (1996) 415

[193] D. M. Pierce, J. A. Bagger, K. T. Matchev and R. Zhang, Nucl. Phys. B 491 (1997) 3

[194] J. Erler and D. M. Pierce, Nucl. Phys. B 526 (1998) 53

[195] PSI-Experiment R99.06.1, Precision Measurement of the $\mu^{+}$Lifetime $\left(G_{F}\right)$ with the FAST Detector, J. Kirkby and M. Pohl, co-spokespersons

[196] PSI-Experiment R99.07.1, A Measurement of the Positive Muon Lifetime utilizing the $\mu$ Lan (MUON Lifetime ANalysis) Detector, D. Hertzog, contact person

[197] J. Aysto et al., preprint hep-ph/0109217

[198] L. Michel, Proc. Phys. Soc. A 63 (1950) 514 
[199] C. Bouchiat and L. Michel, Phys. Rev. 106 (1957) 170

[200] S. E. Derenzo, Phys. Rev. 181 (1969) 1854

[201] B. K. E. Balke, preprint UMI-87-26134

[202] I. Beltrami et al., Phys. Lett. B 194 (1987) 326

[203] H. Burkard et al., Phys. Lett. B 150 (1985) 242

[204] TWIST Collaboration: J. M. Poutissou et al., Nucl. Phys. A 721 (2003) 465

[205] E. D. Commins and P. H. Bucksbaum, Weak Interactions of Leptons and Quarks (Cambridge University Press, Cambridge, 1983)

[206] P. Herczeg, Phys. Rev. D 34 (1986) 3449

[207] W. J. Marciano and A. Sirlin, Phys. Rev. Lett. 71 (1993) 3629

[208] G. Czapek et al., Phys. Rev. Lett. 70 (17) 1993

[209] D. I. Britton et al., Phys. Rev. Lett. 68 (1992) 3000

[210] V. D. Barger, G. F. Giudice and T. Han, Phys. Rev. D 40 (1989) 2987

[211] A. Sirlin, Rev. Mod. Phys. 50 (1978) 573

[212] V. Cirigliano, M. Knecht, H. Neufeld and H. Pichl, Eur. Phys. J. C 27 (2003) 255

[213] W. K. McFarlane et al., Phys. Rev. D 32 (1985) 547

[214] PIBETA Collaboration: D. Pocanic et al., preprint hep-ex/0311013, talk presented at NAPP 2003

[215] D. Pocanic et al., preprint hep-ex/0312030

[216] A. A. Poblaguev, Phys. Lett. B 238 (1990) 108

[217] P. Herczeg, Phys. Rev. D 49 (1994) 247

[218] E. Frlez et al., preprint hep-ex/0312029

[219] J. D. Jackson, S. B. Treiman and H. W. Wyld, Phys. Rev. 106 (517) 1957

[220] emiT Collaboration: L. J. Lising et al., Phys. Rev. C 62 (2000) 055501

[221] P. R. Huffman et al., talk presented at International Workshop On Quark Mixing, CKM Unitarity

[222] H. Abele et al., Phys. Rev. Lett. 88 (2002) 211801

[223] A. Saunders et al., preprint nucl-ex/0312021

[224] S. Gardner and C. Zhang, Phys. Rev. Lett. 86 (2001) 5666

[225] S. Ando et al., Phys. Lett. B 595 (2004) 250

[226] I. S. Towner and J. C. Hardy, preprint nucl-th/9504015

[227] I. S. Towner and J. C. Hardy, preprint nucl-th/9809087, talk presented at WEIN 98

[228] J. C. Hardy and I. S. Towner, Eur. Phys. J. A 15 (2002) 223

[229] I. S. Towner and J. C. Hardy, Phys. Rev. C 66 (2002) 035501

[230] A. Sher et al., Phys. Rev. Lett. 91 (2003) 261802

[231] V. Cirigliano et al., Eur. Phys. J. C 23 (2002) 121

[232] H. Leutwyler and M. Roos, Z. Phys. C 25 (1984) 91

[233] J. Gasser and H. Leutwyler, Nucl. Phys. B 250 (1985) 517

[234] P. Post and K. Schilcher, Eur. Phys. J. C 25 (2002) 427

[235] J. Bijnens and P. Talavera, Nucl. Phys. B 669 (2003) 341

[236] KLOE Collaboration: M. Moulson et al., AIP Conf. Proc. 698 (2004) 74

[237] NA48/2 Collaboration: D. Madigozhin et al., eConf C0304052 (2003) WG605

[238] V. Cirigliano, H. Neufeld, and R. Kessler, private communication 
[239] A. Kurylov, M. J. Ramsey-Musolf and S. Su, Phys. Rev. D 68 (2003) 035008

[240] A. Kurylov, M. J. Ramsey-Musolf and S. Su, Phys. Lett. B 582 (2004) 222

[241] NA48 Collaboration: J. R. Batley et al., Phys. Lett. B 544 (2002) 97

[242] KTeV Collaboration: A. Alavi-Harati et al., Phys. Rev. D 67 (2003) 012005

[243] C. Jarlskog, Phys. Rev. Lett. 55 (1985) 1039

[244] N. Fortson, P. Sandars and S. Barr, Phys. Today 56N6 (2003) 33

[245] J. S. M. Ginges and V. V. Flambaum, preprint physics/0309054

[246] I. B. Khriplovich and S. K. Lamoreaux, CP Violation Without Strangeness: Electric Dipole Moments of Particles, Atoms, and Molecules (Springer, Berlin, 1997)

[247] V. F. Dmitriev and I. B. Khriplovich, Phys. Rep. 391 (2004) 243

[248] M. Gell-Mann, P. Ramond and R. Slansky, p. 315 in Supergravity, P. van Nieuwenhuizen and D. Z. Freedman (eds.), (North-Holland, Amsterdam, 1979)

[249] R. N. Mohapatra and G. Senjanovic, Phys. Rev. Lett. 44 (1980) 912

[250] T. Yanagida, Prog. Theor. Phys. 64 (1980) 1103

[251] B. C. Regan, E. D. Commins, C. J. Schmidt and D. DeMille, Phys. Rev. Lett. 88 (2002) 071805

[252] P. G. H. Sandars, Phys. Lett. 14 (1965) 194

[253] Z. W. Liu and H. P. Kelly, Phys. Rev. A 45 (1992) R4210

[254] D. DeMille et al., Phys. Rev. A 61 (2000) 052507

[255] D. Kawall et al., preprint hep-ex/0309079

[256] P. G. H. Sandars, Phys. Rev. Lett. 19 (1967) 1396

[257] O. P. Sushkov and V. V. Flambaum, Zh. Eksp. Teor. Fiz. 75 (1978) 1208

[258] M. G. Kozlov and L. N. Labzowsky, J. Phys. B 28 (1995) 1933

[259] M. G. Kozlov and D. DeMille, Phys. Rev. Lett. 89 (2002) 133001

[260] T. A. Isaev et al., preprint physics/0306071

[261] C. Y. Liu and S. Lamoreaux, private communication

[262] F. L. Shapiro, Usp. Fiz. Nauk. 95 (1968) 145

[263] EDM Collaboration: Y. K. Semertzidis et al., AIP Conf. Proc. 698 (2004) 200

[264] E. M. Purcell and N. F. Ramsey, Phys. Rev. 78 (1950) 807

[265] J. H. Smith, E. M. Purcell and N. F. Ramsey, Phys. Rev. 108 (1957) 120

[266] P. G. Harris et al., Phys. Rev. Lett. 82 (1999) 904

[267] P. G. Harris et al., Nucl. Instrum. Meth. A 440 (2000) 479

[268] EDM Collaboration: R. E. Mischke, AIP Conf. Proc. 675 (2003) 246

[269] E. Aleksandrov et al., http://ucn.web.psi.ch/papers/annual_report_02/R-00-05_mag_ru-main.pdf

[270] M. V. Romalis, W. C. Griffith and E. N. Fortson, Phys. Rev. Lett. 86 (2001) 2505

[271] M. V. Romalis and M. P. Ledbetter, Phys. Rev. Lett. 87 (2001) 067601

[272] M. V. Romalis, private communication

[273] R. Holt, private communication

[274] J. Behr, Nucl. Inst. Meth. B 204 (2003) 526

[275] E. P. Shabalin, Sov. J. Nucl. Phys. 28 (1978) 75 and Yad. Fiz. 31 (1980) 1665

[276] E. P. Shabalin, Sov. Phys. Usp. 26 (1983) 297

[277] W. Bernreuther and M. Suzuki, Rev. Mod. Phys. 63 (1991) 313 
[278] M. B. Gavela et al., Phys. Lett. B 109 (1982) 83 and 215

[279] I. B. Khriplovich and A. R. Zhitnitsky, Phys. Lett. B 109 (1982) 490

[280] X. G. He, B. H. J. McKellar and S. Pakvasa, Int. J. Mod. Phys. A 4 (1989) 5011

[281] W. C. Haxton and E. M. Henley, Phys. Rev. Lett. 51 (1983) 1937

[282] V. V. Flambaum, I. B. Khriplovich and O. P. Sushkov, J. Exp. Theor. Phys. 60 (1984) 873 and Nucl. Phys. A 449 (1986) 750

[283] J. F. Donoghue, B. R. Holstein and M. J. Musolf, Phys. Lett. B 196 (1987) 196

[284] L. I. Schiff, Phys. Rev. D 132 (1963) 2194

[285] J. Engel et al., Phys. Rev. C 68 (2003) 025501

[286] J. Engel, J. L. Friar and A. C. Hayes, Phys. Rev. C 61 (2000) 035502

[287] V. A. Dzuba, V. V. Flambaum, J. S. M. Ginges and M. G. Kozlov, Phys. Rev. A 66 (2002) 012111

[288] V. F. Dmitriev and R. A. Sen'kov, Phys. Rev. Lett. 91 (2003) 212303

[289] I. B. Khriplovich and R. A. Korkin, Nucl. Phys. A 665 (2000) 365

[290] R. J. Crewther, P. Di Vecchia, G. Veneziano and E. Witten, Phys. Lett. B 88 (1979) 123

[291] K. Kawarabayashi and N. Ohta, Nucl. Phys. B 175 (1980) 477 and Prog. Theor. Phys. 66 (1981) 1789

[292] T. Falk, K. A. Olive, M. Pospelov and R. Roiban, Nucl. Phys. B 560 (1999) 3

[293] R. S. Conti and I. B. Khriplovich, Phys. Rev. Lett. 68 (1992) 3262

[294] J. Engel, P. H. Frampton and R. P. Springer, Phys. Rev. D 53 (1996) 5112

[295] M. J. Ramsey-Musolf, Phys. Rev. Lett. 83 (1999) 3997

[296] A. Kurylov, G. C. McLaughlin and M. J. Ramsey-Musolf, Phys. Rev. D 63 (2001) 076007

[297] A. D. Sakharov, JETP Lett. 5 (1967) 24

[298] S. M. Barr and W. J. Marciano, Adv. Ser. Direct. High Energy Phys. 3 (1989) 455

[299] A. Brignole, J. R. Espinosa, M. Quiros and F. Zwirner, Phys. Lett. B 324 (1994) 181

[300] A. Riotto and M. Trodden, Ann. Rev. Nucl. Part. Sci. 49 (1999) 35

[301] P. Huet and A. E. Nelson, Phys. Rev. D 53 (1996) 4578

[302] A. Riotto, Phys. Rev. D 58 (1998) 095009

[303] MEGA Collaboration: M. L. Brooks et al., Phys. Rev. Lett. 83 (1999) 1521

[304] P. Wintz, talk presented at ICHEP 98

[305] SINDRUM Collaboration: U. Bellgardt et al., Nucl. Phys. B 299 (1988) 1

[306] SINDRUM II Collaboration: J. Kaulard et al., Phys. Lett. B 422 (1998) 334

[307] J. Yashima et al., in New Initiatives on Lepton Flavor Violation and Neutrino Oscillation With High Intense Muon and Neutrino Sources, 2-6 Oct 2000, Honolulu, HI, Y. Kuno et al. (eds.)

[308] W. Molzon, Nucl. Phys. Proc. Suppl. B 111 (2002) 188

[309] Riazuddin, R. E. Marshak and R. N. Mohapatra, Phys. Rev. D 24 (1981) 1310

[310] G. K. Leontaris, K. Tamvakis and J. D. Vergados, Phys. Lett. B 171 (1986) 412

[311] F. Borzumati and A. Masiero, Phys. Rev. Lett. 57 (1986) 961

[312] R. Barbieri and L. J. Hall, Phys. Lett. B 338 (1994) 212

[313] R. Barbieri, L. J. Hall and A. Strumia, Nucl. Phys. B 445 (1995) 219

[314] J. Hisano et al., Phys. Lett. B 357 (1995) 579

[315] G. Barenboim and M. Raidal, Nucl. Phys. B 484 (1997) 63 
[316] K. Huitu, J. Maalampi, M. Raidal and A. Santamaria, Phys. Lett. B 430 (1998) 355

[317] M. Raidal and A. Santamaria, Phys. Lett. B 421 (1998) 250

[318] V. Cirigliano, A. Kurylov, M. Ramsey-Musolf and P. Vogel, preprint hep-ph/0404223

[319] S. L. Glashow, J. Iliopoulos and L. Maiani, Phys. Rev. D 2 (1970) 1285

[320] R. D. McKeown and P. Vogel, Phys. Rep. 394 (2004) 315

[321] D. N. Spergel et al., Astrophys. J. Suppl. 148 (2003) 175

[322] E. Majorana, Nuovo Cimento 14 (1937) 171

[323] G. B. Gelmini and M. Roncadelli, Phys. Lett. B 99 (1981) 411

[324] P. Langacker, p. 863 in Testing The Standard Model, M. Cvetič and P. Langacker (eds.), (World Scientific, Singapore, 1991)

[325] Z. Maki, M. Nakagawa and S. Sakata, Prog. Theor. Phys. 28 (1962) 870

[326] R. Barbieri, J. R. Ellis and M. K. Gaillard, Phys. Lett. B 90 (1980) 249

[327] C. Weinheimer et al., Phys. Lett. B 460 (1999) 219

[328] V. M. Lobashev et al., Phys. Lett. B 460 (1999) 227

[329] P. Vogel and A. Piepke, p. 380 of Ref. [113]

[330] KATRIN Collaboration: A. Osipowicz et al., preprint hep-ex/0109033

[331] S. M. Bilenky, C. Giunti, J. A. Grifols and E. Masso, Phys. Rep. 379 (2003) 69

[332] B. Pontecorvo, J. Exp. Theor. Phys. 6 (1957) 429

[333] V. N. Gribov and B. Pontecorvo, Phys. Lett. B 28 (1969) 493

[334] S. M. Bilenky and B. Pontecorvo, Phys. Rep. 41 (1978) 225

[335] B. Kayser, Phys. Rev. D 24 (1981) 110

[336] J. D. Vergados, Phys. Rep. 133 (1986) 1

[337] S. M. Bilenky and S. T. Petcov, Rev. Mod. Phys. 59 (1987) 671

[338] S. M. Bilenky, C. Giunti and W. Grimus, Prog. Part. Nucl. Phys. 43 (1999) 1

[339] S. M. Bilenky and B. Pontecorvo, Lett. Nuovo Cimento 17 (1976) 569

[340] H. Murayama, p. 401 of Ref. 113

[341] K. Nakamura, p. 408 of Ref. [113]

[342] S. Goswami, preprint hep-ph/0303075

[343] J. N. Bahcall, M. C. Gonzalez-Garcia and C. Peña-Garay, JHEP 0204 (2002) 007

[344] J. N. Bahcall, M. H. Pinsonneault and S. Basu, Astrophys. J. 555 (2001) 990

[345] A. R. Junghans et al., Phys. Rev. Lett. 88 (2002) 041101

[346] E. G. Adelberger et al., Rev. Mod. Phys. 70 (1998) 1265

[347] ISOLDE Collaboration: L. T. Baby et al., Phys. Rev. Lett. 90 (2003) 022501

[348] B. T. Cleveland et al., Astrophys. J. 496 (1998) 505

[349] SAGE Collaboration: J. N. Abdurashitov et al., J. Exp. Theor. Phys. 95 (2002) 181

[350] GALLEX Collaboration: W. Hampel et al., JournalPhys. Lett. B4471271999

[351] GNO Collaboration: M. Altmann et al., Phys. Lett. B 490 (2000) 16

[352] Kamiokande Collaboration: Y. Fukuda et al., Phys. Rev. Lett. 77 (1996) 1683

[353] Super-Kamiokande Collaboration: S. Fukuda et al., Phys. Lett. B 539 (2002) 179

[354] Super-Kamiokande Col.: M. B. Smy et al., preprint hep-ex/0208040, talk given at Neutrino 2002 [355] SNO Collaboration: Q. R. Ahmad et al., Phys. Rev. Lett. 89 (2002) 011301 
[356] Super-Kamiokande Collaboration: Y. Gando et al., Phys. Rev. Lett. 90 (2003) 171302

[357] S. Bludman, N. Hata and P. Langacker, Phys. Rev. D 49 (1994) 3622

[358] G. L. Fogli, E. Lisi and D. Montanino, Phys. Rev. D 49 (1994) 3626

[359] E. K. Akhmedov, A. Lanza and S. T. Petcov, Phys. Lett. B 348 (1995) 124

[360] V. Berezinsky, G. Fiorentini and M. Lissia, Phys. Lett. B 365 (1996) 185

[361] P. I. Krastev and S. T. Petcov, Nucl. Phys. B 449 (1995) 605

[362] Borexino Collaboration: G. Alimonti et al., Astropart. Phys. 16 (2002) 205

[363] SNO Collaboration: Q. R. Ahmad et al., Phys. Rev. Lett. 89 (2002) 011302

[364] S. P. Mikheev and A. Y. Smirnov, Sov. J. Nucl. Phys. 42 (1985) 913

[365] L. Wolfenstein, Phys. Rev. D 17 (1978) 2369

[366] V. Barger and D. Marfatia, Phys. Lett. B 555 (2003) 144

[367] G. L. Fogli et al., Phys. Rev. D 67 (2003) 073002

[368] M. Maltoni, T. Schwetz and J. W. Valle, Phys. Rev. D 67 (2003) 093003

[369] A. Bandyopadhyay et al., Phys. Lett. B 559 (2003) 121

[370] H. Nunokawa, W. J. Teves and R. Zukanovich Funchal, Phys. Lett. B 562 (2003) 28

[371] P. Aliani, V. Antonelli, M. Picariello and E. Torrente-Lujan, Phys. Rev. D 69 (2004) 013005

[372] P. C. de Holanda and A. Y. Smirnov, JCAP 0302 (2003) 001

[373] A. B. Balantekin and H. Yuksel, J. Phys. G 29 (2003) 665

[374] J. N. Bahcall and C. Peña-Garay, JHEP 0311 (2003) 004

[375] SNO Collaboration: S. N. Ahmed et al., preprint nucl-ex/0309004

[376] Kamiokande-II Collaboration: K. S. Hirata et al., Phys. Lett. B 280 (1992) 146

[377] Kamiokande Collaboration: Y. Fukuda et al., Phys. Lett. B 335 (1994) 237

[378] R. Becker-Szendy et al., Phys. Rev. D 46 (1992) 3720

[379] Frejus Collaboration: K. Daum et al., Z. Phys. C 66 (1995) 417

[380] Super-Kamiokande Collaboration: Y. Fukuda et al., Phys. Lett. B 433 (1998) 9

[381] Super-Kamiokande Collaboration: Y. Fukuda et al., Phys. Lett. B 436 (1998) 33

[382] Soudan-2 Collaboration: W. W. Allison et al., Phys. Lett. B 449 (1999) 137

[383] Super-Kamiokande Collaboration: Y. Fukuda et al., Phys. Rev. Lett. 81 (1998) 1562

[384] Super-Kamiokande Collaboration: Y. Fukuda et al., Phys. Rev. Lett. 82 (1999) 2644

[385] Super-Kamiokande Collaboration: S. Fukuda et al., Phys. Lett. B 467 (1999) 185

[386] Super-Kamiokande Collaboration: S. Fukuda et al., Phys. Rev. Lett. 85 (2000) 3999

[387] MACRO Collaboration: M. Ambrosio et al., Phys. Lett. B 517 (2001) 59

[388] MACRO Collaboration: M. Ambrosio et al., Phys. Lett. B 566 (2003) 35

[389] G. L. Fogli, E. Lisi, A. Marrone and D. Montanino, Phys. Rev. D 67 (2003) 093006

[390] D. E. Groom, p. 399 of Ref. [113]

[391] KARMEN Collaboration: B. Armbruster et al., Phys. Rev. D 65 (2002) 112001

[392] LSND Collaboration: A. Aguilar et al., Phys. Rev. D 64 (2001) 112007

[393] BooNE Collaboration: E. Church et al., preprint nucl-ex/9706011

[394] G. Barenboim, L. Borissov, J. Lykken and A. Y. Smirnov, JHEP 0210 (2002) 001

[395] V. Barger et al., Phys. Lett. B 566 (2003) 8

[396] CHORUS Collaboration: E. Eskut et al., Phys. Lett. B 497 (2001) 8 
[397] NOMAD Collaboration: P. Astier et al.,Nucl. Phys. B 611 (2001) 3

[398] CERN-PS-169 Collaboration: F. Dydak et al., Phys. Lett. B 134 (1984) 281

[399] K2K Collaboration: M. H. Ahn et al., Phys. Rev. Lett. 90 (2003) 041801

[400] MINOS Collaboration: R. Saakian et al., Nucl. Phys. Proc. Suppl. B 111 (2002) 169

[401] OPERA and ICARUS Collns.: D. Duchesneau et al., Nucl. Phys. Proc. Suppl. 123 (2003) 279

[402] Y. Declais et al., Nucl. Phys. B 434 (1995) 503

[403] M. Apollonio et al., Eur. Phys. J. C 27 (2003) 331

[404] F. Boehm et al., Phys. Rev. D 64 (2001) 112001

[405] KamLAND Collaboration: K. Eguchi et al., Phys. Rev. Lett. 90 (2003) 021802

[406] S. R. Elliott and P. Vogel, Ann. Rev. Nucl. Part. Sci. 52 (2002) 115

[407] L. Baudis et al., Phys. Rev. Lett. 83 (1999) 41

[408] H. V. Klapdor-Kleingrothaus et al., Eur. Phys. J. A 12 (2001) 147

[409] IGEX Collaboration: C. E. Aalseth et al., Phys. Rev. C 59 (1999) 2108

[410] C. E. Aalseth et al., Phys. Atom. Nucl. 63 (2000) 1225

[411] R. Luscher et al., Phys. Lett. B 434 (1998) 407

[412] A. Alessandrello et al., Phys. Lett. B 486 (13) 2000

[413] H. Ejiri et al., Phys. Rev. C 63 (2001) 065501

[414] H. V. Klapdor-Kleingrothaus, preprint hep-ph/0103074 talk presented at NOON 2000

[415] Majorana Collaboration: C. E. Aalseth et al., preprint hep-ex/0201021, talk given at NANP 01

[416] F. A. Danevich et al., Phys. Rev. C 62 (2000) 045501

[417] C. Arnaboldi et al., AIP Conf. Proc. 605 (2002) 469

[418] H. Ejiri et al., Phys. Rev. Lett. 85 (2000) 2917

[419] H. V. Klapdor-Kleingrothaus et al., Mod. Phys. Lett. A 16 (2001) 2409

[420] C. E. Aalseth et al., Mod. Phys. Lett. A 17 (2002) 1475

[421] H. V. Klapdor-Kleingrothaus et al., preprint hep-ph/0404062

[422] A. Faessler, S. Kovalenko, F. Simkovic and J. Schwieger, Phys. Rev. Lett. 78 (1997) 183

[423] H. Paes, M. Hirsch, H. V. Klapdor-Kleingrothaus, S. G. Kovalenko, Phys. Lett. B 498 (2001) 35

[424] G. Prézeau, M. Ramsey-Musolf and P. Vogel, Phys. Rev. D 68 (2003) 034016

[425] T. Kinoshita and W. J. Marciano, p. 419 of Quantum Electrodynamics, T. Kinoshita (ed.), Advanced series on directions in high energy physics: 7 (World Scientific, Singapore, 1990)

[426] Muon g-2 Collaboration: G. W. Bennett et al., preprint hep-ex/0401008

[427] A. Czarnecki and W. J. Marciano, Phys. Rev. D 64 (2001) 013014

[428] J. L. Lopez, D. V. Nanopoulos, and X. Wang, Phys. Rev. D 49 (1994) 366

[429] J. Erler and M. Luo, Phys. Rev. Lett. 87 (2001) 071804

[430] M. Gourdin and E. De Rafael, Nucl. Phys. B 10 (1969) 667

[431] M. Davier, S. Eidelman, A. Höcker and Z. Zhang, Eur. Phys. J. C 31 (2003) 503

[432] CMD-2 Collaboration: R. R. Akhmetshin et al., Phys. Lett. B 527 (2002) 161

[433] K. Hagiwara, A. D. Martin, D. Nomura and T. Teubner, preprint hep-ph/0312250

[434] S. Ghozzi and F. Jegerlehner, Phys. Lett. B 583 (2004) 222

[435] F. Matorras, talk presented at EPS 2003

[436] S. Binner, J. H. Kühn and K. Melnikov, Phys. Lett. B 459 (1999) 279 
[437] V. Cirigliano, G. Ecker and H. Neufeld, JHEP 0208 (2002) 002

[438] W. J. Marciano and A. Sirlin, Phys. Rev. Lett. 61 (1988) 1815

[439] J. Erler, preprint hep-ph/0211345

[440] M. Knecht and A. Nyffeler, Phys. Rev. D 65 (2002) 073034

[441] M. Hayakawa and T. Kinoshita, preprint hep-ph/0112102

[442] J. Bijnens, E. Pallante and J. Prades, Nucl. Phys. B 626 (2002) 410

[443] M. Ramsey-Musolf and M. B. Wise, Phys. Rev. Lett. 89 (2002) 041601

[444] B. Krause, Phys. Lett. B 390 (1997) 392

[445] T. Kinoshita and M. Nio, preprint hep-ph/0402206

[446] K. Melnikov and A. Vainshtein, preprint hep-ph/0312226

[447] J. F. Donoghue et al., Cambridge Monogr. Part. Phys. Nucl. Phys. Cosmol. 2 (1992) 1

[448] B. Borasoy and B. R. Holstein, Eur. Phys. J. C 6 (85) 1999

[449] J. M. Potter et al., Phys. Rev. Lett. 33 (1974) 1307

[450] D. E. Nagle et al., AIP Conf. Proc. 51 (1978) 224

[451] R. Balzer et al., Phys. Rev. C 30 (1984) 1409

[452] S. Kistryn et al., Phys. Rev. Lett. 58 (1987) 1616

[453] P. D. Eversheim et al., Phys. Lett. B 256 (1991) 11

[454] TRIUMF-E497-Collaboration: A. R. Berdoz et al., Phys. Rev. Lett. 87 (2001) 272301

[455] TRIUMF-E497-Collaboration: A. R. Berdoz et al., Phys. Rev. C 68 (2003) 034004

[456] J. Lang et al., Phys. Rev. Lett. 54 (1985) 170

[457] J. Lang et al., Phys. Rev. C 34 (1986) 1545

[458] V. P. Alfimenkov et al., Nucl. Phys. A 398 (1983) 93

[459] Y. Masuda et al., Nucl. Phys. A 504 (1989) 269

[460] V. W. Yuan et al., Phys. Rev. C 44 (1991) 2187

[461] E. G. Adelberger et al., Phys. Rev. C 27 (1983) 2833

[462] K. Elsener et al., Phys. Rev. Lett. 52 (1984) 1476

[463] K. Elsener et al., Nucl. Phys. A 461 (1987) 579

[464] K A. Snover et al., Phys. Rev. Lett. 41 (1978) 145

[465] E. D. Earle et al., Nucl. Phys. A 396 (1983) 221C

[466] C. A. Barnes et al., Phys. Rev. Lett. 40 (1978) 840

[467] M. Bini et al., Phys. Rev. Lett. 55 (1985) 795

[468] G. Ahrens et al., Nucl. Phys. A 390 (1982) 496

[469] S. A. Page et al., Phys. Rev. C 35 (1987) 1119

[470] W. C. Haxton, C. P. Liu and M. J. Ramsey-Musolf, Phys. Rev. Lett. 86 (2001) 5247

[471] W. S. Wilburn and J. D. Bowman, Phys. Rev. C 57 (1998) 3425

[472] NPDGamma Collaboration: G. S. Mitchell et al., Nucl. Inst. Meth. A 521 (2004) 468

[473] B. Desplanques, J. F. Donoghue and B. R. Holstein, Ann. Phys. (N. Y.) 124 (1980) 449

[474] V. M. Dubovik and S. V. Zenkin, Ann. Phys. (N. Y.) 172 (1986) 100

[475] G. B. Feldman, G. A. Crawford, J. Dubach and B. R. Holstein, Phys. Rev. C 43 (1991) 863

[476] B. R. Holstein, AIP Conf. Proc. 698 (2004) 176

[477] B. R. Holstein, talk presented at FB 17 
[478] B. R. Holstein et al., to be published

[479] D. B. Kaplan and A. Manohar, Nucl. Phys. B 310 (1988) 527

[480] M. J. Ramsey-Musolf, preprint nucl-th/0302049, talk presented at PAVI 2002

[481] D. H. Beck and B. R. Holstein, INTE 10 (2001) 1

[482] D. H. Beck and R. D. McKeown, Ann. Rev. Nucl. Part. Sci. 51 (2001) 189

[483] R. D. McKeown and M. J. Ramsey-Musolf, Mod. Phys. Lett. A 18 (2003) 75

[484] SAMPLE Collaboration: D. T. Spayde et al., Phys. Lett. B 583 (2004) 79

[485] SAMPLE Collaboration]: T. M. Ito et al., Phys. Rev. Lett. 92 (2004) 102003

[486] HAPPEX Collaboration: K. A. Aniol et al., preprint nucl-ex/0402004

[487] HAPPEX Collaboration, G. Cates, K. Kumar and D. Lhuillier, spokespersons, proposal JLAB-E-99-115, http://www.jlab.org/exp_prog/proposals/99/PR99-115.pdf

[488] K. Kumar, private communication

[489] HAPPEX Collaboration, D. Armstrong and R. Michaels, spokespersons, proposal JLAB-E-00-114, http://www.jlab.org/exp_prog/proposals/00/PR00-114.pdf

[490] A4 Collaboration: F. E. Maas et al., preprint nucl-ex/0401019.

[491] GØ Collaboration: D. Beck, spokesperson, proposal JLAB-E-01-116, http://www.jlab.org/exp_prog/proposals/01/PR01-116.pdf

[492] M. Pitt, private communication

[493] G. T. Garvey, W. C. Louis and D. H. White, Phys. Rev. C 48 (1993) 761

[494] FINeSSE Collaboration: L. Bugel et al., preprint hep-ex/0402007, proposal at Fermilab [495] W. M. Alberico et al., Nucl. Phys. A 651 (1999) 277

[496] S. F. Pate, Phys. Rev. Lett. 92 (2004) 082002 